\title{
Study of a New 5-DOF Parallel Mechanism for Multi- Directional 3D Printing
}

\section{Yesong Wang}

University of Science and Technology Beijing

\section{Changhuai Lyu}

University of Science and Technology Beijing

Jiang Liu ( $\sim$ liulaoshiteam@126.com )

University of Science and Technology Beijing

Jinguang Zhang

University of Science and Technology Beijing

Zhixin Jia

University of Science and Technology Beijing

\section{Original Article}

Keywords: Screw theory, Singularity, Inverse position solution, Monte Carlo

Posted Date: December 10th, 2020

DOI: https://doi.org/10.21203/rs.3.rs-122415/v1

License: (c) (i) This work is licensed under a Creative Commons Attribution 4.0 International License.

Read Full License 


\title{
Study of a New 5-DOF Parallel Mechanism for Multi-directional 3D Printing
}

\author{
Yesong Wang, Changhuai Lyu, Jiang Liu*, Jinguang Zhang and Zhixin Jia
}

\begin{abstract}
This paper first designs a new 5-DOF parallel mechanism with 5PUS-UPU for multi-directional 3D printing, and then analyses its DOF by traditional Grubler-Kutzbach and motion spiral theory. It theoretically shows that the mechanism meets the requirement of 5 dimensions of freedoms including three-dimensional movement and two-dimensional rotation. Basing on this, the real mechanism is built, but unfortunately it is found unstable in some positions. Grassmann line geometry method is applied to analyze its unstable problem caused by singular posture, and then an improving method is put forward to solve it. With the improved mechanism, closed loop vector method is employed to establish the inverse position equation of the parallel mechanism, and kinematics analysis is carried out to get the mapping relationships between position, speed and acceleration of moving and fixed platform, Monte Carlo method is used to analyze the workspace of the mechanism, to explore the influencing factors of workspace, and then to get the better workspace. Finally an experiment is designed to verify the mechanism working performance to satisfy the spatial motion requirements of multi-directional $3 \mathrm{D}$ printing.
\end{abstract}

Keywords: Screw theory; Singularity; Inverse position solution; Monte Carlo

\section{Introduction}

Parallel mechanism (PM) can be defined as a closedloop mechanism in which the moving platform and the fixed platform are connected by at least two independent kinematic chains. The mechanism has two or more degrees of freedom, and is driven in parallel. Common parallel mechanisms are Delta and Stewart. Unlike traditional series structure, parallel structure is widely used in automobile, aviation, medical treatment, education and other fields due to its small cumu-

\footnotetext{
${ }^{*}$ Correspondence: liulaoshiteam@126.com
}

School of Mechanial Engineering, University of Science and Technology

Beijing, Beijing, China

Full list of author information is available at the end of the article lative error, high precision, light weight of moving part, high speed, fast dynamic response, compact structure, high rigidity and large bearing capacity [1].

3D printing, also known as additive manufacturing, processes parts by adding materials layer by layer. Unlike traditional material removal technology, 3D printing process does not require any dies and machining, and generates various shapes through simple trajectory planning, which greatly improves production efficiency and reduces production costs $[2,3]$. The 3D printer based on parallel mechanism inherits the advantages of high rigidity-mass ratio, no accumulation of position errors and great loading ca- 
pacity of the parallel mechanism, and becomes a hot spot in robot research and application in the world [4-8]. At present, many scholars have applied parallel mechanisms with different postures to 3D printers and achieved many results. Among them, Delta mechanism has been successfully applied in cases of 3D printing technology based on three-dimensional translation and high accuracy [9]. Guo Xiaobo [10] and others analyzed the workspace of 3-HSS parallel 3D printer and Bi Changfei [11] did kinematics analysis and Simulation of 3-HSS parallel 3D printer. These 3D printers based on parallel mechanism have higher precision and better motion performance than those based on series mechanism, but for these 3D printers based on a 3-freedom parallel mechanism, their printing heads can only maintain one direction. However, during the printing process, it was found that they cannot print parts with complex surfaces or slopes with high quality.

Therefore, with the development of 3D printers, a concept of multi-directional printing has been proposed. This printing method can accumulate materials in different directions on the formed surface, which improves the quality of the surface of complex printed parts, but requires the mechanism with 5 degrees of freedom. Keating $\mathrm{S}$ et al. [12] designed a five-degree-offreedom 3D printer to achieve multi-directional printing. However, this robot adopts a serial mechanism, and the inertia and error of the entire mechanism are large. At present, there are relatively few studies on multi-directional 3D printing based on parallel mechanisms. Song et al. [13] applied the Stewart parallel mechanism to a 3D printer, but the six-degree-offreedom Stewart mechanism achieves a relatively small working space and has one redundant degree of freedom to rotate around the print head. Fang Yuefa et al. [14] designed a four-degree-of-freedom parallel multidirectional 3D printer and performed kinematic analy- sis. Wei Ye et al. [15] designed a reconfigurable parallel mechanism for multidirectional additive manufacturing, which has four motion modes to choose from, but can only achieve up to four degrees of freedom in one mode. Pan Ying et al. [16] designed a five-degree-offreedom parallel 3D printer, but with a compound dynamic platform, the complex structure is not suitable for promotion.

With the development of parallel mechanism, the analysis of singularity and optimization of workspace of parallel mechanism have become a hot research topic. J. Wang [17] used a $4 \times 4$ Jacobian matrix to analyze the singularity of a spatial 4-DOF parallel mechanism; J. Gallardo-Alvarado et al. [18] analyzed the singular configuration of a 4-DOF parallel robot by using the screw theory; Sheng Guo et al. [19] analyzed the singularity of a 4-RRCR parallel mechanism using srew theory; Thomas, M.J. [20] used a numerical based algorithm to determine the singular points within the workspace of the 3-PRUS mechanism by determining points where the determinant of the Jacobian becomes zero; Wolf [21] analyzed the singularity of two kinds of 3-DOF parallel robots by using the Grassmann line geometry method; Chunxu Tian et al. [22] analyzed singularities of a partially decoupled generalized parallel mechanism for 3T1R motion; Zongjie Tao et al. [23] deveoped system design methodology (LIDeM) for reducing interference among links and increasing workspace of mechanism; Liping Wang et al. [24] optimized 3-PUU parallel mechanism modules for different application objectives.

In this paper, a 5-DOF parallel mechanism based on multi-directional 3D printer is designed. The mechanism adopts parallel structure completely and the printer head is installed on the moving platform of the parallel mechanism. During the printing process, as the posture of the moving platform changes, the print head can realize multi-directional printing. As 
the driving part of the parallel mechanism, two methods of constant-length link drive and telescopic link drive are currently adopted. Comparing the two methods, the fixed-link length driving mode can greatly reduce the structural size of the link, which is not easy to produce interference in the process of movement [25]. Therefore, this driving mode is used to build parallel mechanism in this paper. Finally, we study the 5PUS-UPU five-degree-of-freedom parallel mechanism from aspects of scheme design, freedom degree verification, test equipment construction, singularity analysis, scheme improvement, mechanism motion analysis, workspace analysis, and experimental verification.

\section{Design and verification of the scheme}

\subsection{Design of the primary scheme}

At present, 6-DOF Stewart platform [26] is used as the prototype for 5-DOF parallel mechanism. The minimum degrees of freedom of the 3D printer structure that meets multi-directional printing is $5-3$ movements and 2 rotations. In the case of satisfying the degree of freedom requirement, the less degree of freedom of the mechanism, the simpler mechanical structure, the lower manufacturing and control costs. Therefore, we choose to remove one of the six active limbs in the Stewart platform, and then rearrange the spatial positions of the five active limbs. However, at this time, there are only 5 driving modules, while the number of degrees of freedom of the moving platform is 6 . The number of driving modules is less than the degrees of freedom of the mechanism, which will cause the motion of the moving platform to be uncertain. The redundant degree of freedom of the moving platform is limited by adding a restraint limb between the moving platform and the fixed platform. At this time, the number of degrees of freedom of the moving platform is 5 , which meets the requirement of the degree of freedom.

As for the drive part of parallel mechanism, fixedlength link drive and telescopic link drive are the two most widely used ways at present. The driving mode of the mobile pair with a fixed-length link can greatly reduce the structural dimension of the link and avoid interference during the movement [24]. Therefore, we choose the driving method of the mobile pair with a fixed-length link in this paper. The five mobile pairs are used as actuators, which are symmetrically distributed in the shape of regular pentagons in space. The moving platform also adopts the symmetrical structure of the positive pentagon. As shown in Figure 1, the mechanism consists of five PUS limbs and one UPU limb. Five PUS limbs are called driving limbs, where $P_{1} \sim P_{5}$ are used as driving elements to represent the mobile pairs and are distributed on the corners of the positive pentagon, which called sliders. $U_{1} \sim U_{5}$ represent Hook joints, connecting 5 sliders and 5 connecting links. $S_{1} \sim S_{5}$ represent the spherical hinges, connecting the moving platform and 5 connecting links. At the same time, the UPU limb is called the constrained limb, $U_{6}$ and $U_{6}^{\prime}$ represent Hook joints, which are fixed to the center of the regular pentagon and the center of the moving platform, respectively. $P_{6}$ represents the mobile pair, connecting Hook joints $U_{6}$ and $U_{6}^{\prime}$.

\subsection{Verification of the primary scheme}

\subsubsection{Verification of degree of freedom by Grubler-Kutzbach formula}

The number of degrees of freedom determines whether the designed mechanism meets the requirements, so the analysis of the degrees of freedom is particularly important. The traditional Grubler-Kutzbach method is relatively simple, only depends on the number of components and kinematic pairs of the mechanism, and the degree of freedom of the mechanism can be obtained only through the most basic mathematical calculations. Therefore, this paper firstly uses GrublerKutzbach method to verify the degree of freedom of the 


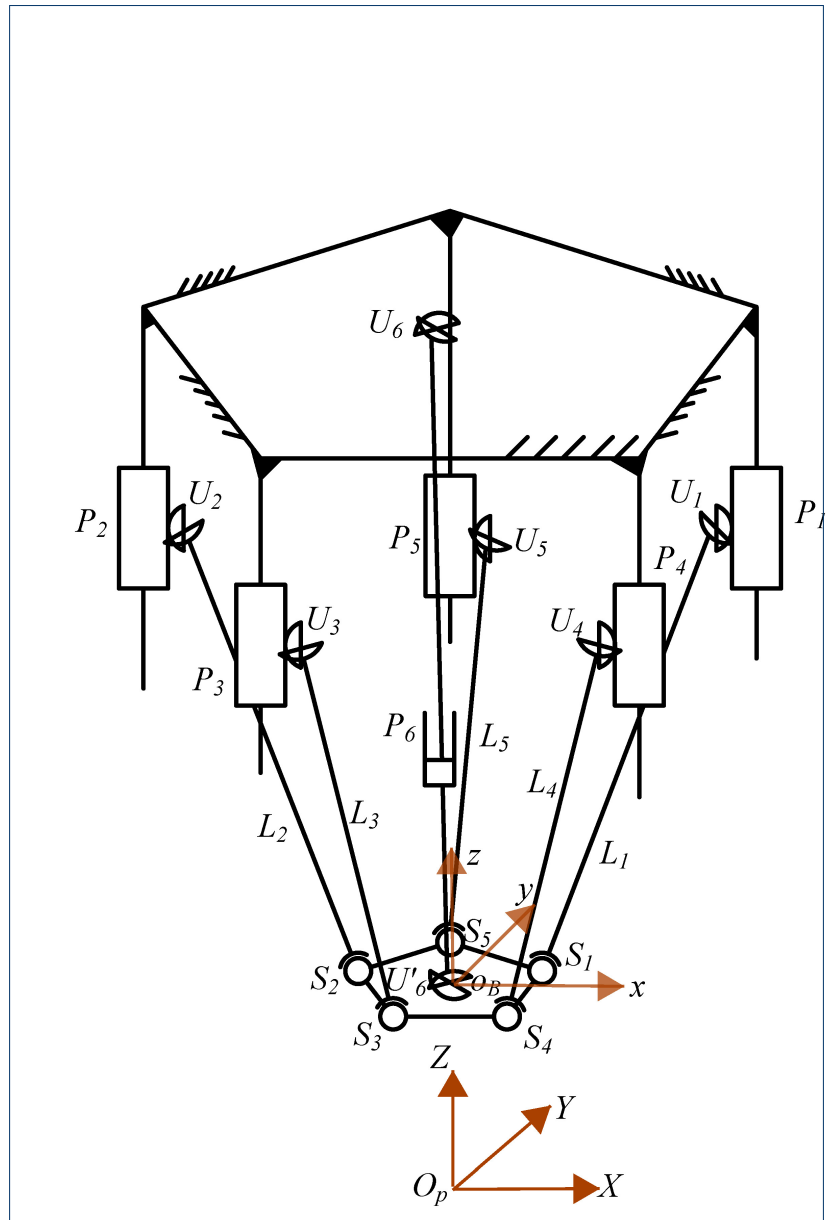

Figure 1 Design sketch of parallel mechanism

mechanism. The main contents of Grubler-Kutzbach method are as follows:

In three-dimensional space, when a component is free before it is connected with other components by kinematic pairs. The $n$ components including the frame have $6(n-1)$ degrees of freedom. When one component is connected to another component with a kinematic pair, the component is constrained to a certain extent and its degree of freedom is reduced. When the two components are connected by a kinematic pair with $f_{i}$ degrees of freedom, the degree of freedom of the original system is reduced by $6-f_{i}$ due to increased constraints. When the number of kinematic pairs con- tinues to increase to $g$, the degree of freedom of the system is reduced by

$$
6 g-\sum_{i=1}^{g} f_{i}
$$

At this time, the degree of freedom of the mechanism is

$$
M=6(n-g-1)+\sum_{i=1}^{g} f_{i}
$$

Where $M$ is the degree of freedom of the mechanism (Mobility); $n$ is the total number of components of the mechanism; $g$ is the number of kinematic pairs of the mechanism; $f_{i}$ is the number of degrees of freedom of the $i$ th kinematic pair.

In this mechanism, $n=14, g=18, \sum_{i=1}^{g} f_{i}=35$, and without virtual constraints, compound hinges, local degrees of freedom and other special circumstances, so

$$
M=6(n-g-1)+\sum_{i=1}^{g} f_{i}=6 \times(14-18-1)+35=5
$$

That means the mechanism has 5 degrees of freedom, and the five sliders are used as the driving part, and the mechanism can achieve a certain movement.

\subsubsection{Verification of degree of freedom by screw theory}

Traditional Grubler-Kutzbach method can only be calculated as 5 degrees of freedom of the mechanism, which is not clear about the specific situation of the degree of freedom. Therefore, after a long-term development, the idea of solving the degree of freedom of parallel mechanism by screw theory emerged. The degree of freedom of 5-PUS-UPU parallel mechanism is further analyzed by using screw theory. In screw theory, the screw is generally expressed as $\left(S ; S_{0}\right)$ or 
plücker coordinates $(a b c ; l m n)$ [27]. $S$ is the original part of the rotation and $S_{0}$ is the dual part of the rotation [16]. The reciprocal product of two screws $\left(\$_{1}=\left(S_{1} ; S_{1}^{0}\right), \$_{2}=\left(S_{2} ; S_{2}^{0}\right)\right)$ is defined as the sum of the dot products after the exchange of the original and dual part

$$
\$_{1} \circ \$_{2}=S_{1} \cdot S_{2}^{0}+S_{2} \cdot S_{1}^{0}
$$

When the reciprocal product formed by $\$_{1}$ and $\$_{2}$ is 0 :

$$
\$_{1} \circ \$_{2}=0
$$

They are reciprocal, and the screw $\$_{2}$ is called the reciprocal screw of $\$_{1}$. Therefore, the reciprocal screw can be obtained based on the fact that the reciprocal product of the two screws is 0 .

The parallel mechanism designed in this paper uses 5 identical PUS limbs as the active limbs and 1 UPU limb as the constrained limb. Now the centre point of the Hook joint is set as the origin $o$, the directions of the two axes are the $x$-axis and the $y$-axis, respectively, and the $z$-axis is perpendicular to the xoy plane to establish a coordinate system, as shown in Figure 2.

The kinematic pairs on the active limbs are sequentially decomposed into single-degree-of-freedom motions, and the achievable motion of active limb 1 is expressed in a screw form [28]:

$$
\begin{aligned}
& \$_{1}=\left(\begin{array}{llllll}
0 & 0 & 0 & l_{1} & m_{1} & n_{1}
\end{array}\right)
\end{aligned}
$$

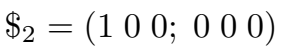

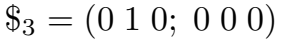

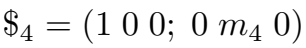

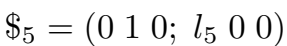

$$
\begin{aligned}
& \$_{6}=\left(\begin{array}{llllll}
0 & 0 & 1 ; & 0 & 0 & 0
\end{array}\right)
\end{aligned}
$$

Where $l_{1}, m_{1}, n_{1}, m_{4}, l_{5}$ represent coordinate parameters that are not zero, and $\$_{1}$ is the screw repre- sentation of moving pair $P_{1} ; \$_{2}$ is the spiral representation of degree of freedom of rotation of Hook joint $U_{1}$ around $x$-axis; $\$_{3}$ is the screw representation of degree of freedom of rotation of Hook joint $U_{1}$ around $y$-axis; $\$_{4}$ is the screw representation of degree of freedom of rotation of spherical hinge $S_{1}$ around $x$-axis; $\$_{5}$ is the screw representation of the degree of rotation of spherical hinge $S_{1}$ about the $y$-axis, and $\$_{6}$ is the spiral representation of the degree of rotation of spherical hinge $S_{1}$ about the $z$-axis.

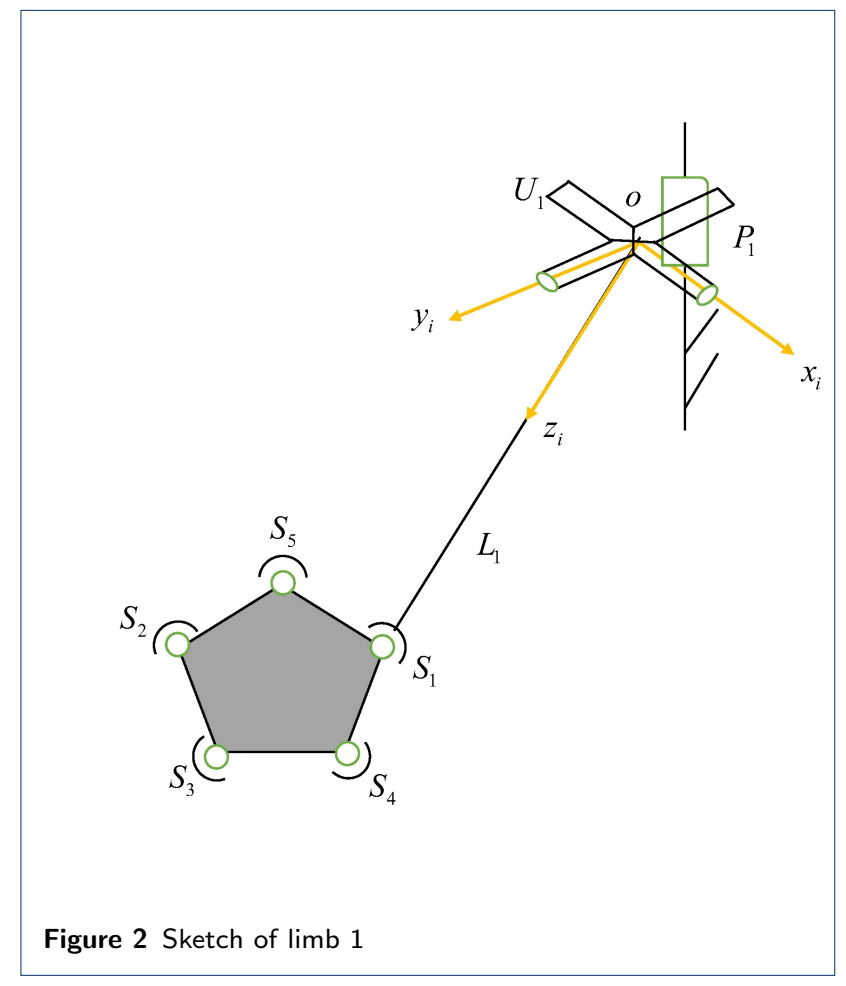

The screw system consisting of $\$_{1}, \$_{2}, \$_{3}, \$_{4}, \$_{5}$, and $\$ 6$ has been simplified to:

$$
\left(\begin{array}{ccc:ccc}
1 & 0 & 0 & 0 & 0 & 0 \\
0 & 1 & 0 & 0 & 0 & 0 \\
0 & 0 & 1 & 0 & 0 & 0 \\
0 & 0 & 0 & l_{5} & 0 & 0 \\
0 & 0 & 0 & 0 & m_{4} & 0 \\
0 & 0 & 0 & 0 & 0 & n_{1}
\end{array}\right)
$$


The twist of the active limb has a full rank, so there is no reciprocal screw. Similarly, the other four active limbs have the same analysis results. It can be seen that the five active limbs have no restriction on the moving platform [29].

When analyzing the constraint of the constraint limb to the moving platform, constrained limb coordinate system $o_{6}-x_{6} y_{6} z_{6}$ is established. Similarly, the centre point of Hook joint is set as origin $o$, and the two axes of Hook joint are set as $x_{6}$-axes and $y_{6}$-axes respectively, and the $z_{6}$ axes are perpendicular to the plane of $x_{6} O_{6} y_{6}$ to establish coordinate system, as shown in Figure 3.

Then, the screw system of the restrained limb is represented as:

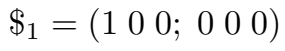

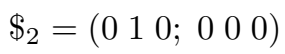

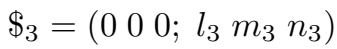

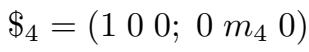

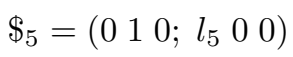

Where $l_{3}, m_{3}, n_{3}, m_{4}, l_{5}$ represent coordinate parameters and are not 0 .

The screw system consisting of $\$_{1}, \$_{2}, \$_{3}, \$_{4}$, and $\$_{5}$ has been simplified to:

$$
\left(\begin{array}{ccc:ccc}
1 & 0 & 0 & 0 & 0 & 0 \\
0 & 1 & 0 & 0 & 0 & 0 \\
0 & 0 & 0 & l_{5} & 0 & 0 \\
0 & 0 & 0 & 0 & m_{4} & 0 \\
0 & 0 & 0 & 0 & 0 & n_{3}
\end{array}\right)
$$

Finally, the reciprocal screw of the constrained limb

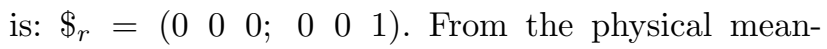
ing of the screw and the reciprocal screw, when the screw represents motion, the reciprocal screw represents constraints, that means, the constraint limb gives the moving platform a constraint moment rotating around the $z$-axis, while the remaining degrees of free-

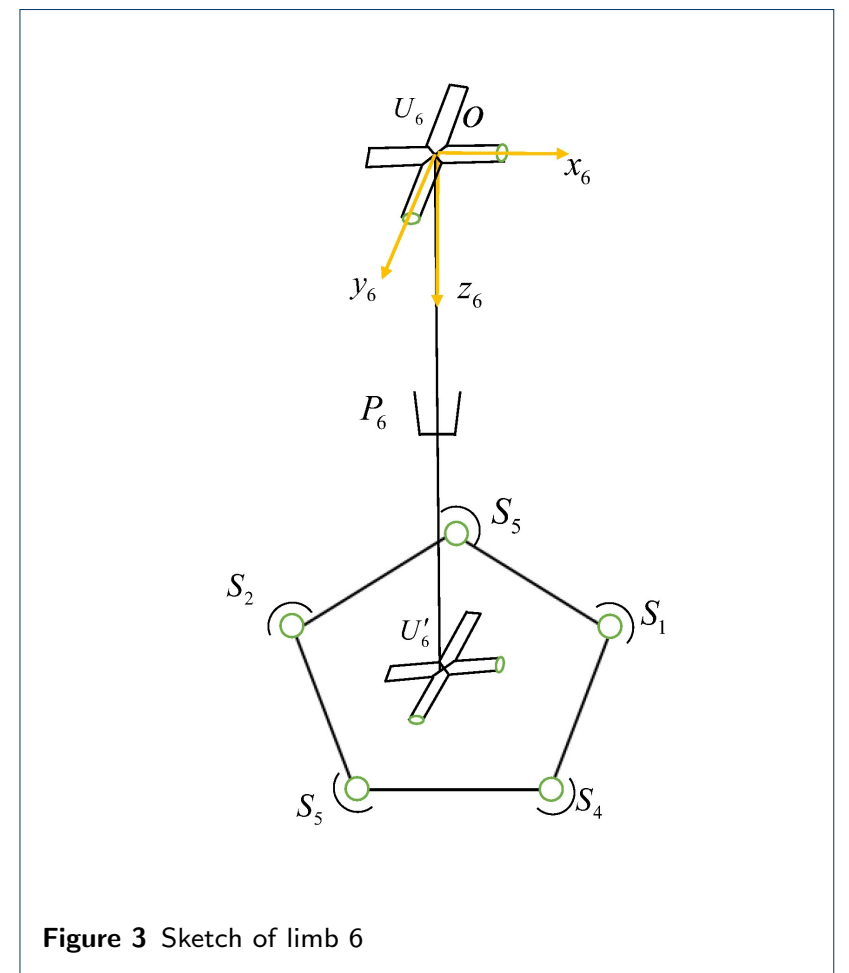

dom are unrestricted. That is, the moving platform can achieve the movement of five degrees of freedom - moving along the $x$-axis, $y$-axis, $z$-axis and rotating around the $x$-axis and $y$-axis.

\subsubsection{Experimental verification}

After the above scheme of 5-DOF parallel mechanism is verified by Grubler-Kutzbach and screw theory, the prototype is built for experiment in this study. The frame of the prototype is built with the 20millimetre-long profiles. The screw module and profiles are connected by connecting plates. The 5-DOF parallel mechanism is composed of a moving platform, upper and lower plates, ball screws, driving limbs, restrainting limb, connecting plates, Hook joints and spherical hinges, as shown in Figure 4. In this paper, the lower plate is the fixed platform, and the geometric center of the fixed platform is set as the zero position. However, after the construction of the prototype, when the moving platform is directly above the zero point, it is found that the movement of the moving platform is 
unstable and the degree of freedom is uncertain. The experimental results show that the scheme needs to be improved.

\subsubsection{Problem analysis of parallel mechanism}

Parallel mechanism has the characteristics of strong bearing capacity, large stiffness and high motion accuracy. However, in practical application, it appears stiffness degradation, motion instability, bearing capacity reduction and other phenomena when it is at some special positions, which is revealed in the experimental verification. In the study of this paper, the uncertainty of degree of freedom appears when the moving platform is directly above the zero point. The reduction of mechanism performance is not only related to the design, manufacture and assembly of the mechanism, but also closely related to the unique singularity and the dynamic stability of the singularity. The singularity of the mechanism generally refers to the situation that the inverse kinematics solution of the mechanism does not exist, the mechanism motion is unstable, and the driving force in the limb suddenly increases at some positions [30]. The analysis of singularity is the basis to verify the design and avoid the motion in singular space.

\subsection{Singularity analysis}

The essence of singularity of parallel mechanism is force screw failure of partial limbs acting on the moving platform. Based on this, the force of limbs acting on the moving platform is defined as line vector. The linear correlation between these line vectors is analyzed by using Grassmann line geometry method and the maximum linear independent number of these line vectors is defined as rank of line cluster.

The rank of the line cluster can be divided into 1 to 5 [31]. When the rank of the line cluster is 1 , there is a line in the three-dimensional space. When the rank of a line cluster is 2 , it includes two types of spatial dis- tribution: (a) A pair of skew lines; (b) A flat pencil of lines. When the rank of the line cluster is 3 , there are 4 kinds of spatial distribution: (a) A regulus; (b) All lines through a point; (c) The union of two flat pencils having a line in common but lying in distinct planes and with distinct centers; (d) All lines in a plane. When the rank of the line cluster is 4 , the spatial distribution is 4 kinds: (a) Elliptic congruence; (b) Parabolic congruence; (c) Hyperbolic congruence; (d) Degenerate congruence. When the rank of the line cluster is 5, there are two spatial distributions: (a) Non singular complex: generated by five skew lines ; (b) Singular complex: all the lines meeting one given line.

The singularity of this scheme occurs when the moving platform is parallel to the fixed platform and the moving platform is directly above the zero point, the force line vector extension lines of the five limbs intersect at one point, as shown in Figure 5. In this case, the singular form of the rank of the line cluster belongs to the (b) spatial distribution with rank 3 of the line cluster. It is the case that all lines through a point. At this time, the rank of the five force line vectors is 3 . In theory, for a compound screw system composed of force line vectors and couple-vectors, if the force line vectors are linearly independent of each other, and the couple-vectors are independent of each other, after the force line vectors are translated, the new force couple system composed of the resulting additional force couple and the original force couple system is also linearly independent. However, the specific research object of this paper is five force line vectors and a couple-vector. From the specific form of plücker coordinate, the rank of the composite screw system is equal to the rank of a directional vector matrix composed of five force line vectors plus one couple-vector rank [32]. Therefore, the rank of the five force line vectors is 3 , plus the rank 1 of the couple-vector, so that the rank of the final composite screw system is 4 . The corresponding physical 


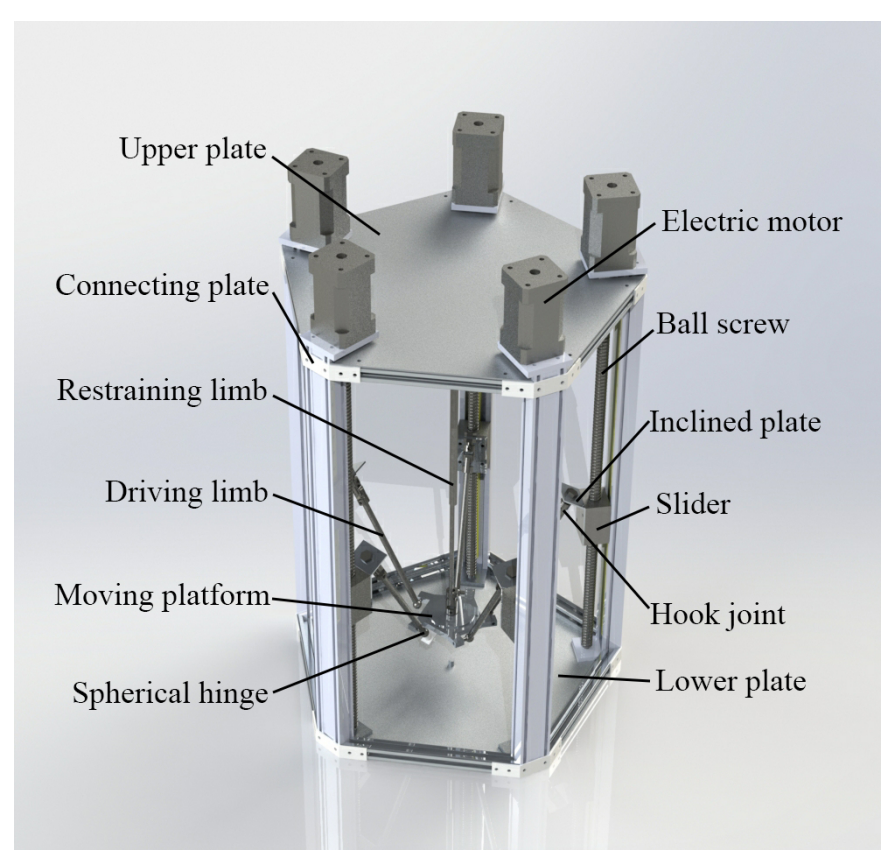

(a) three-dimensional model

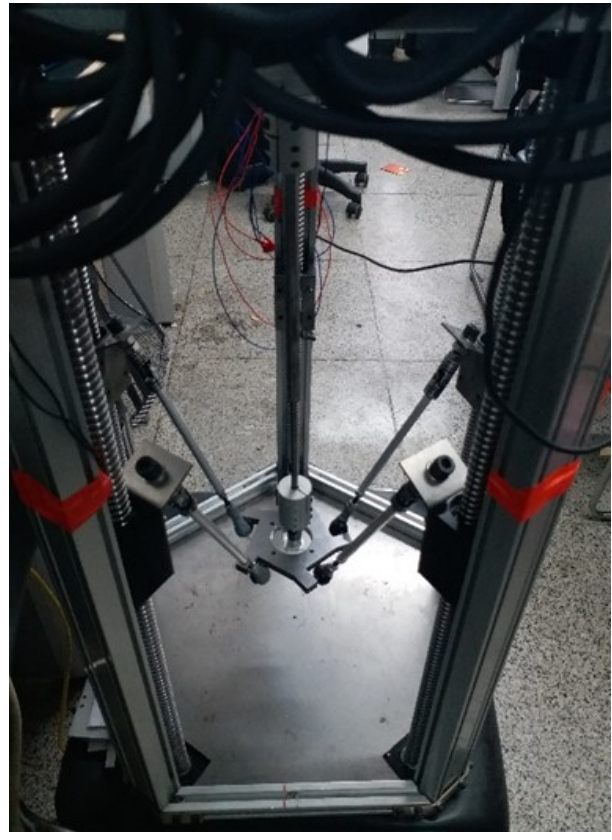

(b) physical prototype

Figure 4 three-dimensional model and physical prototype

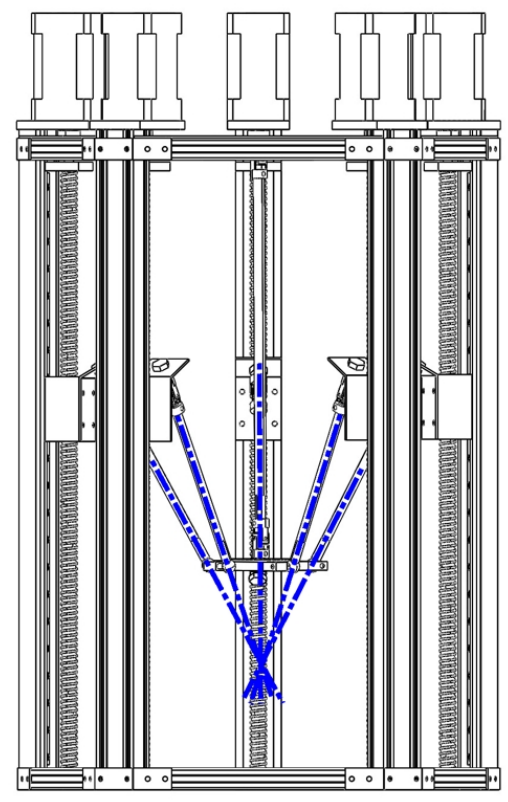

Figure 5 Singular position of preliminary scheme

meaning is that the movement of the moving platform along the $X, Y$ and $Z$ axes and the rotation around the $Z$-axis are effectively constrained while the rotation around the $X$ and $Y$ axes is not limited, resulting in a singularity. This kind of singularity is called the "structural singularity" of the mechanism since both fixed and moving platforms are regular pentagons.

\subsection{Scheme improvement and singularity analysis}

\subsubsection{Scheme improvement}

Based on the singular defect caused by the same shape of fixed platform and moving platform in the preliminary scheme, the moving platform is changed from pentagon to quadrilateral. The mechanism sketch is shown in Figure 6.

In the improved scheme, the fixed platform also uses 5 mobile pairs as input and presents a positive pentagonal symmetrical distribution in space, while the moving platform uses a rectangular structure. 


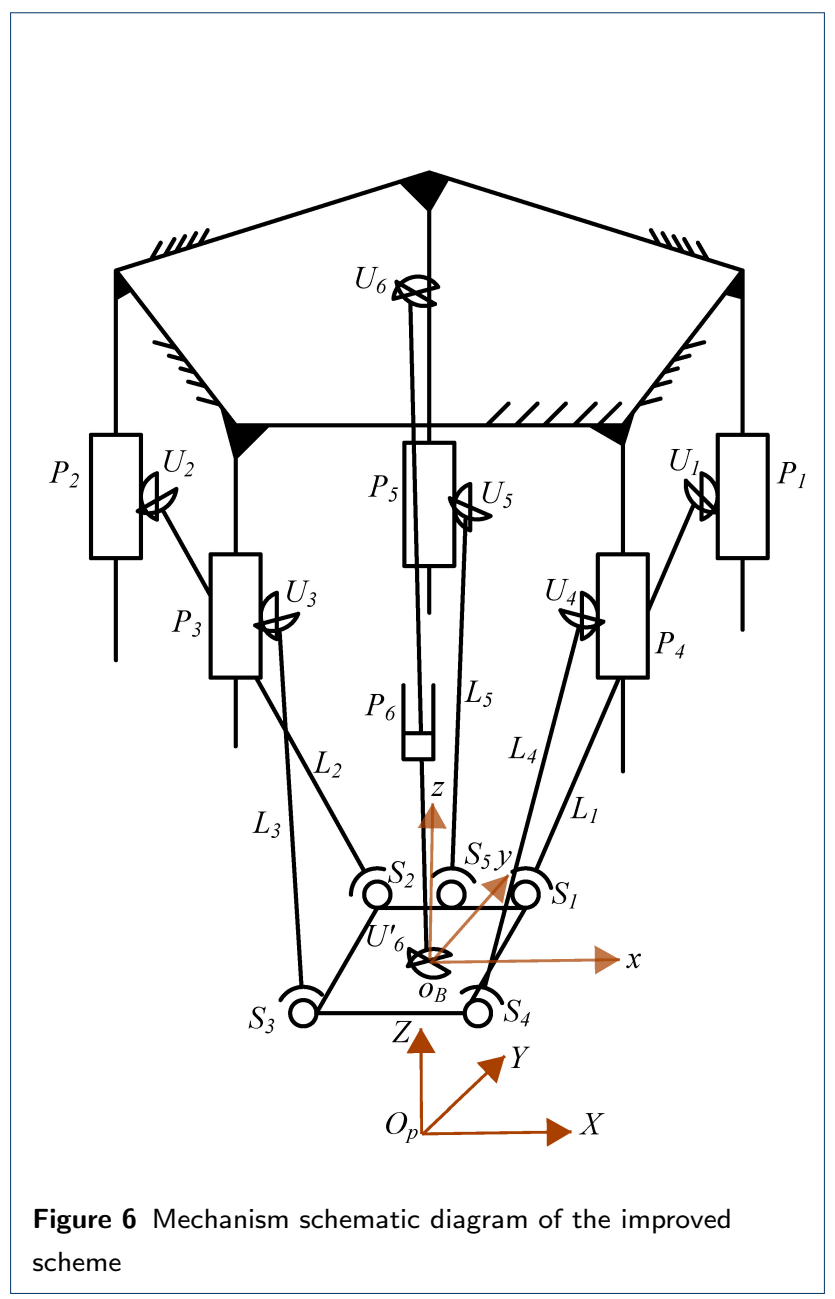

\section{Kinematic analysis}

\subsection{Establishment of inverse kinematics equations}

The coordinate systems of the mechanism consists of a moving coordinate system and a fixed coordinate system, as shown in Figure 6. The transformation matrix 


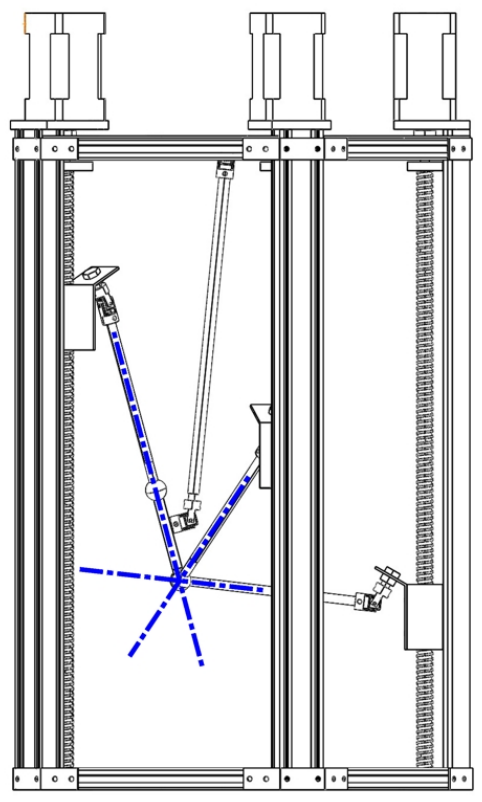

Figure 7 Singularity of improved scheme

of the moving coordinate system $o_{b}-x y z$ relative to the fixed coordinate system $O_{p}-X Y Z$ is: coordinate system can be converted into a specific coordinate value in the fixed coordinate system by

$$
\left(\begin{array}{l}
S_{i} \\
1
\end{array}\right)=T\left(\begin{array}{l}
s_{i} \\
1
\end{array}\right)
$$

Where $S_{i}$ is the coordinate value of 5 spherical hinges in the fixed coordinate system; $s_{i}$ is the coordinate value of 5 spherical hinges in the fixed coordinate system, and $T$ is the coordinate transformation matrix.

The coordinate values of Hook joint $U_{i}(i=1 \sim 5)$ on the fixed platform and spherical hinge $S_{i}(i=1 \sim 5)$ on the moving platform in their respective coordinate systems are obtained through geometric relationship. Then the coordinate values of each point of the moving platform $S_{i}(i=1 \sim 5)$ in the fixed coordinate system can be obtained from Eqs. (8). During the movement of the moving platform, the link length remains unchanged. Any drive limb has the following closed-loop vector diagram shown in Figure 8:

$$
\begin{aligned}
& T=\operatorname{Trans}(\mathrm{x}, \mathrm{y}, \mathrm{z}) \cdot \operatorname{Rot}(\alpha, \beta, \gamma) \\
& =\left(\begin{array}{cccc}
1 & 0 & 0 & x \\
0 & 1 & 0 & y \\
0 & 0 & 1 & z \\
0 & 0 & 0 & 1
\end{array}\right) \cdot\left(\begin{array}{cccc}
\cos \beta & \sin \beta \sin \alpha & \sin \beta \cos \alpha & 0 \\
0 & \cos \alpha & -\sin \alpha & 0 \\
-\sin \beta & \cos \beta \sin \alpha & \cos \beta \cos \alpha & 0 \\
0 & 0 & 0 & 1
\end{array}\right) \\
& =\left(\begin{array}{cccc}
\cos \beta & \sin \beta \sin \alpha & \sin \beta \cos \alpha & x \\
0 & \cos \alpha & -\sin \alpha & y \\
-\sin \beta & \cos \beta \sin \alpha & \cos \beta \cos \alpha & z \\
0 & 0 & 0 & 1
\end{array}\right)
\end{aligned}
$$

Where $x, y$ and $z$ represent the moving distance of the moving coordinate system along the $X, Y$ and $Z$ axes of the fixed coordinate system respectively; $\alpha, \beta$ and $\gamma$ represent the rotating angles of the coordinate system along the $X, Y$ and $Z$ axes of the fixed coordinate system respectively. Any point in the moving

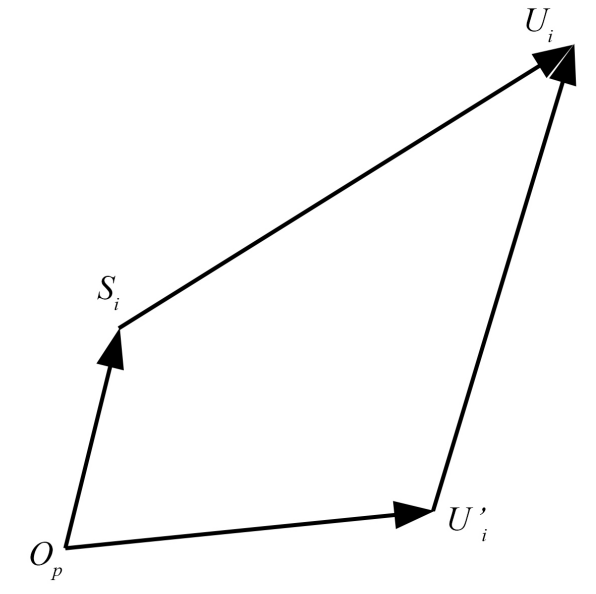

Figure 8 Closed-loop vector diagram 
Closed loop vector equation is established for Figure 8:

$$
\overline{S_{i} U_{i}}=\overline{O_{p} U_{i}^{\prime}}+\overline{U_{i}^{\prime} U_{i}}-\overline{O_{p} S_{i}}, i=1,2, \ldots 5
$$

Among them, $O_{p}$ is the origin of fixed coordinate system, $S_{i}$ is the geometric center of spherical hinge on moving platform, $U_{i}$ is the geometric center of Hook joint fixed on slider, $U_{i}^{\prime}$ is the projection of $U_{i}$ on the plane of fixed coordinate system $X O Y$, and the modulus of the vector corresponds to the length from the center of the Hook joint to the center of the spherical hinge on each driving limb of $L_{1} \sim L_{5}$, and this length is a fixed value during the movement of the moving platform.

$L_{i}=\left|\overline{S_{i} U_{i}}\right|=\sqrt{\left(X_{i}^{U}-X_{i}^{S}\right)^{2}+\left(Y_{i}^{U}-Y_{i}^{S}\right)^{2}+\left(Z_{i}^{U}-Z_{i}^{S}\right)^{2}}$

Where $X_{i}^{U}, Y_{i}^{U}$, and $Z_{i}^{U}$ represent the $X, Y$ and $Z$ coordinates of $U_{i} ; X_{i}^{S}, Y_{i}^{S}$, and $Z_{i}^{S}$ represent the $X$, $Y$ and $Z$ coordinates of $S_{i}$.

The mapping equation for the position of five driving sliders and the posture coordinates of the moving platform is simplified as follows:

$$
Z_{i}^{U}=Z_{i}^{S} \pm \sqrt{L_{i}^{2}-\left(X_{i}^{U}-X_{i}^{S}\right)^{2}+\left(Y_{i}^{U}-Y_{i}^{S}\right)^{2}}
$$

Specific inverse equations can be obtained by incorporating coordinate values of $U_{i}$ and $S_{i}$. Considering that the five sliders must be above the moving platform during the movement, the position inverse solution equation only takes positive signs.

The velocity equation of the mechanism is obtained by taking the derivative of Eq. (12) with respect to time. Similarly, the acceleration equation of the mech- anism is obtained by taking the derivative of the velocity equation with respect to time.

$$
\left(\begin{array}{l}
\dot{U}_{1 z} \\
\dot{U}_{2 z} \\
\dot{U}_{3 z} \\
\dot{U}_{4 z} \\
\dot{U}_{5 z}
\end{array}\right)=\left(\begin{array}{ccccc}
\frac{\partial U_{1 z}}{\partial x} & \frac{\partial U_{1 z}}{\partial y} & \frac{\partial U_{1 z}}{\partial z} & \frac{\partial U_{1 z}}{\partial \alpha} & \frac{\partial U_{1 z}}{\partial \beta} \\
\frac{\partial U_{2 z}}{\partial x} & \frac{\partial U_{2 z}}{\partial y} & \frac{\partial U_{2 z}}{\partial z} & \frac{\partial U_{2 z}}{\partial \alpha} & \frac{\partial U_{2 z}}{\partial \beta} \\
\frac{\partial U_{3 z}}{\partial x} & \frac{\partial U_{3 z}}{\partial y} & \frac{\partial U_{3 z}}{\partial z} & \frac{\partial U_{3 z}}{\partial \alpha} & \frac{\partial U_{3 z}}{\partial \beta} \\
\frac{\partial U_{4 z}}{\partial x} & \frac{\partial U_{4 z}}{\partial y} & \frac{\partial U_{4 z}}{\partial z} & \frac{\partial U_{4 z}}{\partial \alpha} & \frac{\partial U_{4 z}}{\partial \beta} \\
\frac{\partial U_{5 z}}{\partial x} & \frac{\partial U_{5 z}}{\partial y} & \frac{\partial U_{5 z}}{\partial z} & \frac{\partial U_{5 z}}{\partial \alpha} & \frac{\partial U_{5 z}}{\partial \beta}
\end{array}\right) \cdot\left(\begin{array}{c}
\dot{x} \\
\dot{y} \\
\dot{z} \\
\dot{\alpha} \\
\dot{\beta}
\end{array}\right)
$$

Where $U_{i z}(i=1,2, \ldots, 5)$ represents the coordinate of the $Z$ axis of the five sliders in the static coordinate system.

\subsection{Algorithm simulation}

In this mechanism, radius of outer circle of geometric center of Hook joint fixed on five sliders $R=135 \mathrm{~mm}$. The distance from the spherical hinge to the geometric center of Hook joint $L_{i}=205 \mathrm{~mm}$. The coordinate values of the five spherical hinges rotation centers in the moving coordinate system are $x_{1}=35 \mathrm{~mm}, y_{1}=$ $51 \mathrm{~mm}, x_{2}=-35 \mathrm{~mm}, y_{2}=51 \mathrm{~mm}, x_{3}=-51 \mathrm{~mm}$, $y_{3}=-35 \mathrm{~mm}, x_{4}=51 \mathrm{~mm}, y_{4}=-35 \mathrm{~mm}, x_{5}=0$ $\mathrm{mm}$ and $y_{5}=51 \mathrm{~mm}$. On the premise of $x=0$ and $\beta=0^{\circ}$, the trajectory curve equation of the center point of the moving platform is:

$$
\left\{\begin{array}{l}
\alpha=\frac{\pi}{3}+\frac{1}{30} \pi t,(0<t<10) \\
y=50 \cos \alpha \\
z=-50 \sin \alpha
\end{array}\right.
$$

The geometric center target track of the moving platform is shown Figure 9.

The position change trajectories of the five sliders corresponding to this condition are obtained by inverse position solution formula as shown in Figure 10. The simulation results of the position curve show that the position change curves of the five sliders on the ball screw can be obtained by inverse position solution 


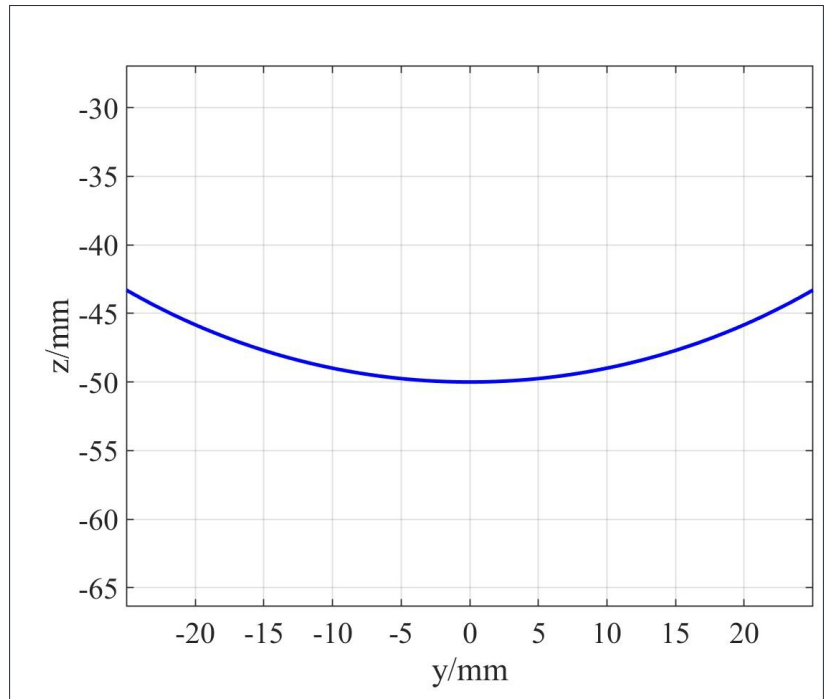

Figure 9 The motion path of the moving platform

equation as long as the motion path of the moving platform is known, which lays a foundation for the control of the mechanism.

The velocity curves of the five sliders under this condition are obtained by taking the derivative of the inverse position equations with respect to time, as shown in Figure 11.

The acceleration curves of the five sliders under this condition are obtained by taking the second derivative of the inverse position equations, as shown in Figure 12.

The acceleration simulation results of five sliders show that the position, speed and acceleration images of the sliders correspond to each other, and the absolute value of acceleration increases when the slope of the speed curve increases. The maximum absolute acceleration of the five sliders is $0.47 \mathrm{~mm} / \mathrm{s}^{2}$, and this position is the end of the curve. The larger acceleration value is caused by the smaller angle between the fifth limb and the $X O Y$ plane of the fixed coordinate system. If the movement continues according to this trend, the fifth limb will be parallel to the fixed platform, and a singularity will occur. Therefore, when planning the target curve of the moving platform, the

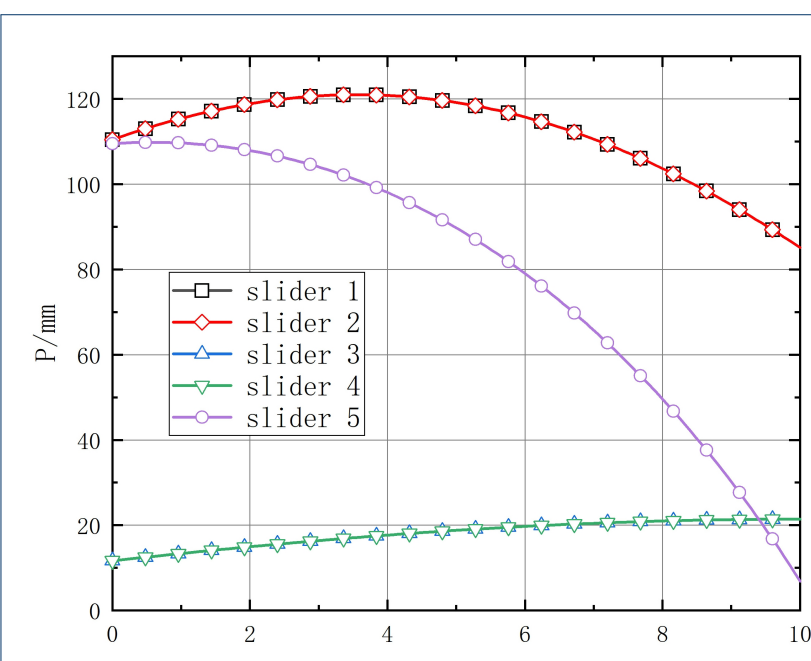

Figure 10 Position curves of sliders

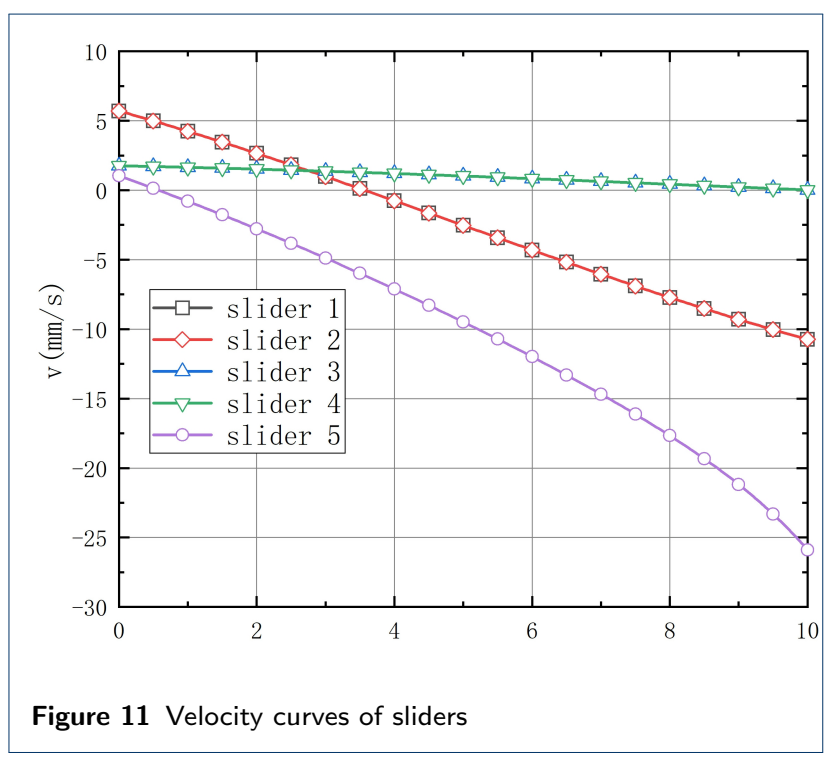




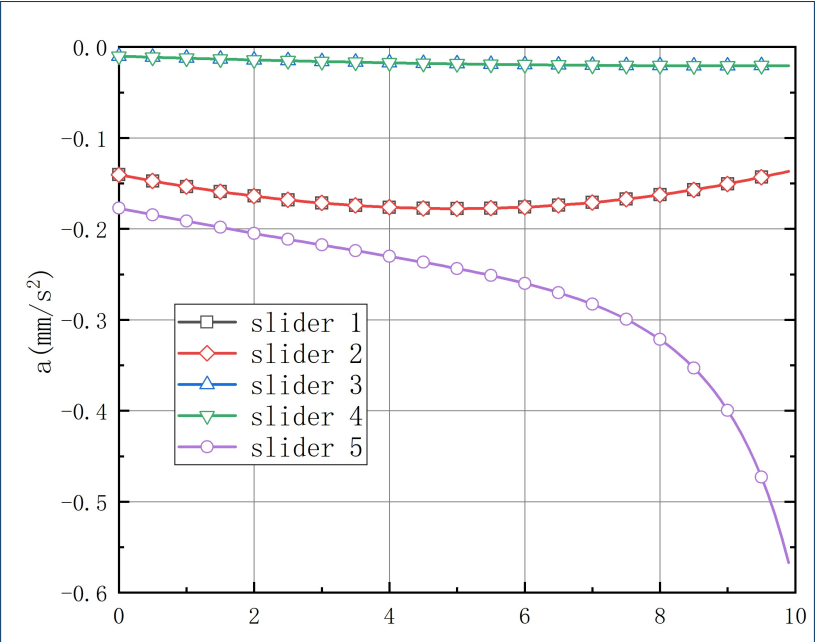

Figure 12 Acceleration curves of sliders driving motor move to a fixed limit position in the coordinate system of the parallel mechanism. The fixed limit position is a reference point of the zero point of the parallel mechanism, and is a fixed value relative to the origin of the fixed platform mechanism. The specific method is to set the motor speed so that the five sliders move in the same direction and at the same speed. If the sliders move to the limit position first, they will stop moving. Until all the sliders move to the corresponding limit position, the mechanism will return to the zero reference point.

The position of the reference point is determined by the position of the zero switch installed on the lead screw module. When the zero return button on the control panel is triggered, each driving shaft starts to seek zero along the positive direction of $Z$ axis and at the same speed, and the moving platform moves in the positive direction of $Z$ axis. When a drive shaft triggers the zero switch, a command is sent to control the shaft to stop moving. Until the five drive shafts reach the reference point, the moving platform is in a fixed position, and this position is directly above the true zero point of the parallel mechanism. Then, drive 5 motors to move down a fixed distance to make the moving platform coincide with the zero point of the parallel mechanism. At this time, clear the position information of each drive shaft through the zero clearing function to complete the return home operation.

\section{Workspace analysis}

\subsection{Influencing factors of workspace}

The main factors that affect the workspace of parallel robot are:

(1) The limitation of the length of link. The link lengths of each limb is not random and must be limited to a certain range. When the length of the link is the minimum, the moving platform and the five limbs are in the same plane, and the mechanism has a singularity. When the length of the link is the maximum, in the control program, the five sliders driven by the 


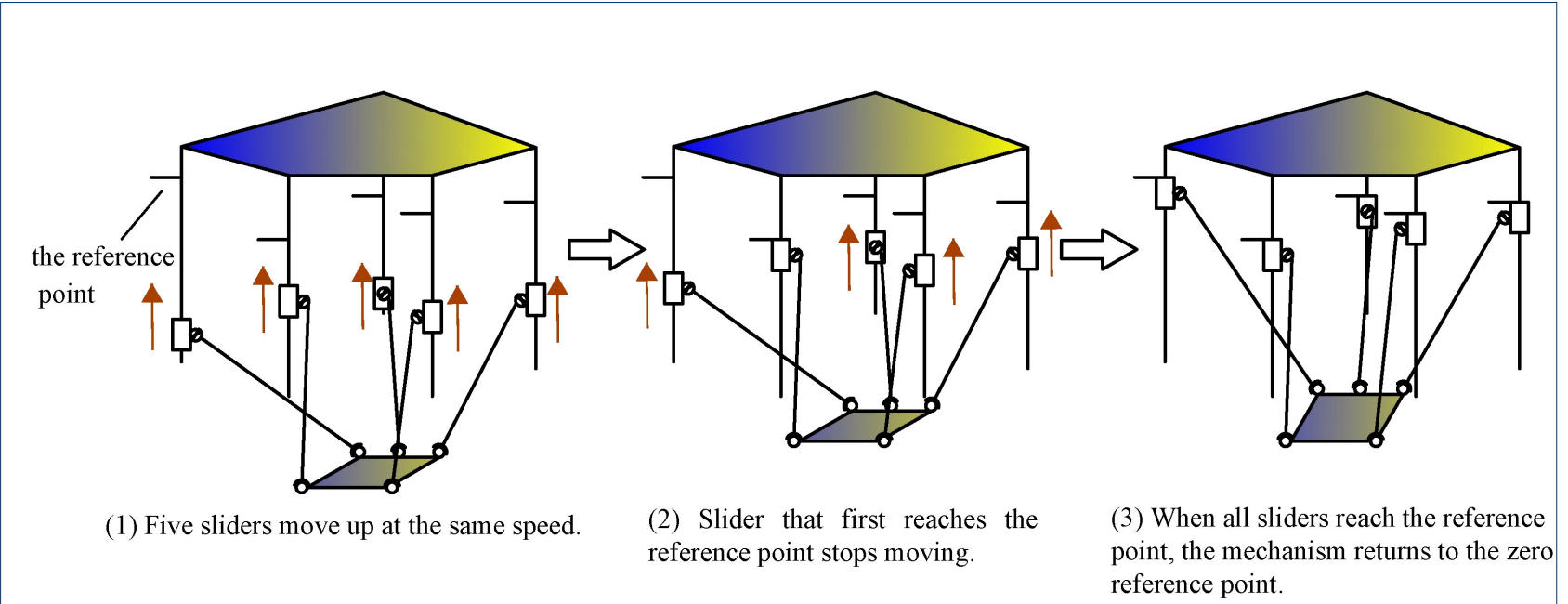

Figure 13 Diagram of go home process

the moving platform and the fixed platform coincide, the five sliders are located at the top of the lead screw, and the whole mechanism is locked.

(2) Limit of slider stroke. The influence of slider stroke range on workspace is mainly concentrated on $Z$ dimension of workspace.

(3) The limitation of the position and angle of the spherical hinge. Because the movement range of the spherical hinge is in a certain range, and the change of the position of the spherical hinge arrangement will change the position where the spherical hinge reaches the limit.

(4) Limit of link interference. In the actual prototype, each link has a certain size, so interference should be avoided.

\subsection{Monte Carlo method}

Monte Carlo method is based on the idea of random sampling to solve mathematical problems. The flowchart of solving mechanism workspace by Monte Carlo method is shown in Figure 14. The analysis process is as follows:

Step 1. The random function is used to traverse the value in the initially set workspace.
Step 2. These values are introduced into the inverse kinematics solution Eq. (12) to obtain the variable values of each kinematic pair.

Step 3. Record the corresponding traverse points of each motion pair in the workspace. These recorded traverse points are the points that the geometric center of the moving platform can reach, and finally constitute the threedimensional point cloud map of the workspace.

\subsection{Algorithm simulation of optimal workspace}

(1) Based on the influencing factors of workspace, the simulation parameters are changed to obtain the optimal workspace. The parameters are set as follows: the geometric centers of Hook joints on sliders are evenly distributed on the circle with radius $R=135 \mathrm{~mm}$. Since the length of link affects the size of workspace, the length of five driving links is set as $L=165 \mathrm{~mm}$, $185 \mathrm{~mm}, 205 \mathrm{~mm}$ and $225 \mathrm{~mm}$ respectively, and the projection of workspace on the $X O Y$ plane is shown in Figure 15.

From the comparison of Figure 15(a)-(d), it can be seen that when the length of the link is changed from $165 \mathrm{~mm}$ to $205 \mathrm{~mm}$, the projection of the threedimensional workspace on the $X O Y$ plane is signifi- 


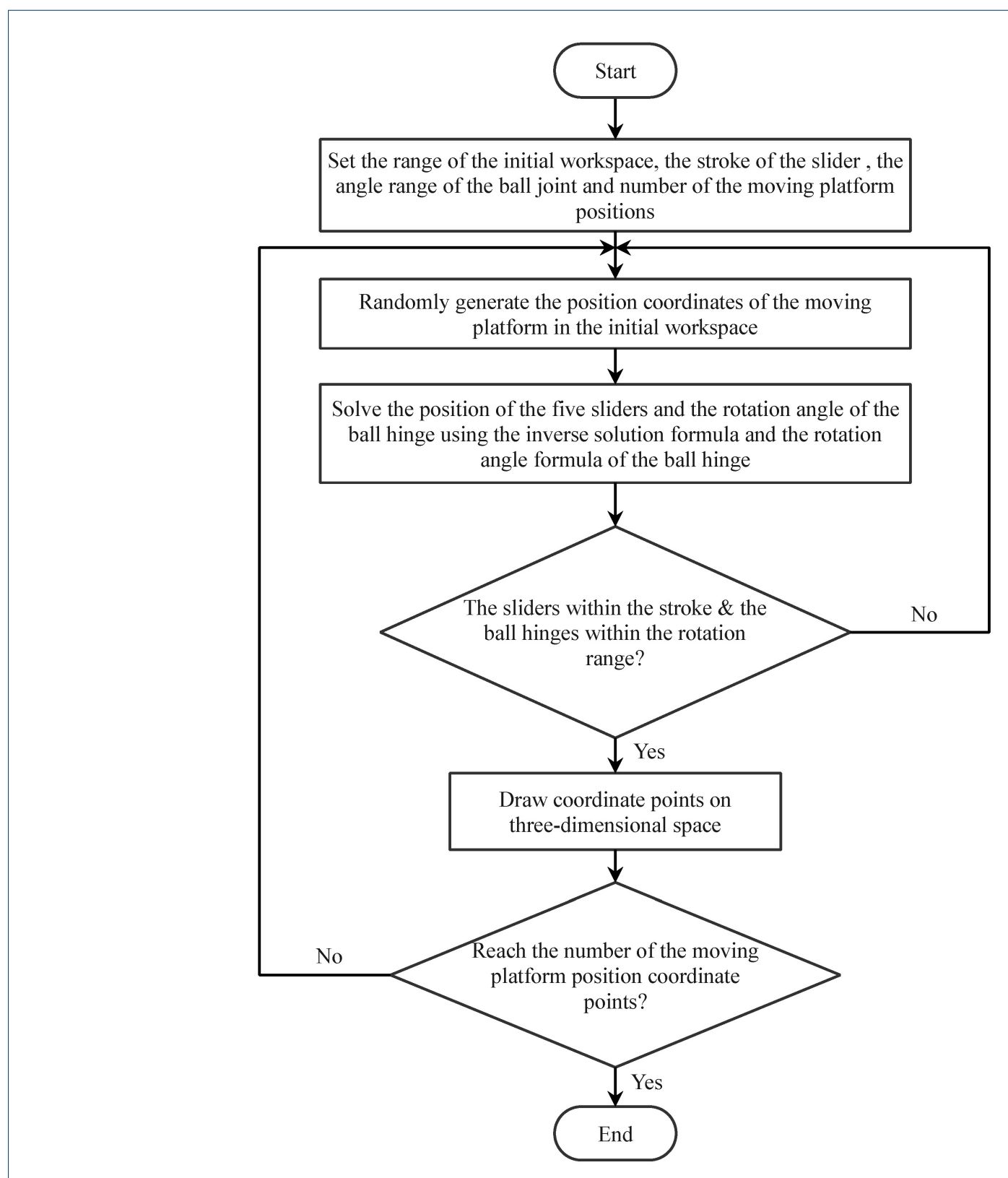

Figure 14 The flowchart of solving mechanism workspace by Monte Carlo method 


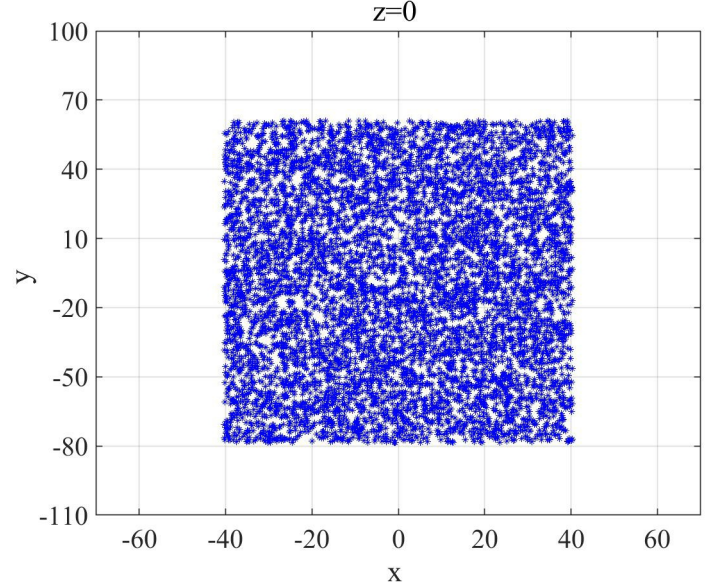

(a) $L=165 \mathrm{~mm}, Z=0 \mathrm{~mm}$

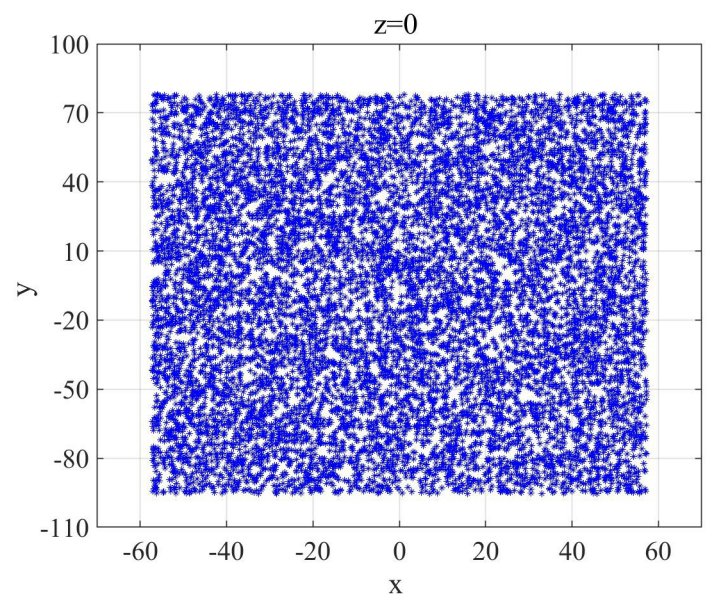

(c) $L=205 \mathrm{~mm}, Z=0 \mathrm{~mm}$

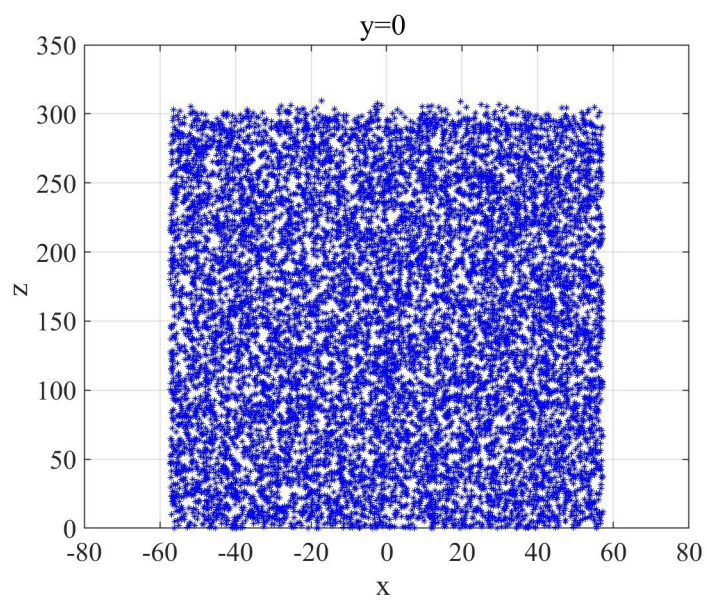

(e) $L=205 \mathrm{~mm}, Y=0 \mathrm{~mm}$

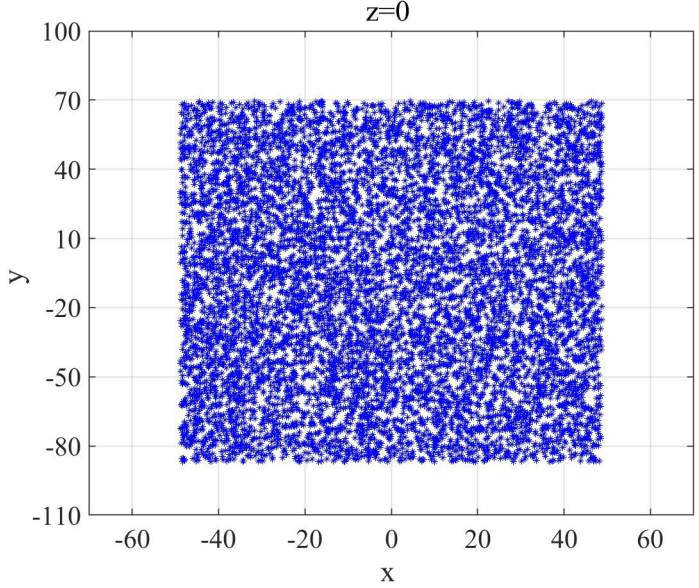

(b) $L=185 \mathrm{~mm}, Z=0 \mathrm{~mm}$

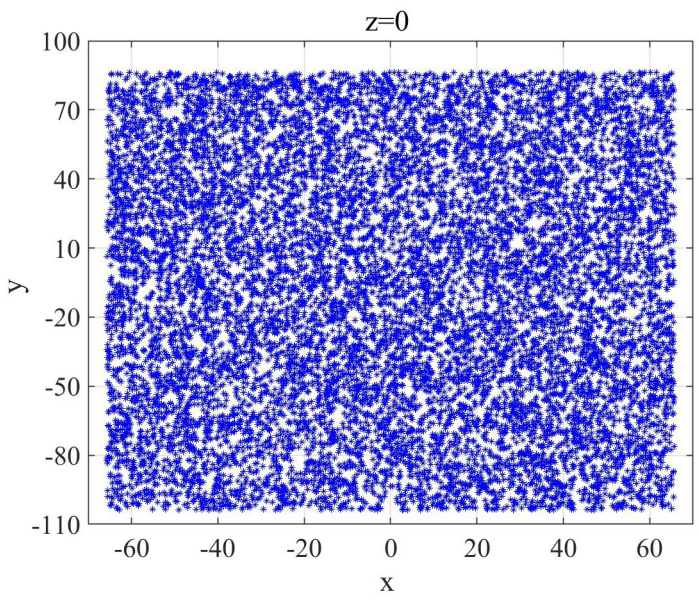

(d) $L=225 \mathrm{~mm}, Z=0 \mathrm{~mm}$

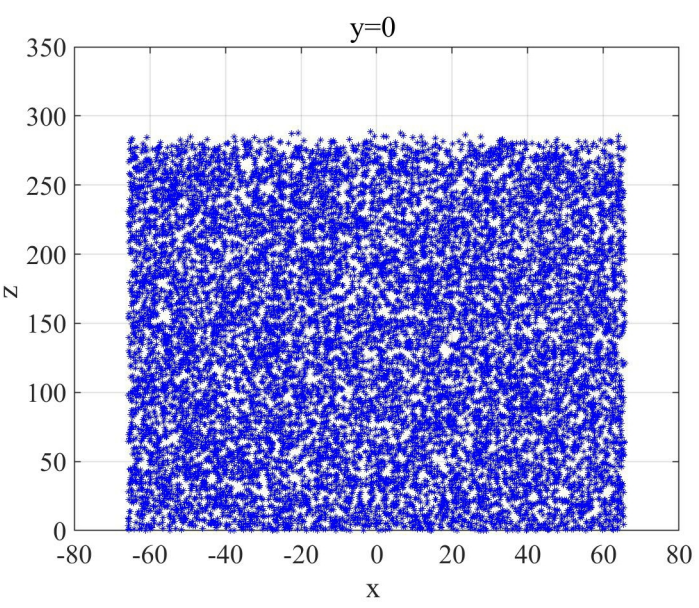

(f) $L=225 \mathrm{~mm}, Y=0 \mathrm{~mm}$

Figure 15 Limitation of link length interference 
cantly larger, but there is no obvious change from 205 $\mathrm{mm}$ to $225 \mathrm{~mm}$. Figure 15(e) and Figure 15(f) show the projection of the three-dimensional workspace on the $X O Z$ plane when the $\alpha, \beta$, and $Y$ coordinates of the moving platform are all zero. After comparison, it is found that when $L=205 \mathrm{~mm}$, the workspace has a better $Z$ direction space. Therefore, we choose the link length of the limb to be $205 \mathrm{~mm}$.

(2) After determining the length of the link $L=205$ $\mathrm{mm}$, the position of spherical hinge is optimized as shown in Figure 16 and the simulation result is shown in Figure 17.

When the length of the link and distribution radius of the Hook hinge are the same, and the spherical hinge is distributed according to Figure 16(a), the projected area of the working space on the $X O Y$ plane is $120 \mathrm{~mm} \times 180 \mathrm{~mm}$. When the spherical hinge is arranged according to Figure 16(b), the projection area of the corresponding workspace in the $X O Y$ plane is $160 \mathrm{~mm} \times 120 \mathrm{~mm}$, and the workspace is heavily biased to the negative direction of the $Y$ axis, as shown in Figure 17(b). Comparing the two schemes of spherical hinge, it is found that scheme 1(Figure 16(a)) has better isotropy in the workspace of $X O Y$ plane, so this scheme will be adopted in the subsequent mechanism construction, and the previous chapters are based on the analysis of this solution.

\section{(3) Fixed posture workspace}

As an important form of workspace, fixed posture workspace studies the size of workspace when the moving platform is in a fixed posture. When $\alpha$ equals $5^{\circ}$, $10^{\circ}, 15^{\circ}$ and $20^{\circ}$, the fixed posture workspace is analyzed to study the influence of single posture angle on workspace. The results are shown in Figure 18.

Similarly, when $\beta$ changes from $5^{\circ}$ to $20^{\circ}$, the change in the fixed posture workspace is shown in Figure 19.
Finally, when both two posture angles are $0^{\circ}$, the size of the three-dimensional workspace is studied on the basis of the optimized length of the link and the position of the spherical hinge. The results are shown in Figure 20.

From Figure 18 and Figure 19, we can see that the 5-DOF parallel mechanism consists of two degrees of freedom of rotation, and the size of the two posture angles affects the size of the workspace, showing a general rule that the size of fixed posture workspace decreases with the increase of the posture angle and deviates from one direction of the coordinate axis. As to whether any variable attitude curve in space is within the workspace, we can reverse the kinematics of the subdivision points of the target curve to judge if the position of the sliders and the angle of spherical hinges corresponding to each subdivision point are within the range of motion.

The overlapping part of the simulation results of the fixed posture workspace satisfies the two cases, so according to the symmetry and superposition of the simulation results of $\alpha$ and $\beta$, the workspace size of the mechanism is $80 \mathrm{~mm} \times 150 \mathrm{~mm} \times 300 \mathrm{~mm}$, when $\alpha$ and $\beta$ are in the range of $\pm 5^{\circ}$. It is known from Figure 20 that when $\alpha=0^{\circ}$ and $\beta=0^{\circ}$, the size of the fixed posture workspace is $120 \mathrm{~mm} \times 175 \mathrm{~mm} \times 300 \mathrm{~mm}$.

\section{Experiment}

The whole prototype is made of profiles with side length of $20 \mathrm{~mm}$, and the lead screw module and profiles are connected by connecting plates. The whole 5 -DOF parallel mechanism is composed of moving platform, upper and lower plates, ball screws, driving limbs, restraining limb, connecting plates, Hook joints and spherical hinges. The lower plate in this paper is also called fixed platform. The 3D model and physical model are shown in Figure 21.

The size of the profile is shown in Figure 22; the diameter of the link is $8 \mathrm{~mm}$, the length of the link 


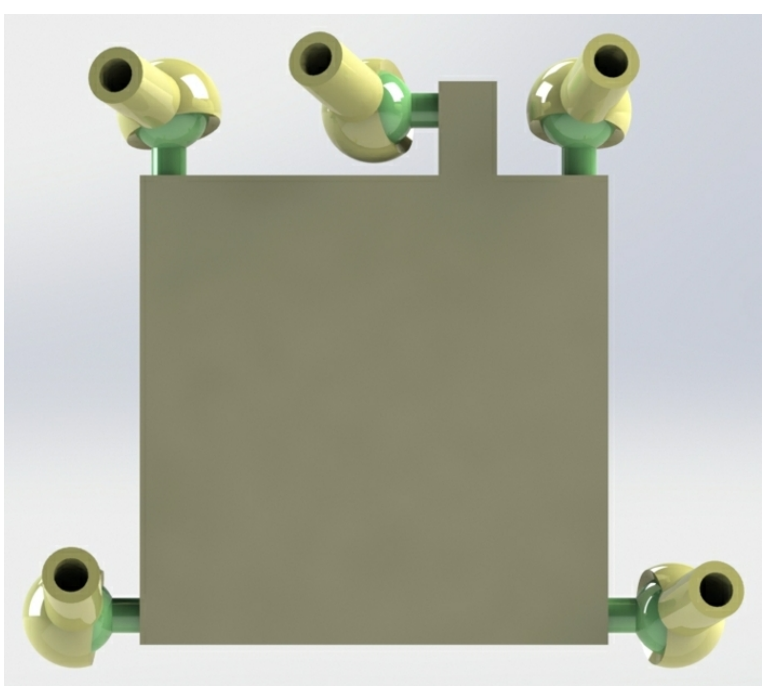

(a) Scheme 1

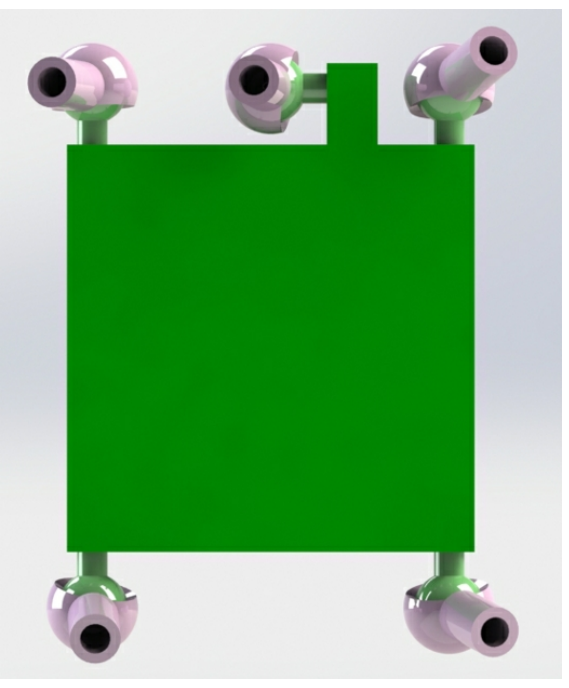

(b) Scheme 2

Figure 16 Distribution of spherical hinges on the moving platform

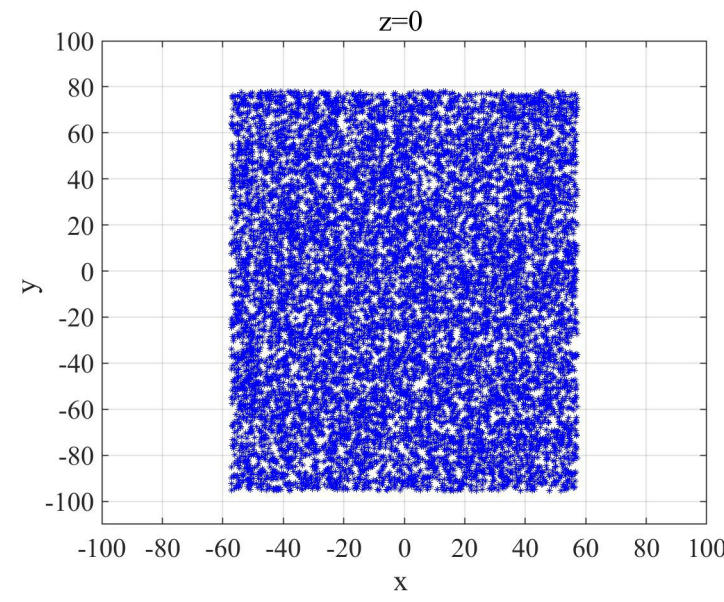

(a) Scheme 1

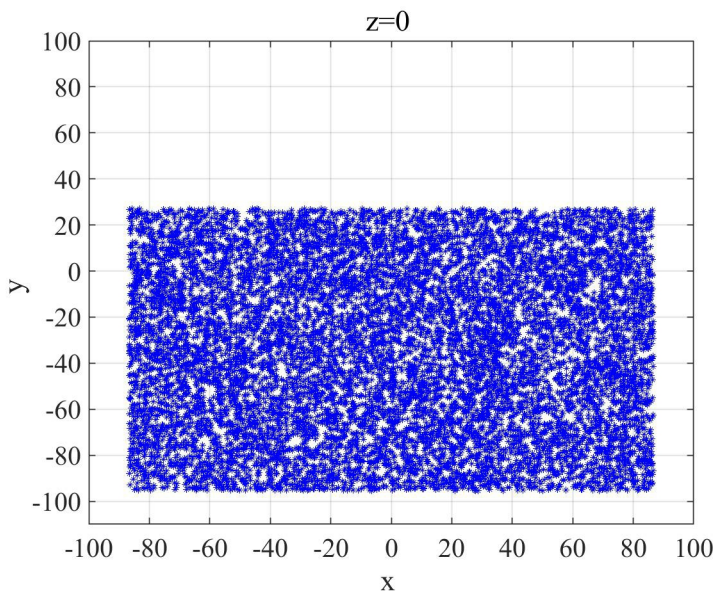

(b) Scheme 2

Figure 17 Workspace diagrams of the distribution of spherical hinges for schemes 1 and 2 


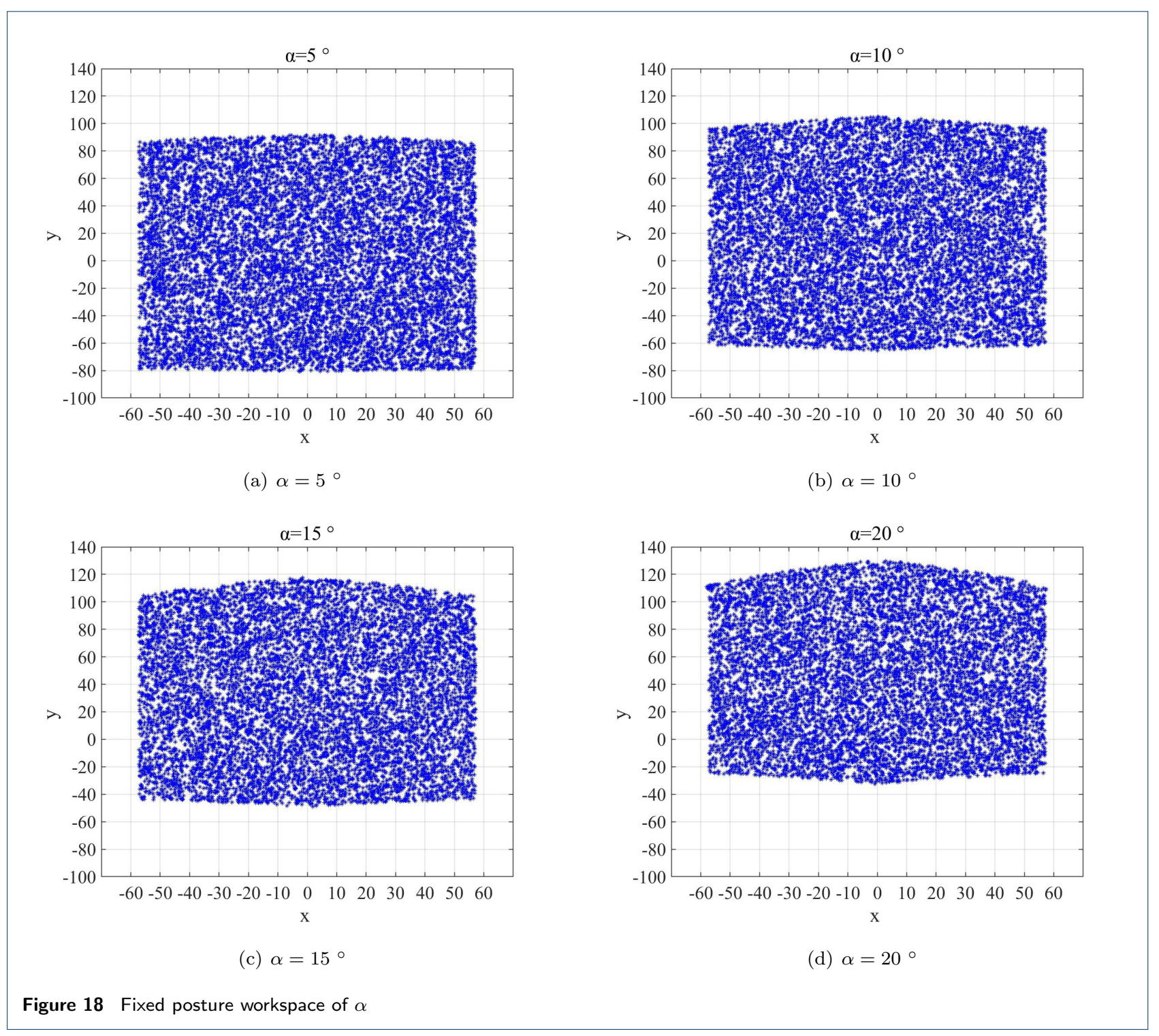




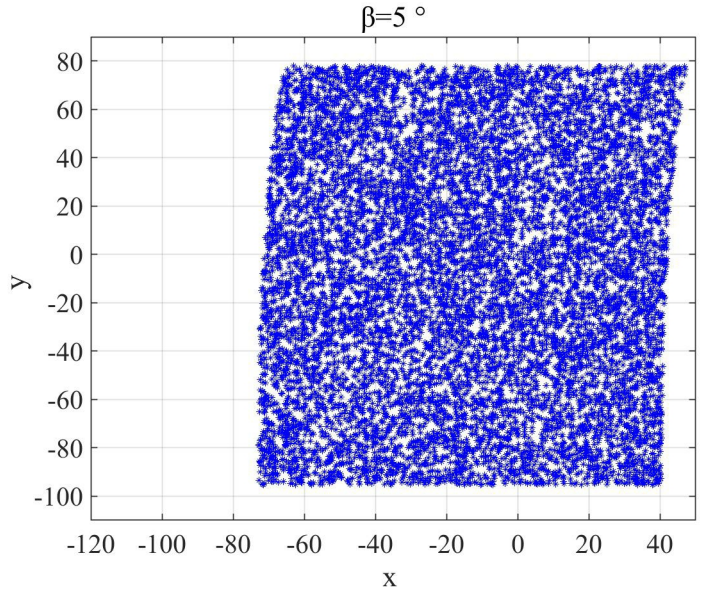

(a) $\beta=5^{\circ}$

$\beta=15^{\circ}$

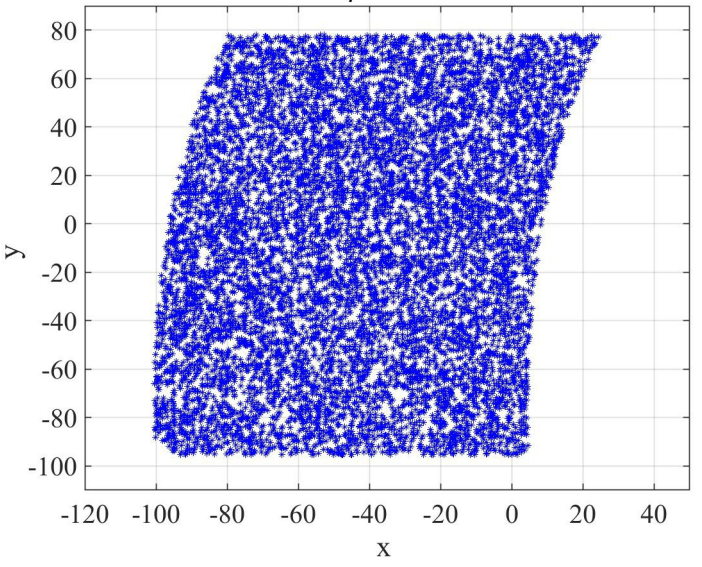

(c) $\beta=15^{\circ}$

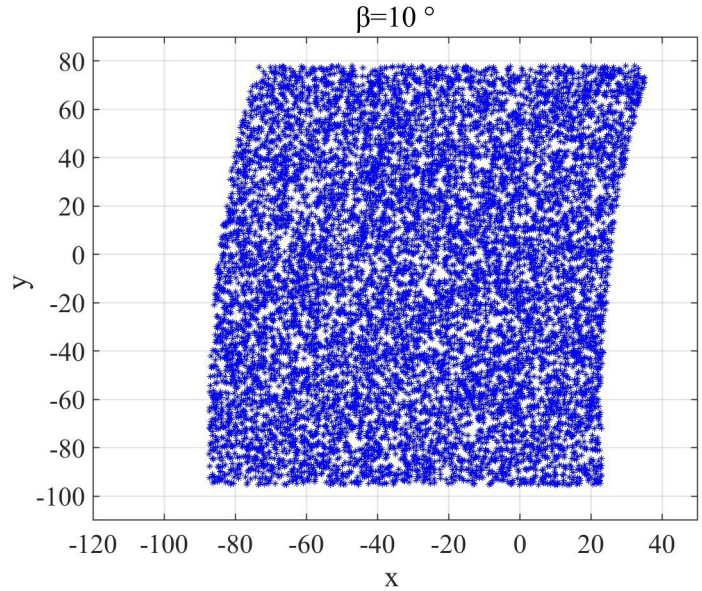

(b) $\beta=10^{\circ}$

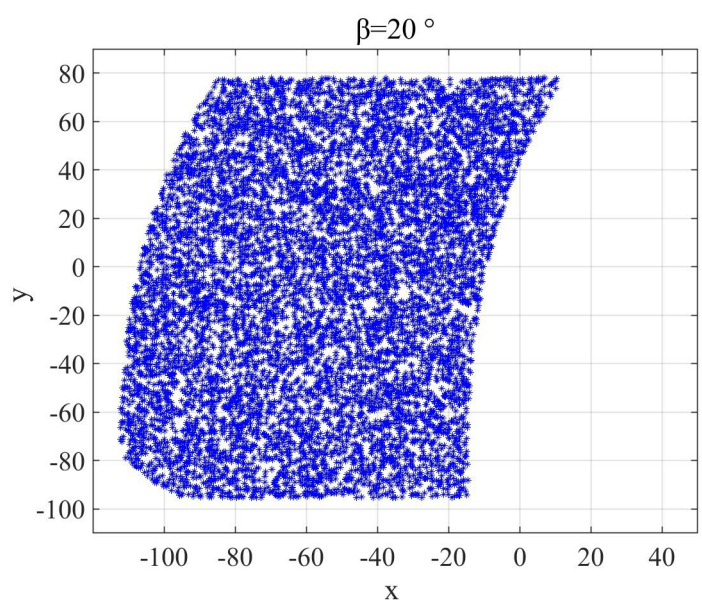

(d) $\beta=20^{\circ}$

Figure 19 Fixed posture workspace of $\beta$ 


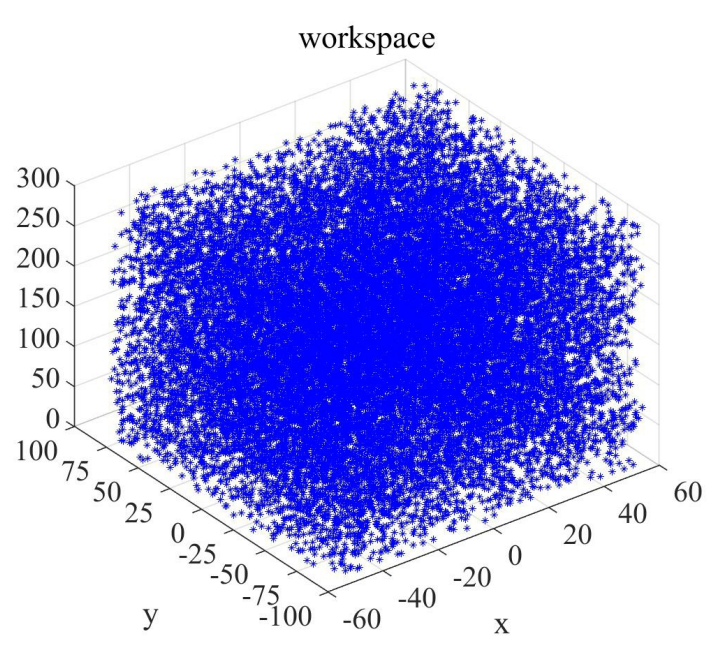

Figure 20 Fixed posture workspace

is $205 \mathrm{~mm}$; Diameter, lead and effective stroke of ball screw are $16 \mathrm{~mm}, 5 \mathrm{~mm}$ and $205 \mathrm{~mm}$ respectively; the base size of the moving platform is a square with a side length equal to $80 \mathrm{~mm}$, and the coordinates of the center positions of the five spherical hinges are $(35,51)$, $(-35,51),(-51,-35),(51,-35),(0,51)$; the slider is connected to the Hook joint on the driving limb through the inclined plate, and the angle between the two planes of the inclined plate is $120^{\circ}$. In addition, the center points of five Hook joints connected with the slider are evenly distributed on a circle with a radius of $135 \mathrm{~mm}$.

In order to check the degree of freedom of the mechanism and measure the motion accuracy of the moving platform actuator, the posture angles of the moving platform are now set to $\alpha=0^{\circ}, \beta=0^{\circ}$ and $\alpha=0^{\circ}, \beta=-5^{\circ}$. We use a pencil to replace the printing nozzle, and the axis of the pencil is required to be perpendicular to the plane of the drawing paper and a circle with a radius of $30 \mathrm{~mm}$ is drawn to verify the space motion function of the device. The track of the circle is planned and the reference speed of the moving platform is $10 \mathrm{~mm} / \mathrm{s}$.
During the experiment, the tip of the pen starts from the coordinate origin $o$. Firstly, the moving platform moves $5 \mathrm{~mm}$ in the positive direction of $Z$-axis, then moves to the right first above point $A$. The moving platform moves $5 \mathrm{~mm}$ in the negative direction of $Z$ axis and the pen tip falls at point $A$. Then, go through $B, C, D$ counterclockwise and back to point $A$. Finally, the moving platform moves up, the pen tip leaves the paper, and the experiment is completed. Among them, when drawing a circle with the posture angle $\alpha=0^{\circ}$ and $\beta=-5^{\circ}$ and the moving platform moves from directly above the origin to directly above point $A$, not only does the distance change, but also $\beta$ changes from $0{ }^{\circ}$ to $-5^{\circ}$ during this process. From this, the rotational freedom of the parallel mechanism can be verified.

The experimental results of drawing a circle in two situations with posture angles $\alpha=0^{\circ}, \beta=0^{\circ}$ and $\alpha=0^{\circ}, \beta=-5^{\circ}$ are shown in Figure 23 .

The coincidence point A of the circular trajectory can indicate that the trajectory of the parallel mechanism has good coincidence, and the successful drawing of circles in two cases of $\alpha=0^{\circ}, \beta=0^{\circ}$ and $\alpha=0{ }^{\circ}, \beta=-5^{\circ}$ illustrates the mechanism meets the design requirements of three-dimensional movement and two-dimensional rotation freedom. Due to the gap of hook joint and spherical hinge and the error of connecting link length, the motion error of moving platform is large. When the posture angle is $\alpha=0^{\circ}$ and $\beta=0^{\circ}$, the roundness error of the circle is $2 \mathrm{~mm}$, and when the posture angle is $\alpha=0^{\circ}$ and $\beta=-5^{\circ}$, the roundness error of the circle is $1.5 \mathrm{~mm}$. How to further improve the motion accuracy of the moving platform is worthy of further study in the next step.

\section{Conclusions}

(1) In this paper, based on the requirement of multidimensional 3D printing, two 5PUS-UPU parallel mechanisms with five degrees of freedom are 


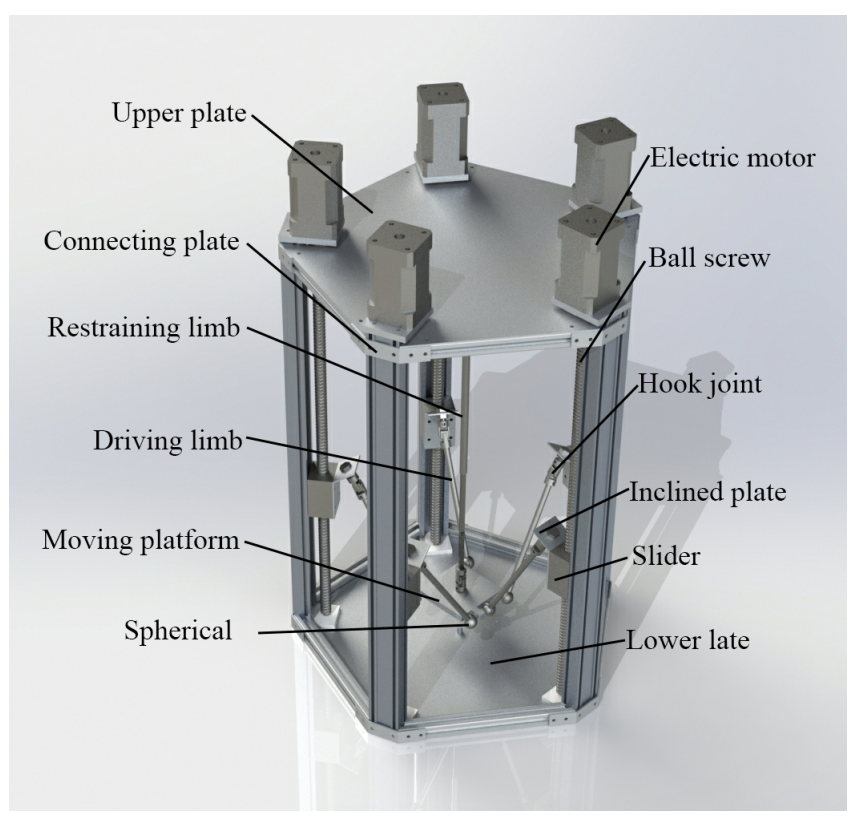

(a) 3D model of prototype

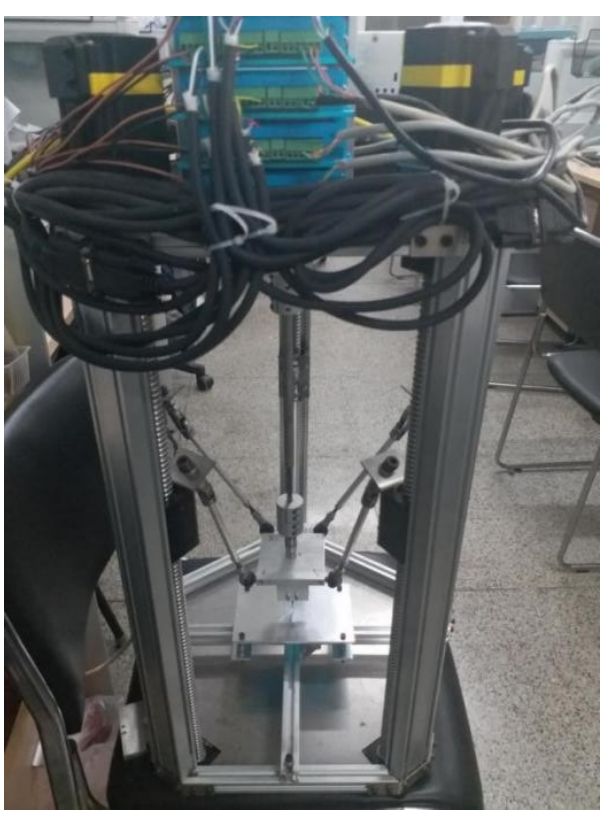

(b) Physical model of prototype

Figure 21 Prototype of parallel mechanism
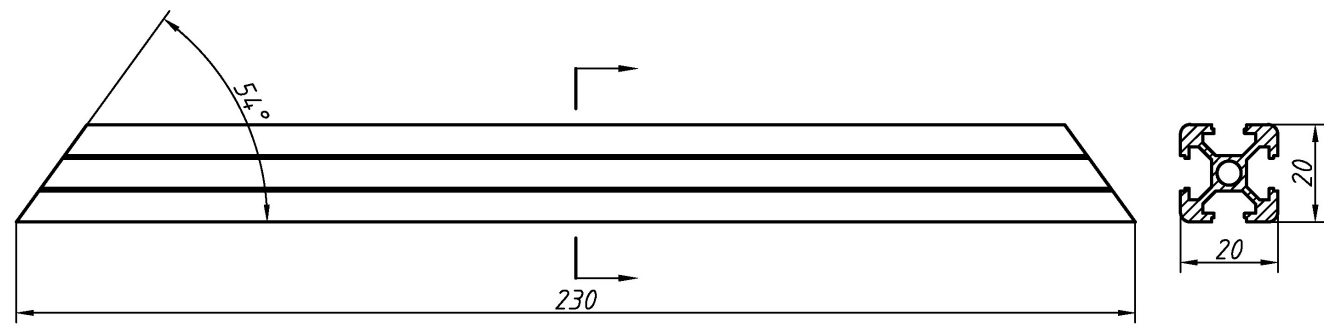

Figure 22 The size of profile 


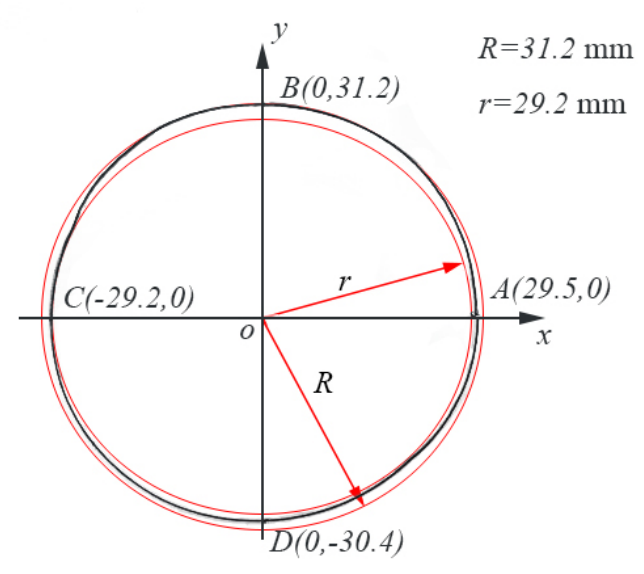

(a) $\alpha=0^{\circ}, \beta=0^{\circ}$

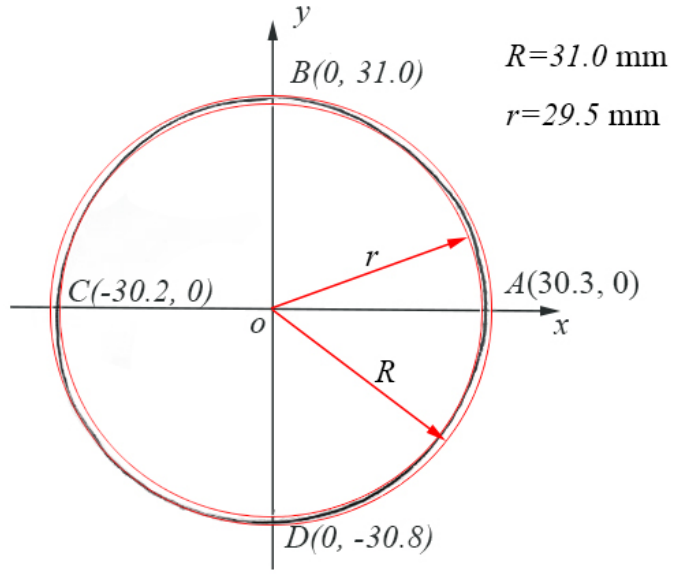

(b) $\alpha=0^{\circ}, \beta=-5^{\circ}$

Figure 23 Circular trajectories

designed, and their degrees of freedom are analyzed by using Grubler-Kutzbach and the screw theory. Based on the singularity analysis, the optimal scheme was determined in the two schemes.

(2) The coordinate system and coordinate transformation matrix are established for the optimal scheme. Based on the idea of inverse kinematics solution, the inverse position equation is established. The working condition that can reflect the 5-DOF movement of the mechanism is designed, and the position analysis, speed analysis and acceleration analysis of the working condition are carried out.

(3) The realization method of go home function is proposed. Five sliders are first searched for zero point at the same speed in the positive direction of the $Z$-axis of the fixed platform, and then stop moving after reaching their respective preset reference points. After the five sliders reach the reference point, the moving platform returns to the go home reference point. Finally, move the five sliders down a fixed distance from the reference point so that the moving platform reaches zero point.
(4) Monte Carlo method is applyed to analyze the workspace of the mechanism and to study the influencing factors of the workspace. Among the many influencing factors in the workspace, two factors are chosen: the length of the link and the position of the spherical hinge. The mechanism is optimized to obtain the optimal link length of $205 \mathrm{~mm}$ and the optimal position of the spherical hinge. It is concluded that the workspace size is $120 \mathrm{~mm}$ $\times 175 \mathrm{~mm} \times 300 \mathrm{~mm}$ when the fixed posture is $\alpha=0^{\circ}$ and $\beta=0^{\circ}$, and $80 \mathrm{~mm} \times 150 \mathrm{~mm} \times 300$ mm when $\alpha$ and $\beta$ are within $\pm 5^{\circ}$.

(5) Design the experiment, set the posture angle of the moving platform in $\alpha=0^{\circ}, \beta=0^{\circ}$ and $\alpha=$ $0^{\circ}, \beta=-5^{\circ}$, use a pencil replace the printing nozzle to draw a circle. By drawing the circular trajectory, it can be concluded that the mechanism meets the design requirements for the freedom of three-dimensional movement and two-dimensional rotation.

(6) In the experimental analysis, although the experimental results basically meet the design requirements, the kinematic accuracy of the mechanism 
is low due to the gap of hook joint and spherical hinge and the error of connecting link length. Therefore, how to further improve the motion accuracy of the mechanism will be the focus of the next work.

\section{Acknowledgements}

Not applicable

\section{Funding}

This research did not receive any specific grant from funding agencies in the public, commercial, or not-for-profit sectors.

\section{Availability of data and materials}

Not applicable

\section{Competing interests}

The authors declare that they have no competing interests.

\section{Authors' contributions}

YW conceived the basic idea, and carried out research and analysis of the manuscript. $\mathrm{CL}$ assisted with the research and wrote the manuscript. JL provided theoretical guidance. JZ contributed the revise of this paper. $Z J$ was in charge of technical expression and language. All authors read and approved the final manuscript.

\section{Author details}

School of Mechanial Engineering, University of Science and Technology Beijing, Beijing, China.

\section{References}

1. Lu B, Li D. Development of the Additive Manufacturing(3D printing)Technology. Machine Building \& Automation. 2013;42(4):1-4.

2. Wu G, Hsu S. Review: Polymeric-Based 3D Printing for Tissue Engineering. Journal of Medical \& Biological Engineering. 2015;35:285-292.

3. Vatani M, Lu Y, Engeberg EDea. Combined 3D printing technologies and material for fabrication of tactile sensors. International Journal of Precision Engineering \& Manufacturing. 2015;16(7):1375-1383.

4. Yang J, Qiu Z, Zhang X. Self-excited vibration control of a planar 3-RRR flexible parallel robot. Journal of Vibration and Shock. 2017;36(21):138-143.

5. Shan X, Cheng G. Structural error and friction compensation control of a $2(3 \mathrm{PUS}+\mathrm{S})$ parallel manipulator. Mechanism \& Machine Theory. 2018;124:92-103.

6. Zhan, Zhenhui, Zhang, Xianmin, Jian, Zhicong, et al. Error modelling and motion reliability analysis of a planar parallel manipulator with multiple uncertainties. Mechanism and Machine Theory: Dynamics of Machine Systems Gears and Power Trandmissions Robots and Manipulator Systems Computer-Aided Design Methods. 2018;124:55-72.
7. Yang J, Qiu Z, Zhang X. Kinematics modeling and analysis of 3-PRR parallel robot mechanism. Journal of Shandong University of Science and Technology (Natural Science). 2017;36(21):138-143.

8. Zhang D, Xu Y, Yao J, Hu B, Zhao Y. Kinematics, dynamics and stiffness analysis of a novel 3-DOF kinematically/actuation redundant planar parallel mechanism. Mechanism and Machine Theory 2017;116:203-219

9. Simionescu I, Ciupitu L, lonita LC. Static balancing with elastic systems of DELTA parallel robots. Mechanism \& Machine Theory. 2015;87:150-162.

10. Xiaobo G, Yan Z, Lina WU. Workspace Analysis of Mechanism of a 3D Printing Parallel Robotic Manipulator. Machine Tool \& Hydraulics. 2018;46(5):6-8

11. Chang-Fei BI. The Kinematic Analysis and Simulation of 3D Print Parallel Robot Mechanism Based on RecurDyn. Machinery Design \& Manufacture. 2016;(3):243-246.

12. Keating S, Oxman N. Compound fabrication: A multi-functional robotic platform for digital design and fabrication. Robotics and Computer Integrated Manufacturing. 2013;29(6):439-448.

13. Song X, Pan Y, Chen Y. Development of a Low-Cost Parallel Kinematic Machine for Multidirectional Additive Manufacturing. Journal of Manufacturing ence \& Engineering. 2015;137(2):021005.

14. Dingyong C, Yuefa F, Wei YE. Design and Analysis of a Double Output 3D Printing Decoupling Parallel Manipulator. Journal of Mechanical Engineering. 2017;53(7):39-46.

15. Ye W, Fang Y, Guo S. Design and analysis of a reconfigurable parallel mechanism for multidirectional additive manufacturing. Mechanism and Machine Theory. 2017;112:307 - 326.

16. Ying P, Yuefa F, Congzhe W. Design and Analysis of Five DOF 3D Printing Parallel Robot. China Mechanical Engineering. 2016;27(17):2273-2279.

17. Wang J, Gosselin CM. Singularity Loci of a Special Class of Spherical 3-DOF Parallel Mechanisms With Prismatic Actuators. Asme Journal of Mechanical Design. 2004;126(2):319-326.

18. Gallardo-Alvarado J, Rico-Martínez JM, Alici G. Kinematics and singularity analyses of a 4-dof parallel manipulator using screw theory. Mechanism \& Machine Theory. 2006;41(9):1048-1061

19. Guo $S$, Wang $C$, Qu H, Fang Y. A novel 4-RRCR parallel mechanism based on screw theory and its kinematics analysis. Proceedings of Institution of Mechanical Engineers Part C Journal of Mechanical Engineering ence. 2013;227(9):2039-2048.

20. Thomas MJ, Joy ML, Sudheer AP. Kinematic and Dynamic Analysis of a 3-PRUS Spatial Parallel Manipulator. Chinese Journal of Mechanical Engineering. 2020;33(1)

21. Wolf A, Ottaviano E, Shoham M, Ceccarelli M. Application of line geometry and linear complex approximation to singularity analysis of the 3-DOF CaPaMan parallel manipulator. Mechanism \& Machine Theory. 2004;39(1):75-95.

22. Tian C, Fang Y, Ge" QJ. Design and analysis of a partially decoupled generalized parallel mechanism for 3T1R motion. Mechanism and Machine Theory. 2019;140:211 - 232. 
23. Tao Z, An Q. Interference analysis and workspace optimization of 3-RRR spherical parallel mechanism. Mechanism and Machine Theory. 2013;69(6):62-72.

24. Wang L, Xu H, Guan L. Optimal design of a 3-PUU parallel mechanism with 2R1T DOFs. Mechanism and Machine Theory. 2017;114:190 - 203.

25. Bai Z, Han X, Chen W. Optimal design of parallel mechanisms with large tilting ability based on redundant actuation. Journal of Beijing University of Aeronautics and Astronautics. 2006;(07):856-859.

26. Stewart, D. A Platform with Six Degrees of Freedom. ARCHIVE Proceedings of the Institution of Mechanical Engineers 1847-1982 (vols 1-196). 1965;180(1965):371-386.

27. Ye Z, Zhihui H, Liping T. Research on Structure Synthesis of Five Degrees of Freedoms Hybrid Mechanisms. Machine Tool \& Hydraulics. 2016;44(17):43-48.

28. Yiqun Z, Junchuan N. Coordinates-reduction Method of Parallel Mechanism based on Screw Theory. Mechanical ence and Technology for Aerospace Engineering. 2017;(05):1-5.

29. Huang Z. Theory and Control of Parallel Robot Mechanism. Beijing: China Machine Press; 1997.

30. Yan W. Dynamics Analysis of A Novel Re-configurable Double-tripod hybrid robot. East China Jiaotong University; 2011.

31. Cheng G, Gu W, Jiang S. Singularity Analysis of a Parallel Hip Joint Simulator Based on Grassmann Line Geometry. Journal of Mechanical Engineering. 2012;48(17):29-37.

32. Li S, Liu M, Zhang Y. Research on Screw System Dependency under Different Space Geometrical Conditions. China Mechanical Engineering. 2007;18(6):655-658.

33. Cao Y. On Singular Configurations of Six Degrees of Freedom of Parallel Manipulators. Yanshan University; 2006. 
Figures

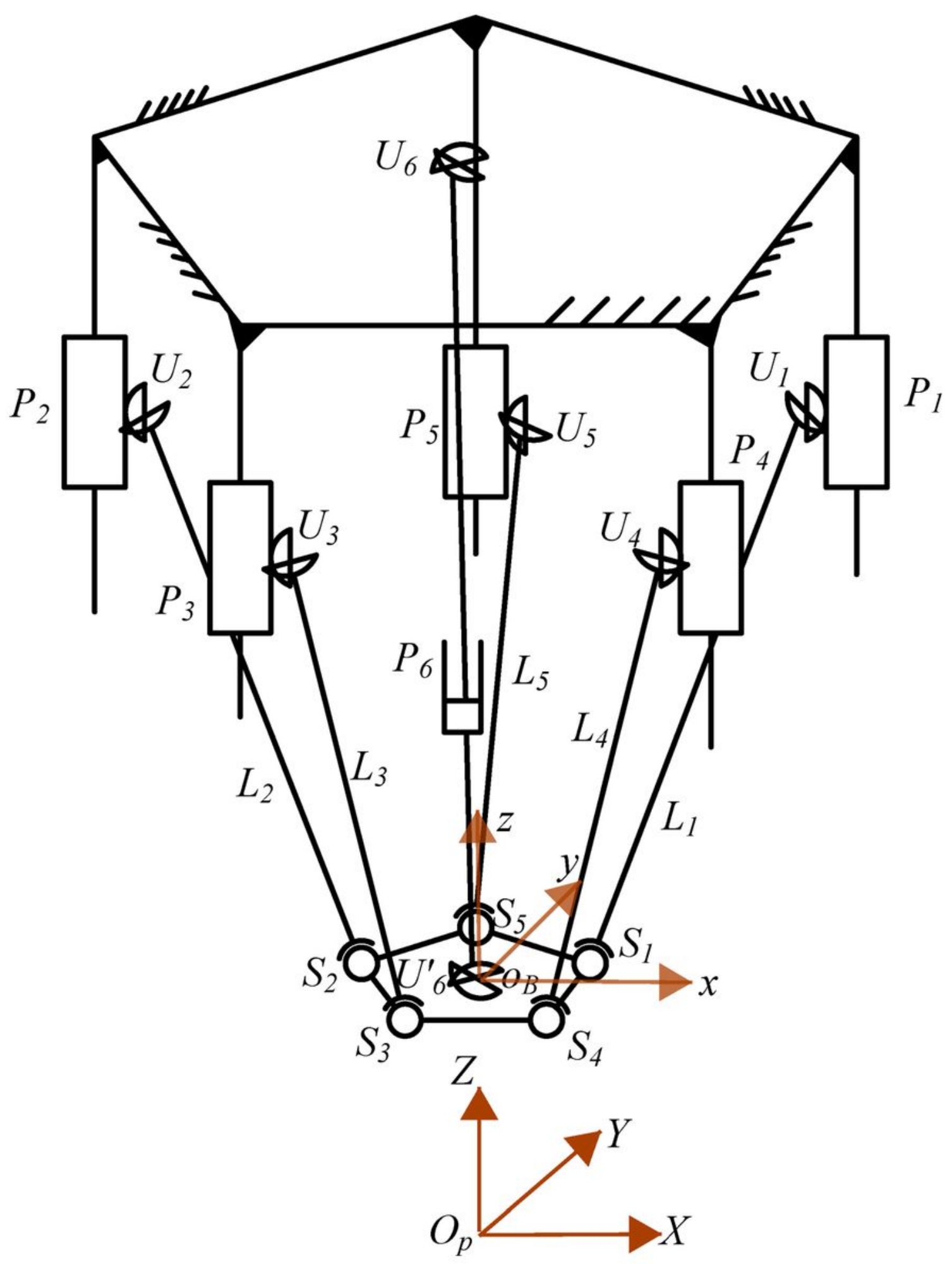

Figure 1

Design sketch of parallel mechanism 


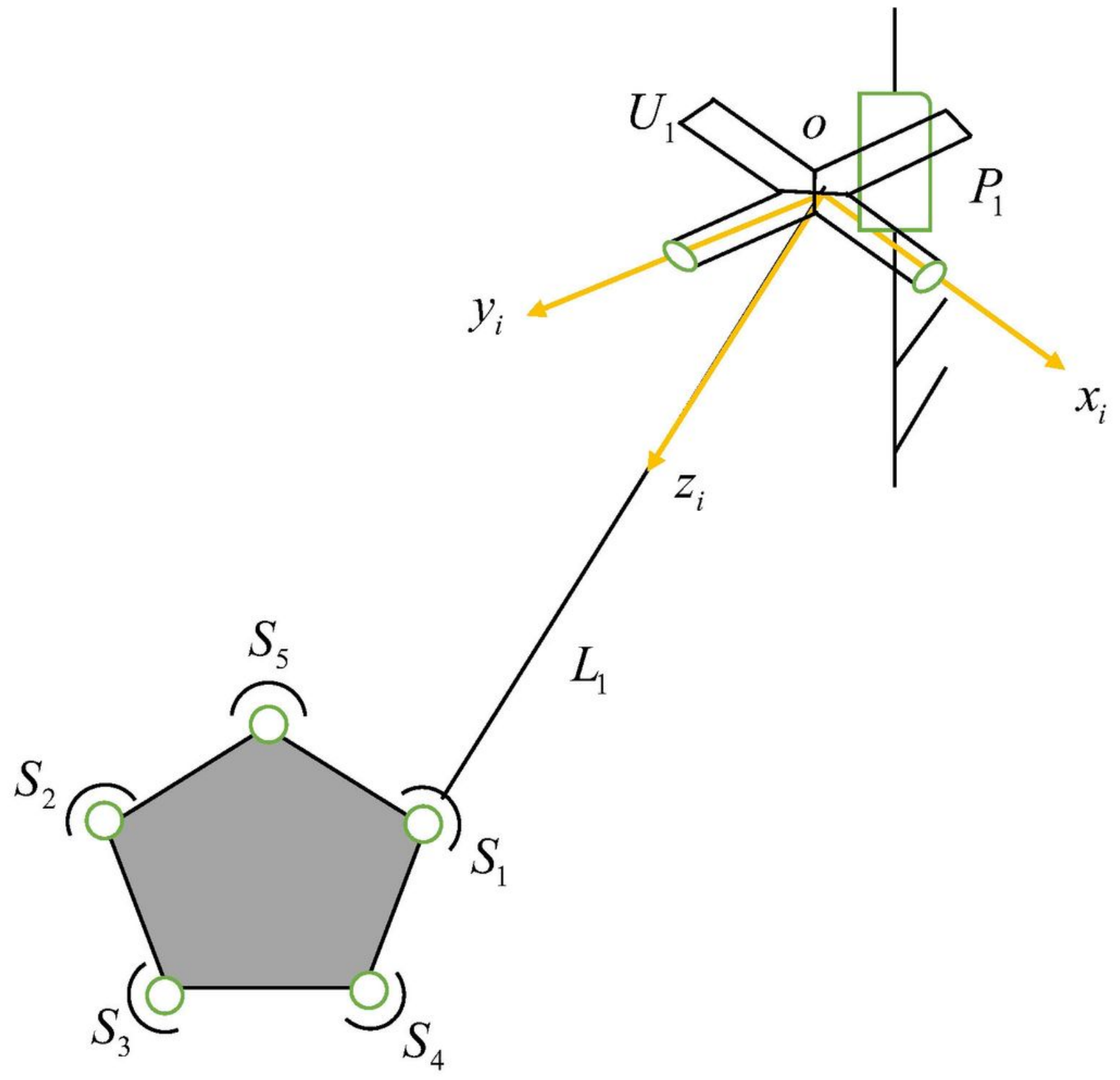

Figure 2

Sketch of limb 1 


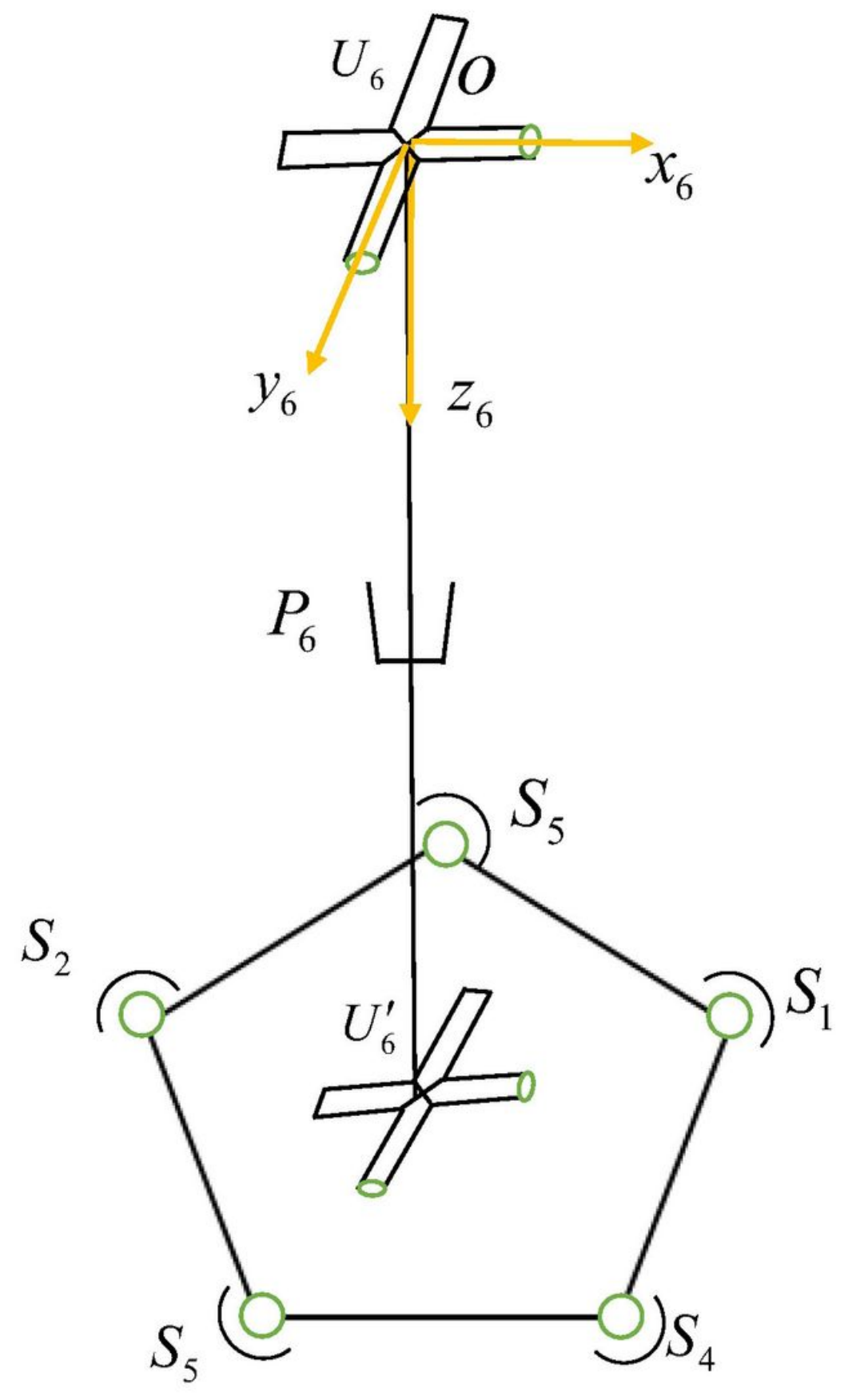

Figure 3

Sketch of limb 6 


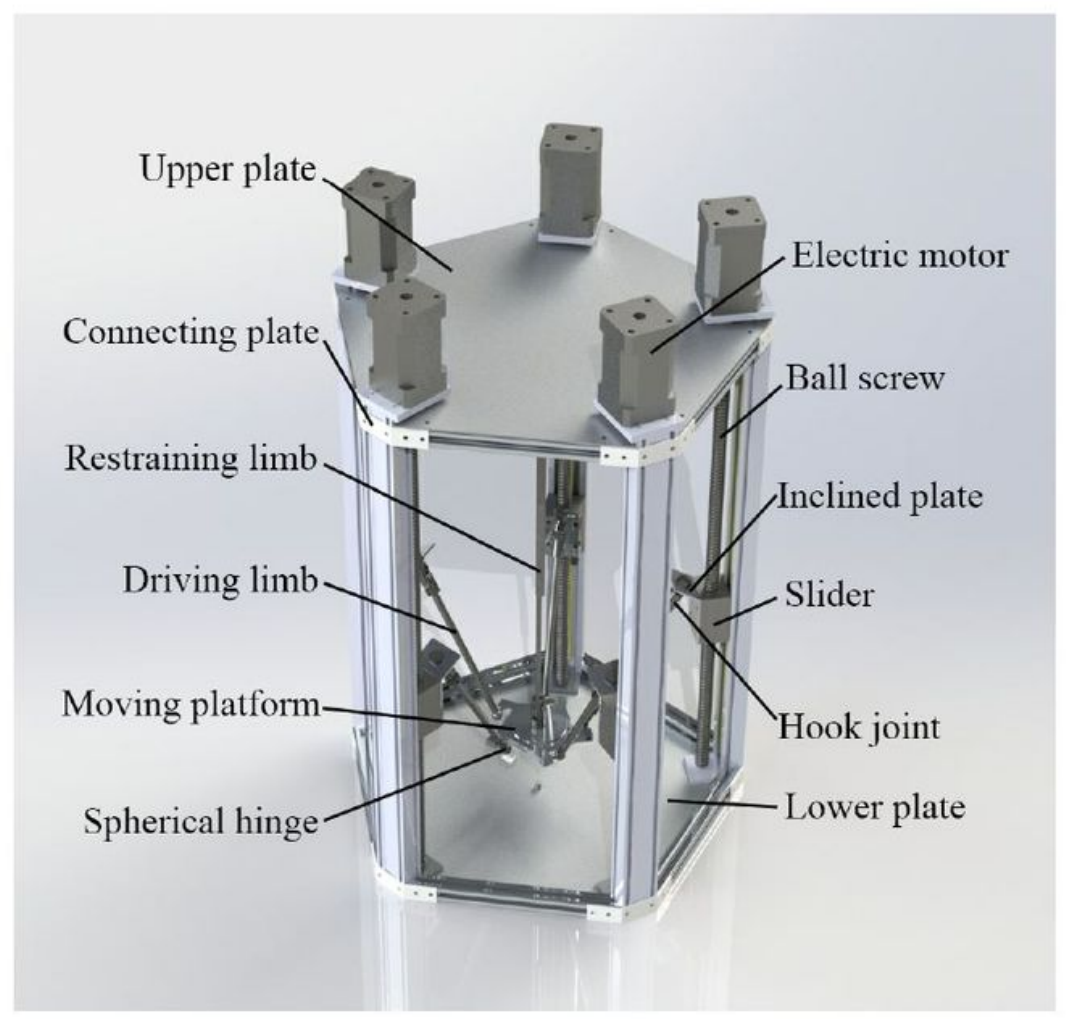

(a) three-dimensional model

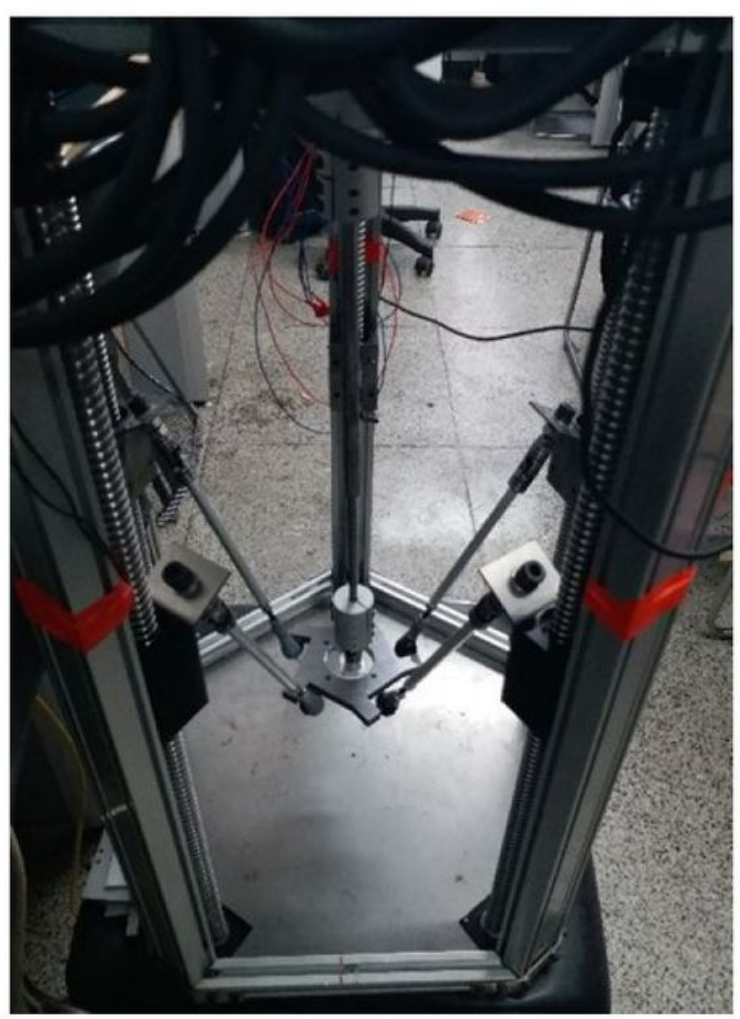

(b) physical prototype

Figure 4

Three-dimensional model and physical prototype 


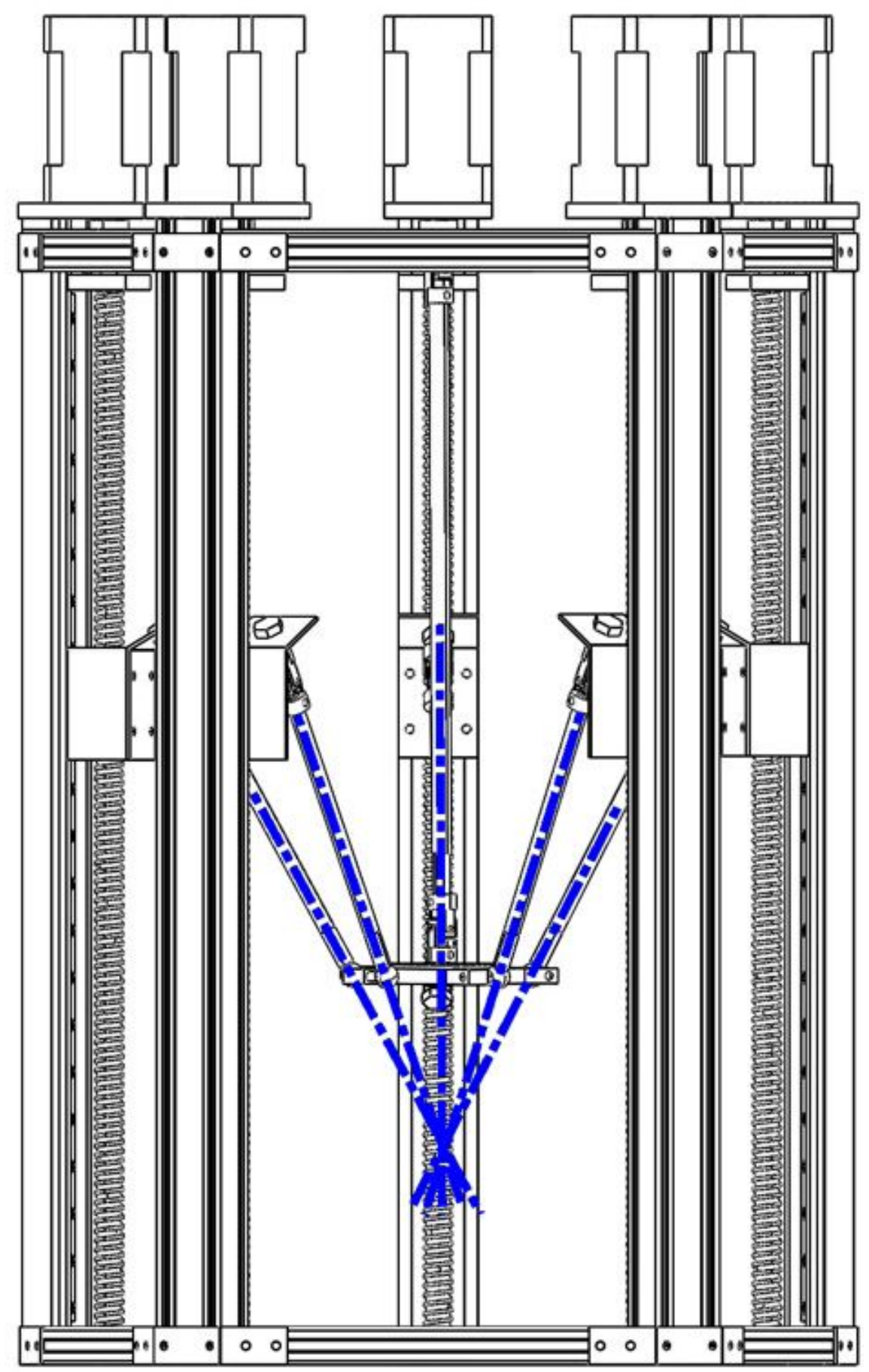

Figure 5

Singular position of preliminary scheme 


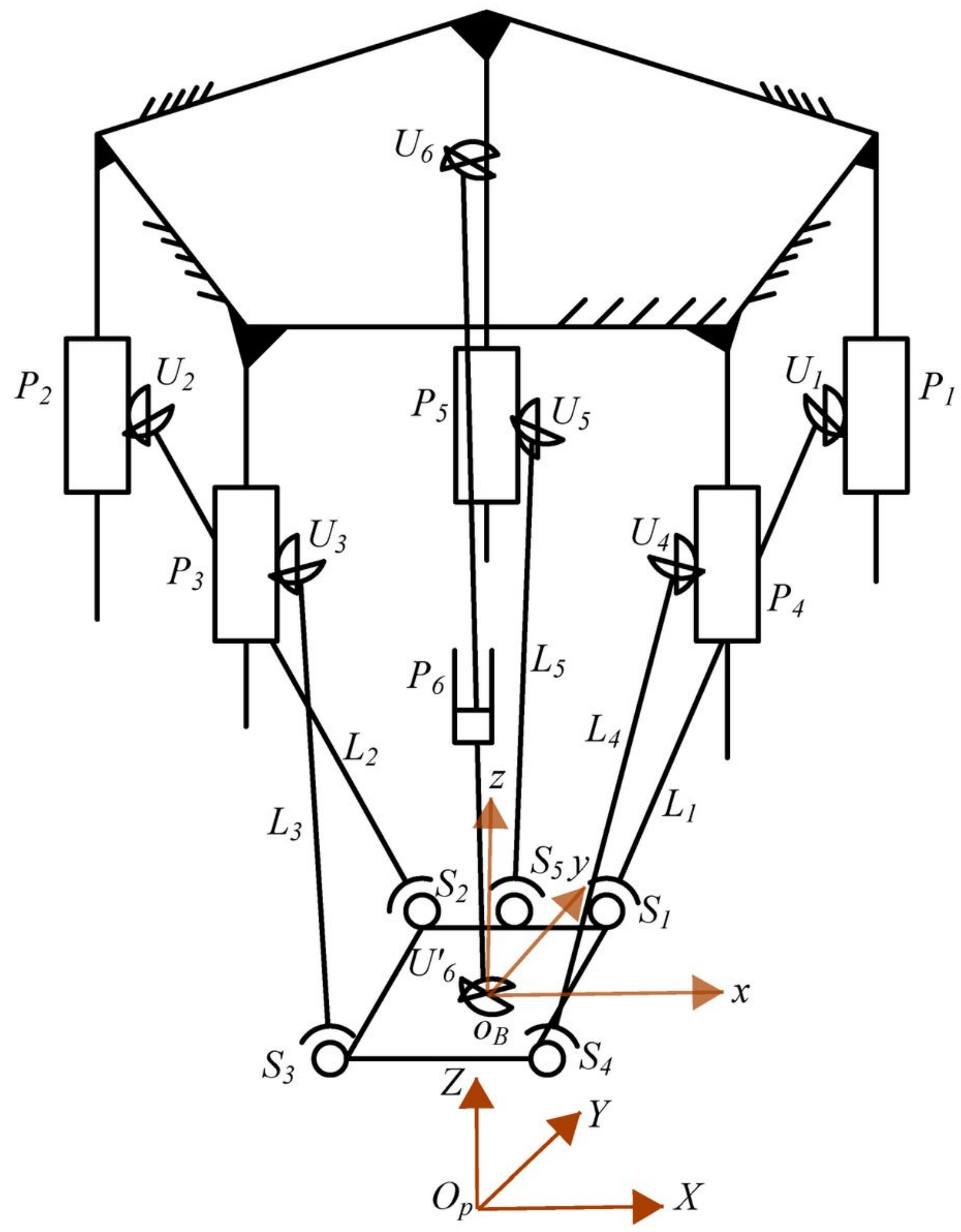

Figure 6

Mechanism schematic diagram of the improved scheme 


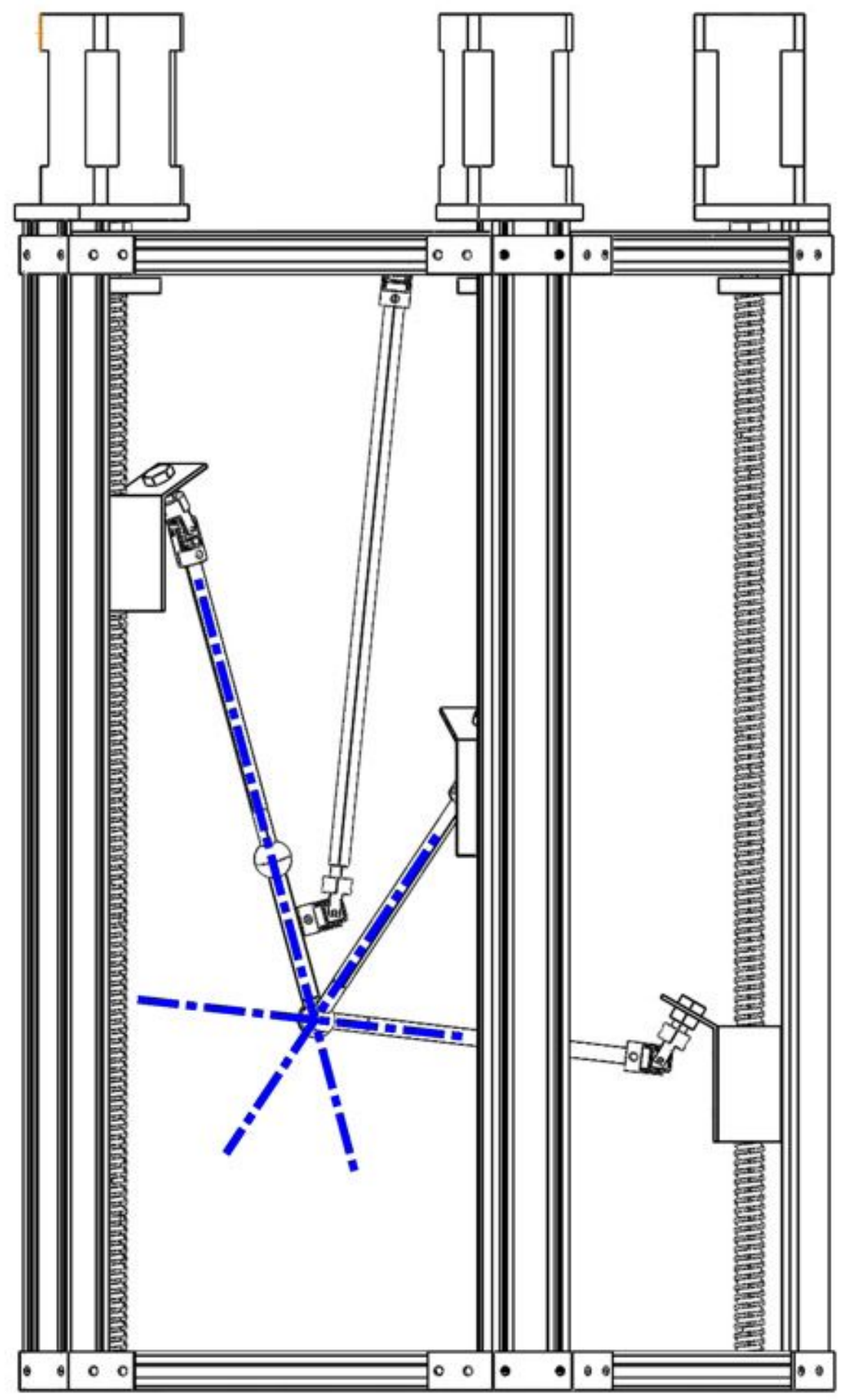

Figure 7

Singularity of improved scheme 


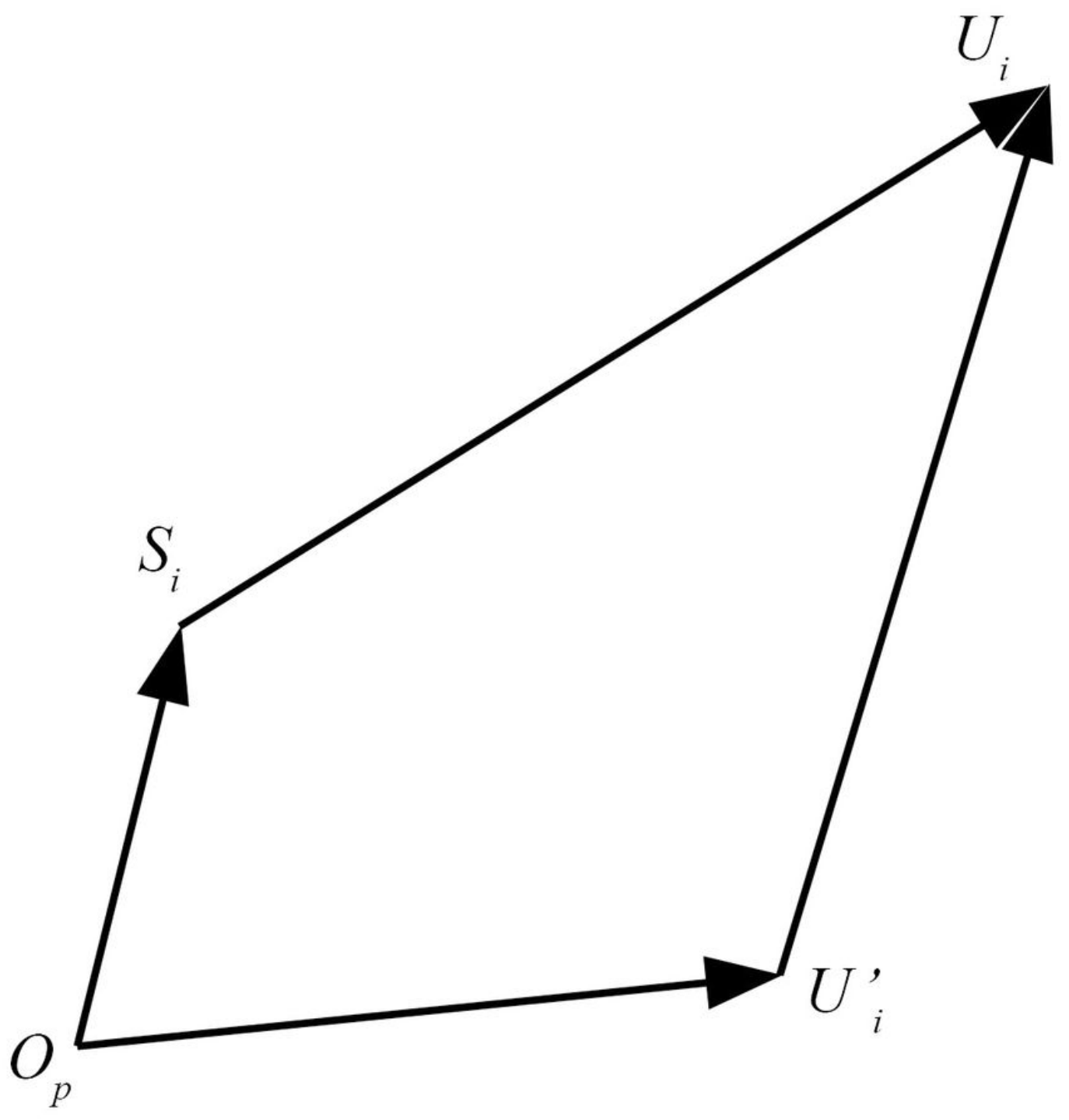

Figure 8

Closed-loop vector diagram 


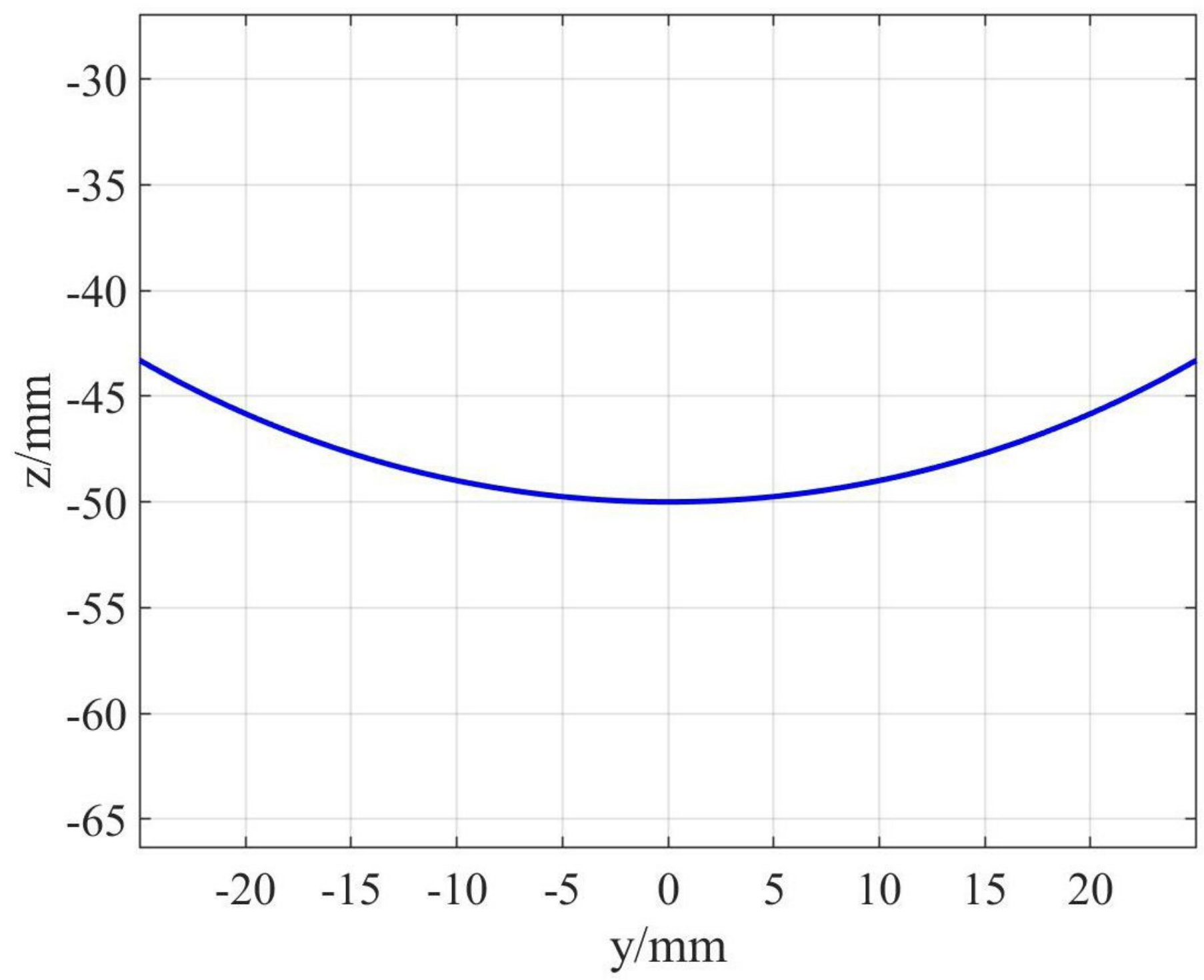

Figure 9

The motion path of the moving platform 


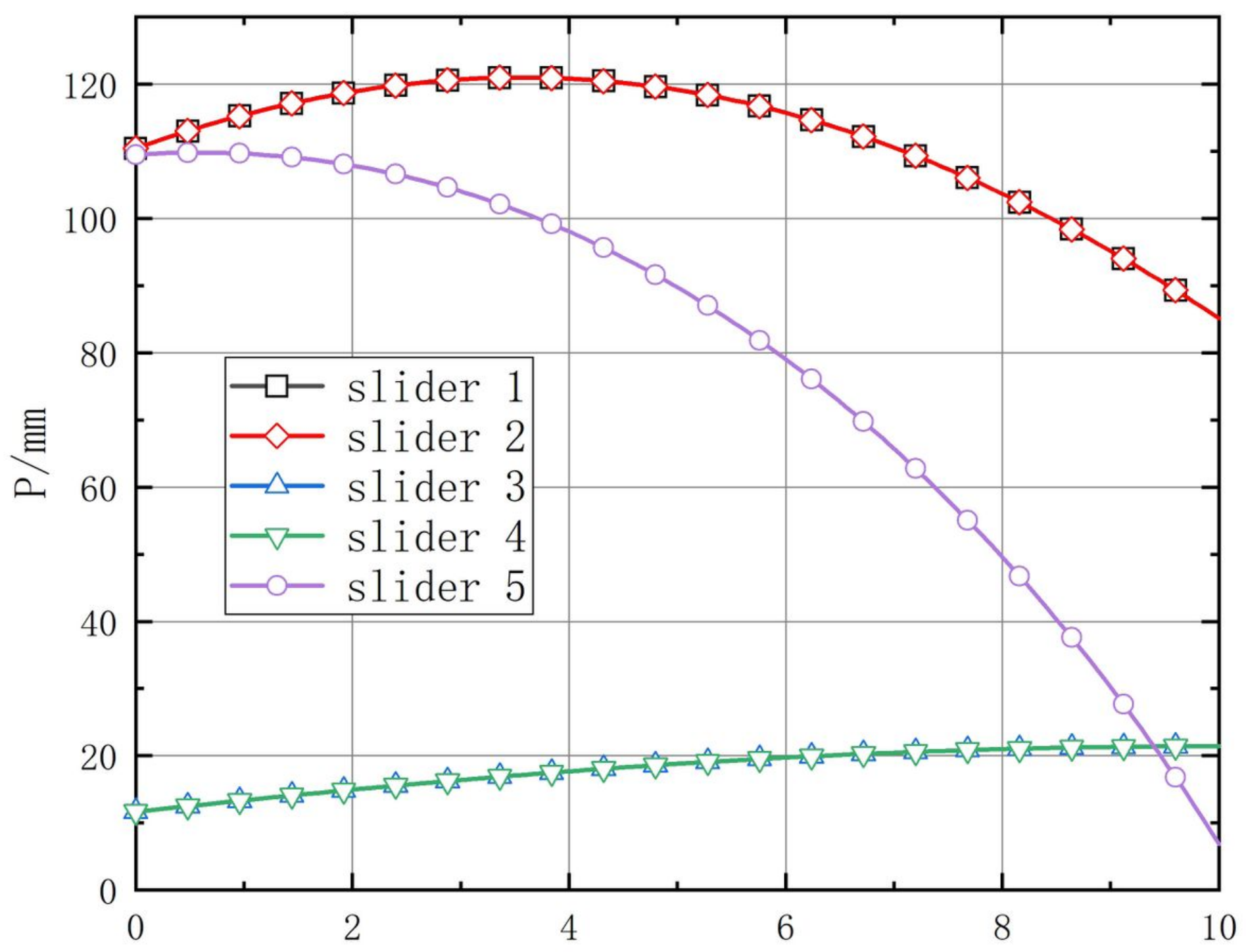

Figure 10

Position curves of sliders 


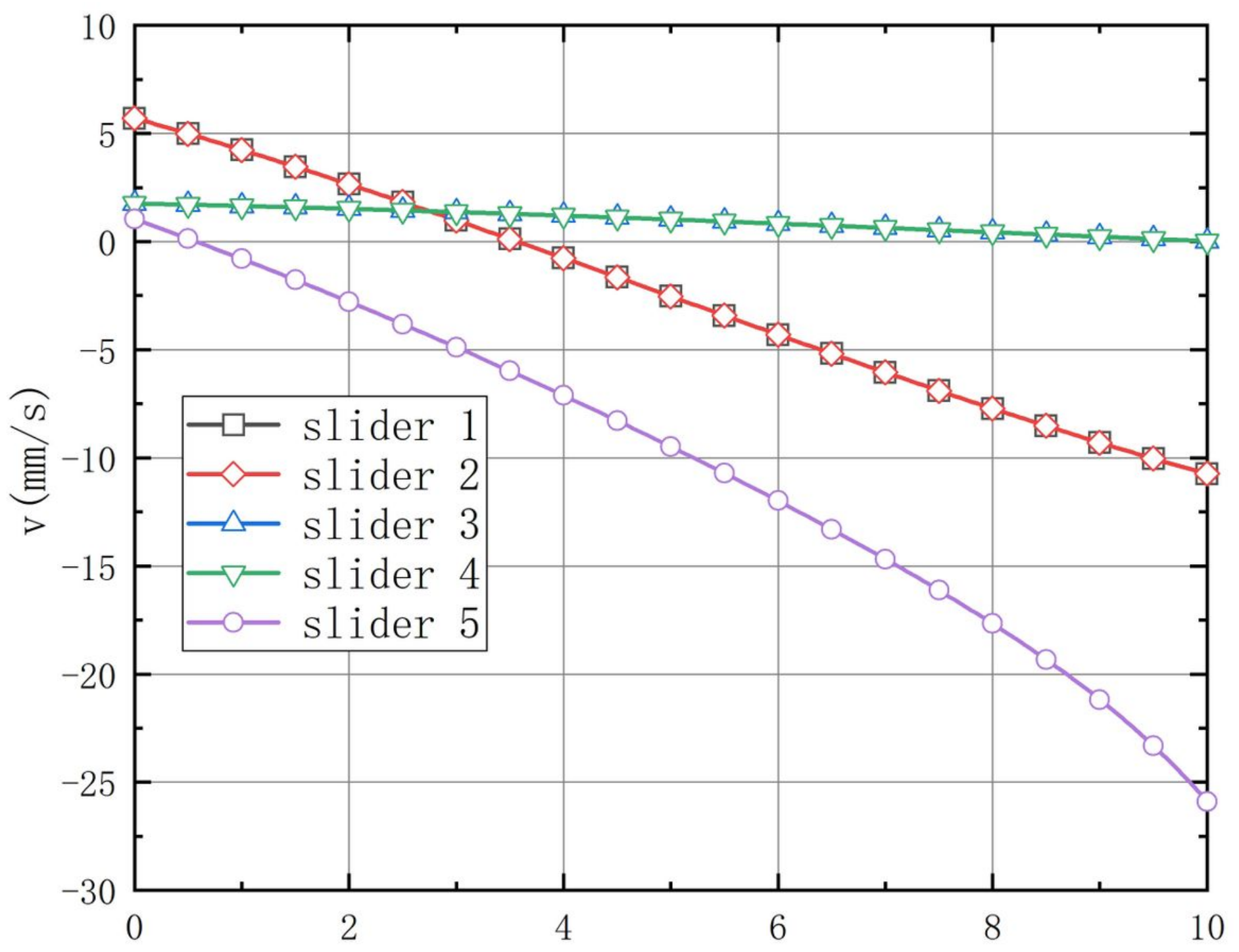

Figure 11

Velocity curves of sliders 


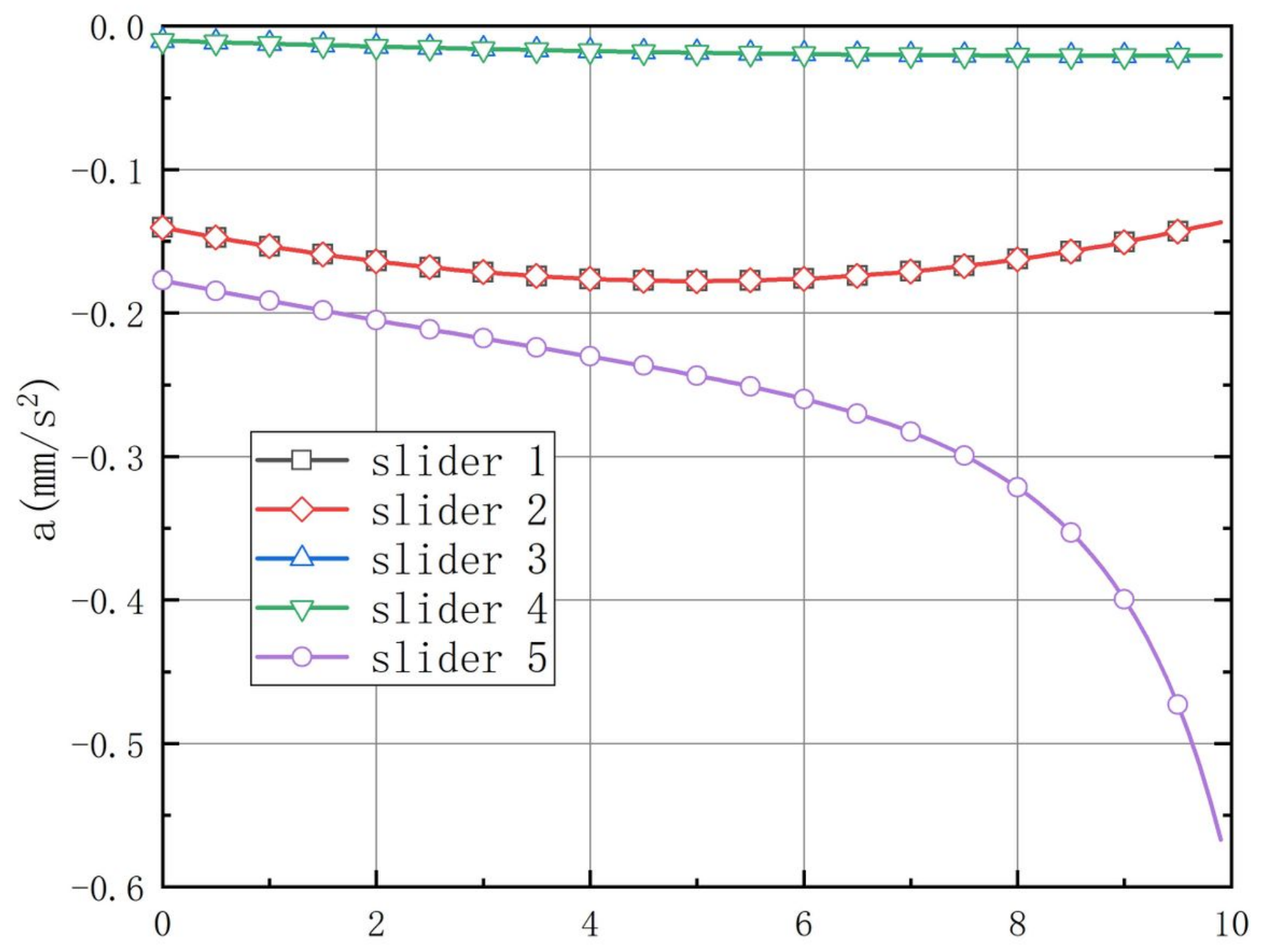

Figure 12

Acceleration curves of sliders

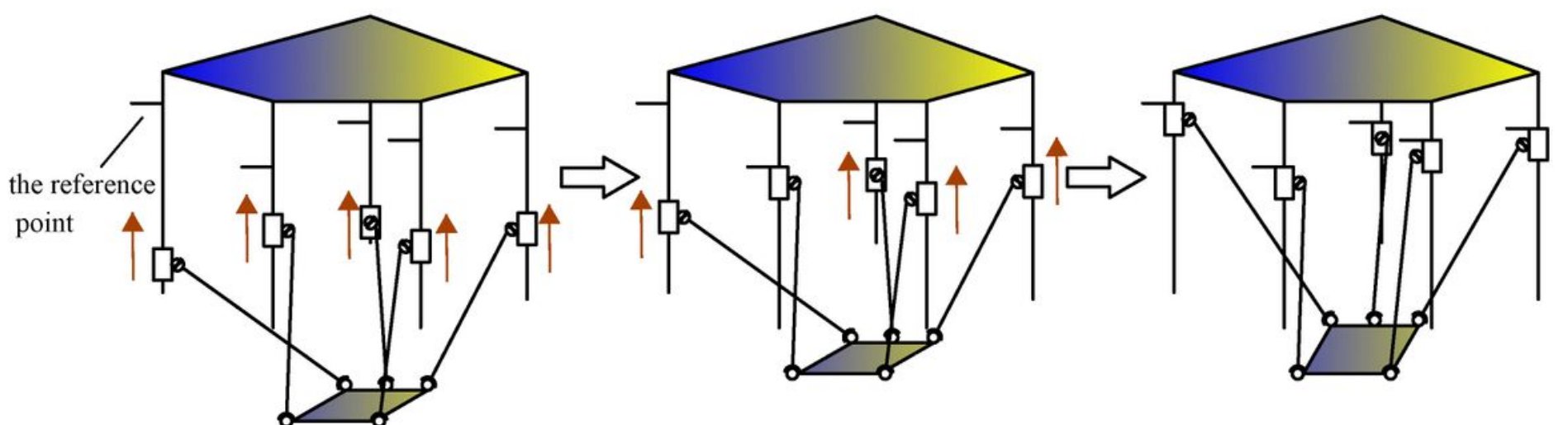

(1) Five sliders move up at the same speed. (2) Slider that first reaches the reference point stops moving.

(3) When all sliders reach the reference point, the mechanism returns to the zero reference point. 


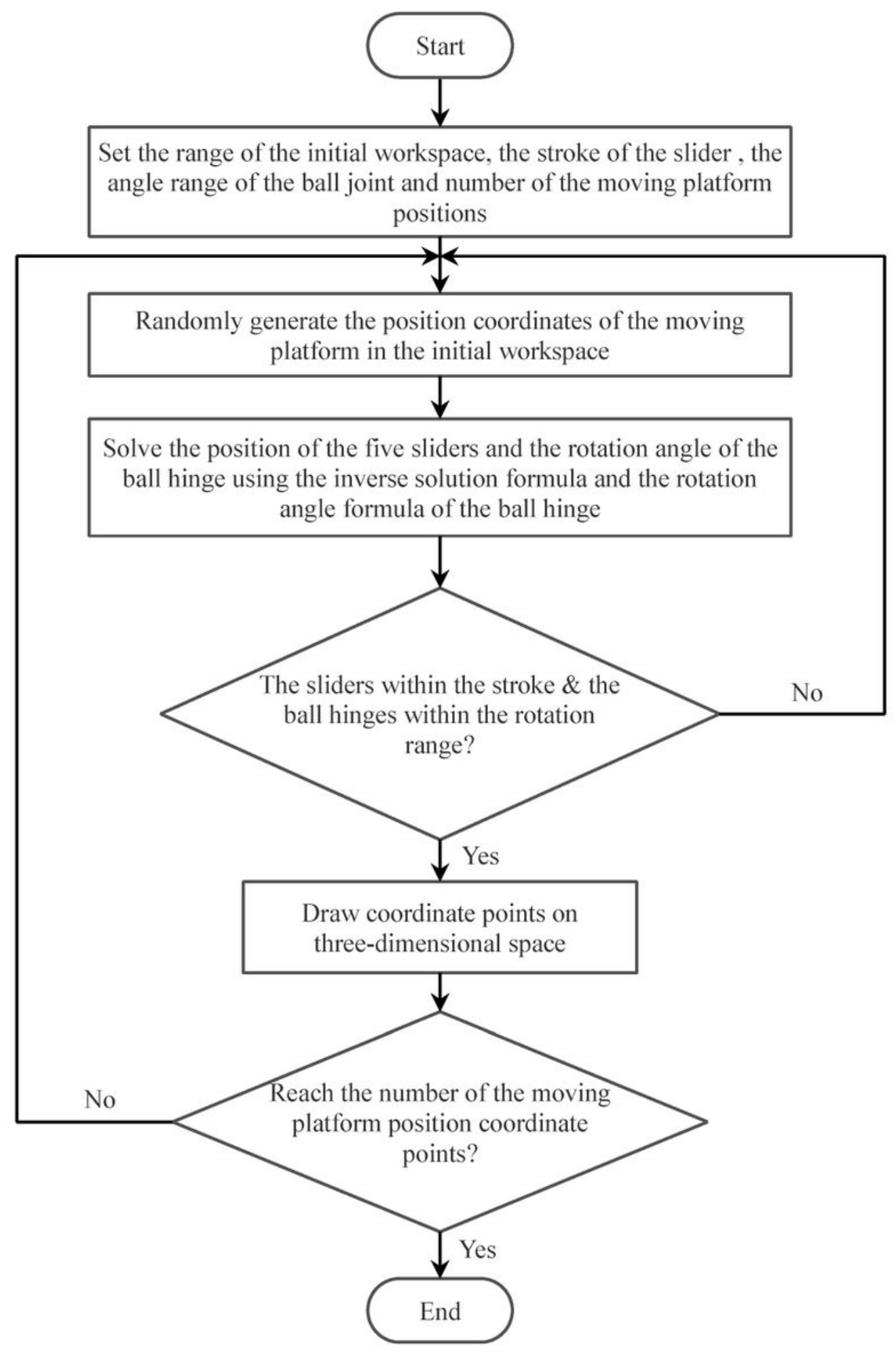

Figure 14

The flowchart of solving mechanism workspace by Monte Carlo method 


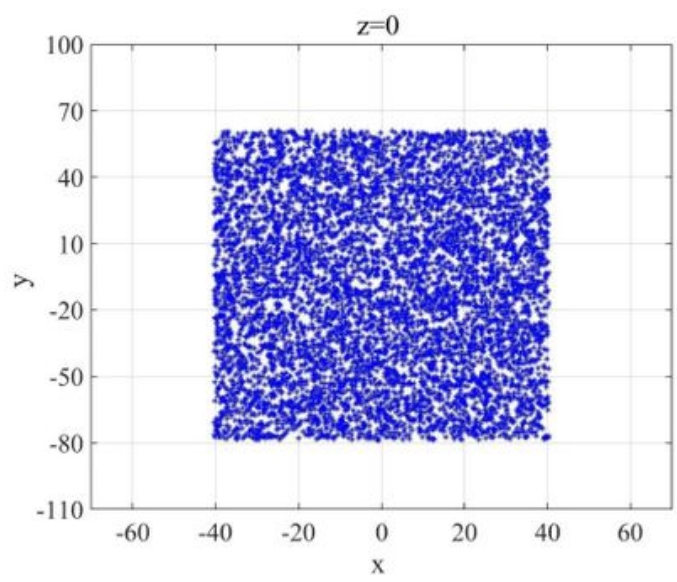

(a) $L=165 \mathrm{~mm}, Z=0 \mathrm{~mm}$

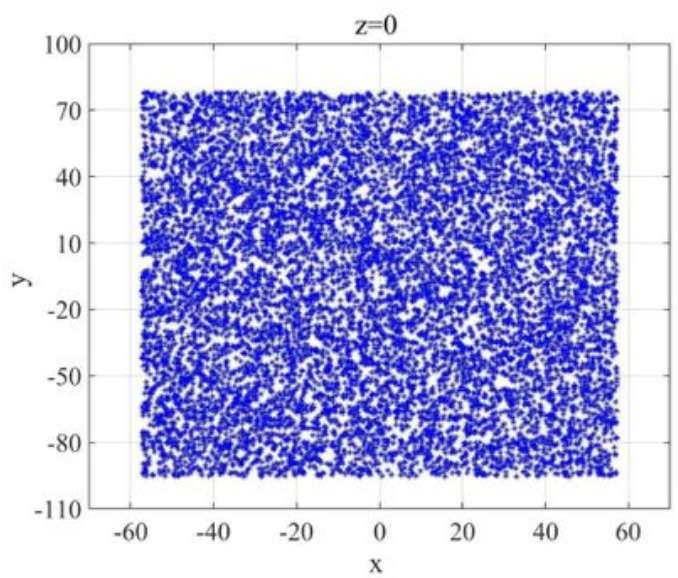

(c) $L=205 \mathrm{~mm}, Z=0 \mathrm{~mm}$

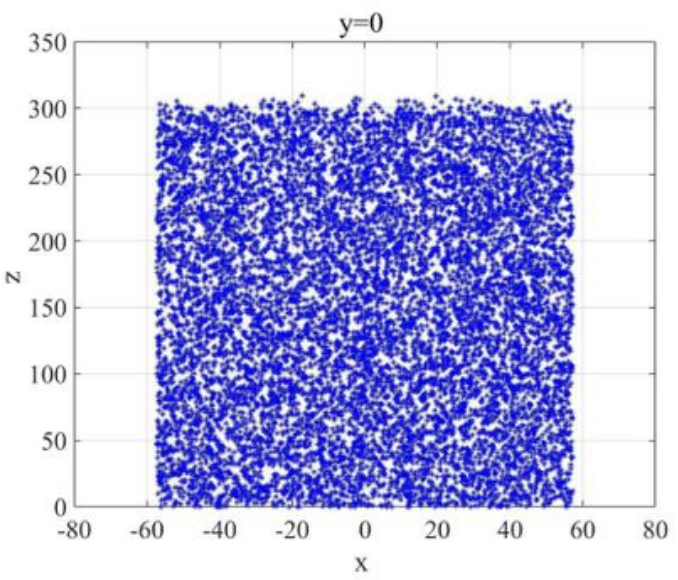

(e) $L=205 \mathrm{~mm}, Y=0 \mathrm{~mm}$

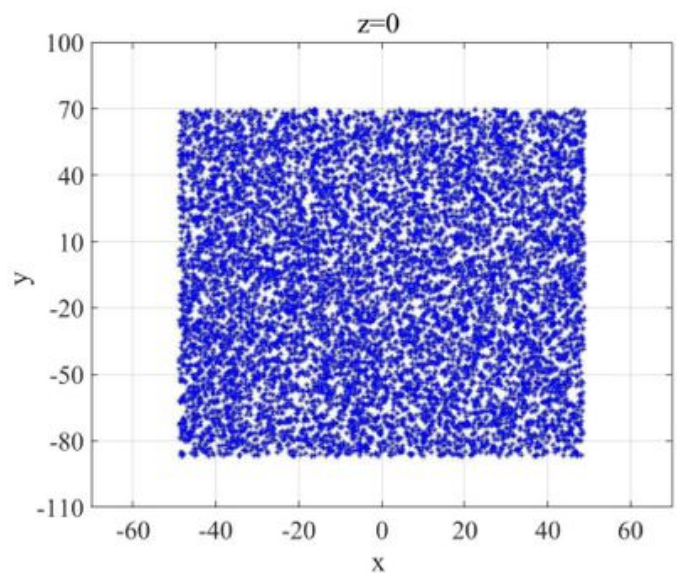

(b) $L=185 \mathrm{~mm}, Z=0 \mathrm{~mm}$

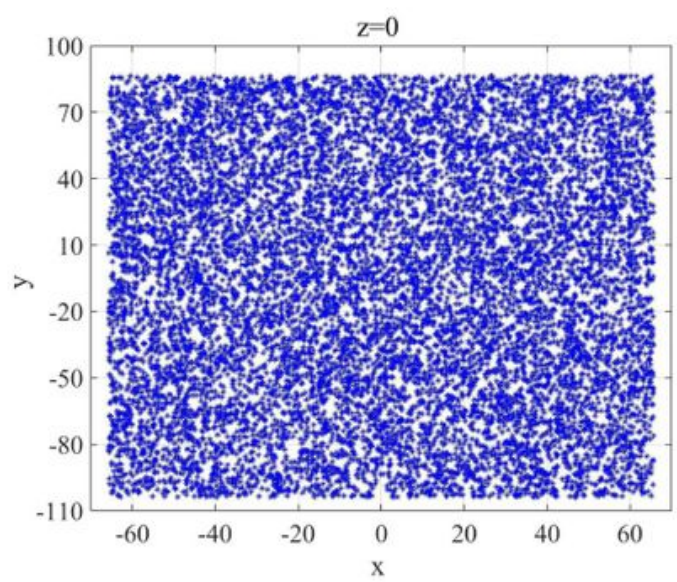

(d) $L=225 \mathrm{~mm}, Z=0 \mathrm{~mm}$

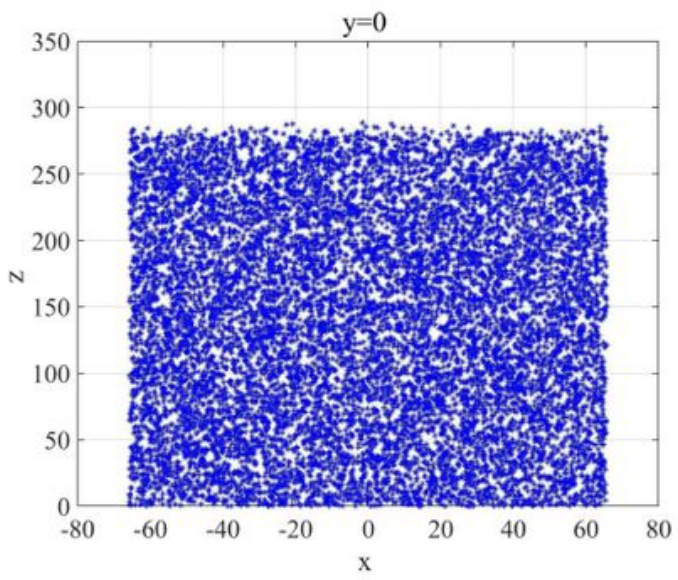

(f) $L=225 \mathrm{~mm}, Y=0 \mathrm{~mm}$

\section{Figure 15}

Limitation of link length interference 


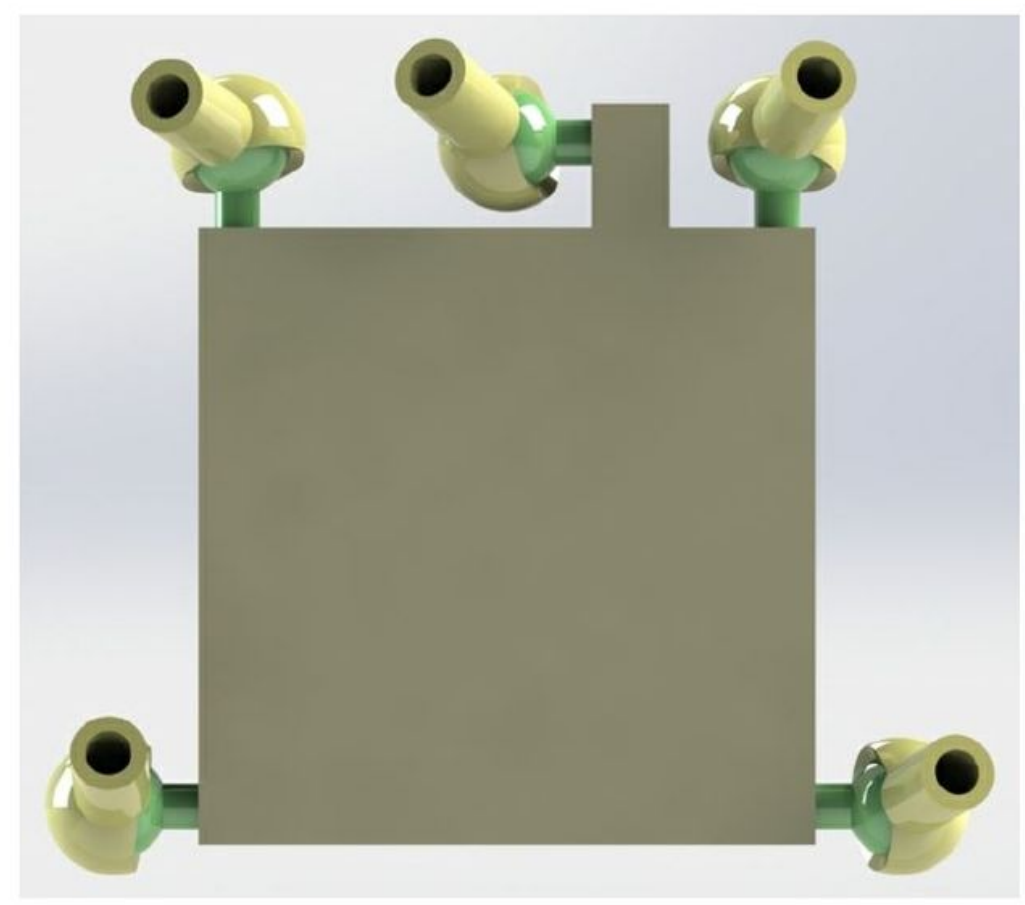

(a) Scheme 1

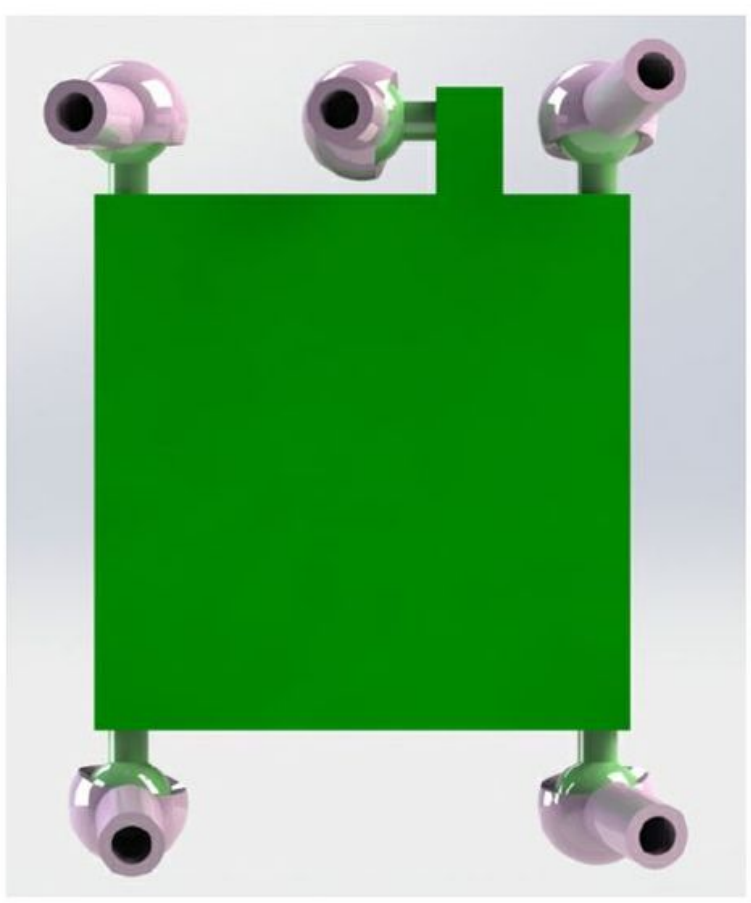

(b) Scheme 2

\section{Figure 16}

Distribution of spherical hinges on the moving platform

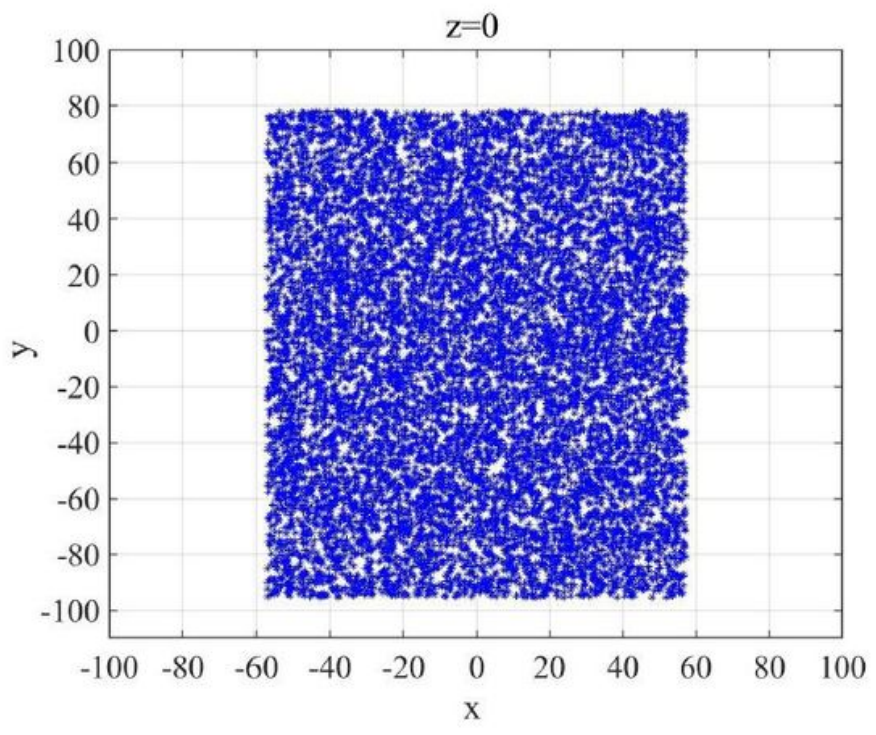

(a) Scheme 1

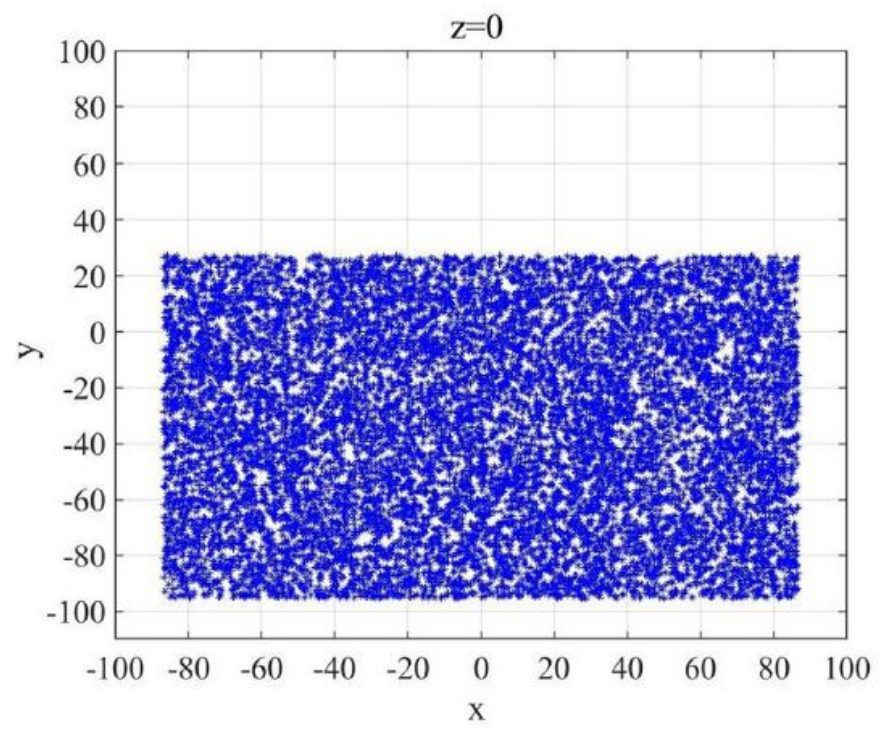

(b) Scheme 2

\section{Figure 17}

Workspace diagrams of the distribution of spherical hinges for schemes 1 and 2 


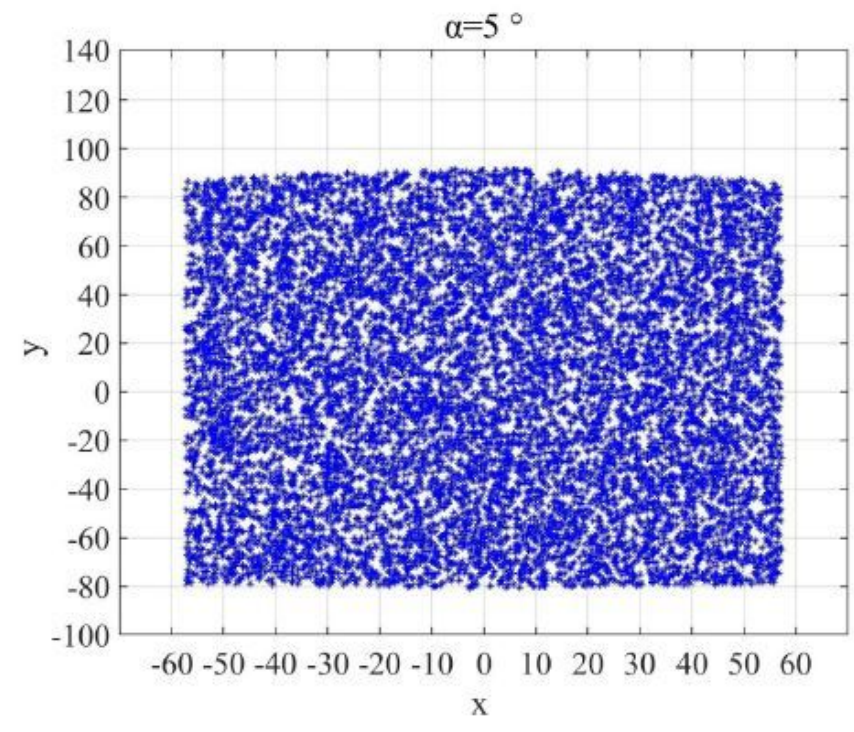

(a) $\alpha=5^{\circ}$

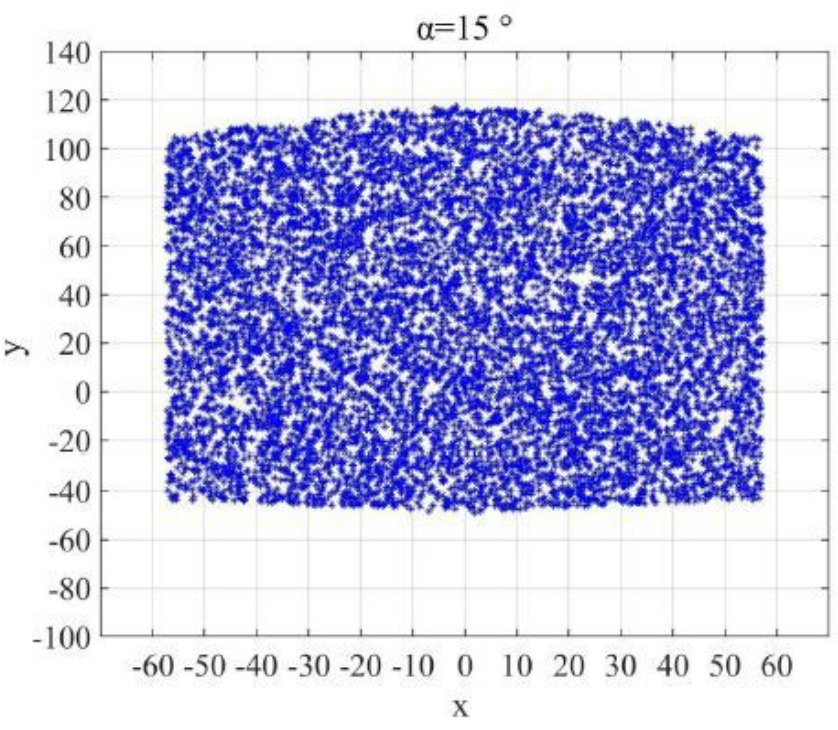

(c) $\alpha=15^{\circ}$

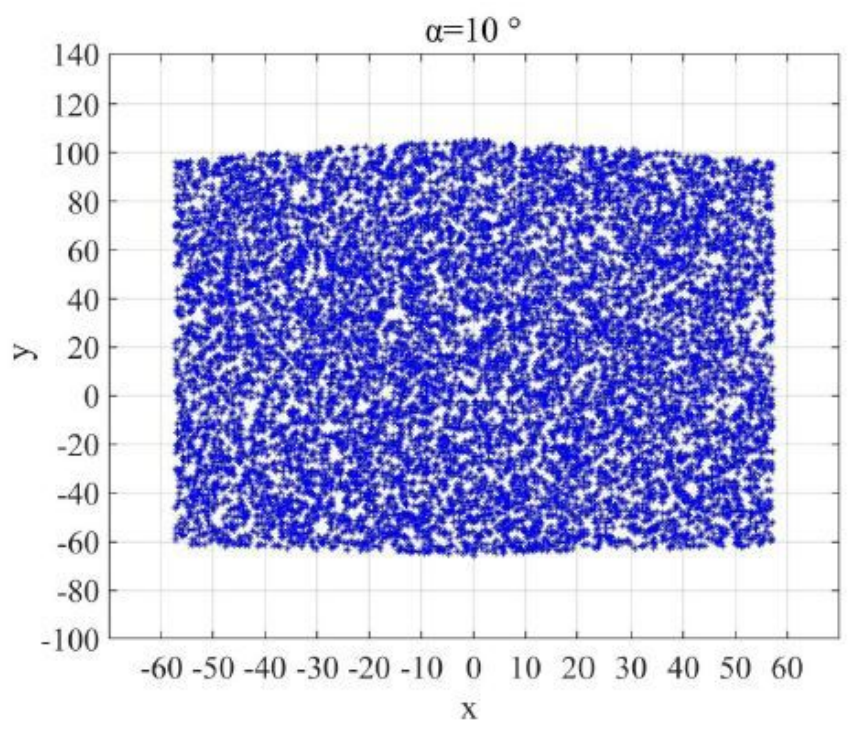

(b) $\alpha=10^{\circ}$

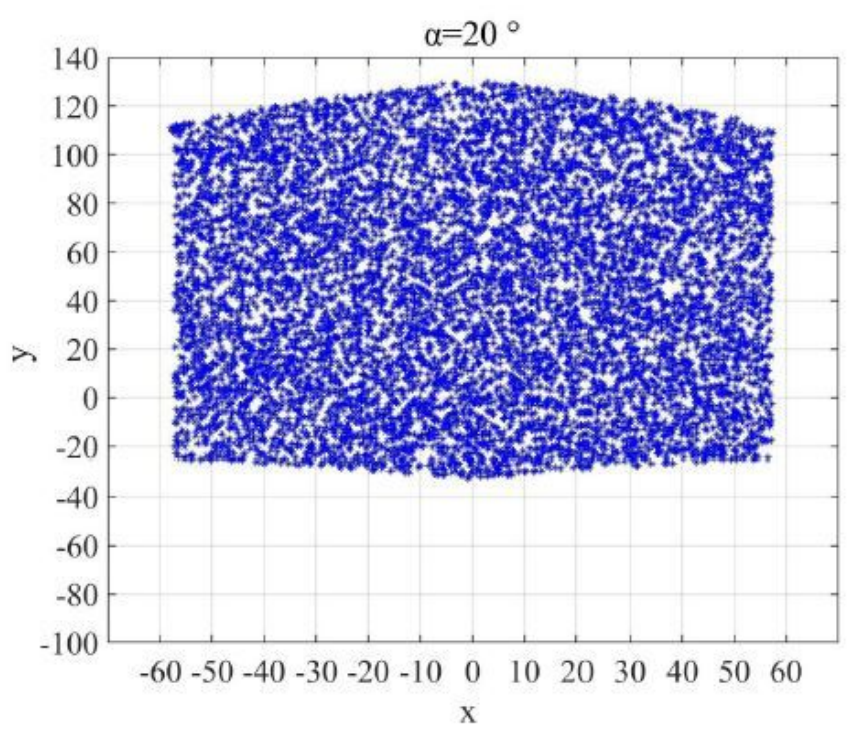

(d) $\alpha=20^{\circ}$

\section{Figure 18}

Fixed posture workspace of a 


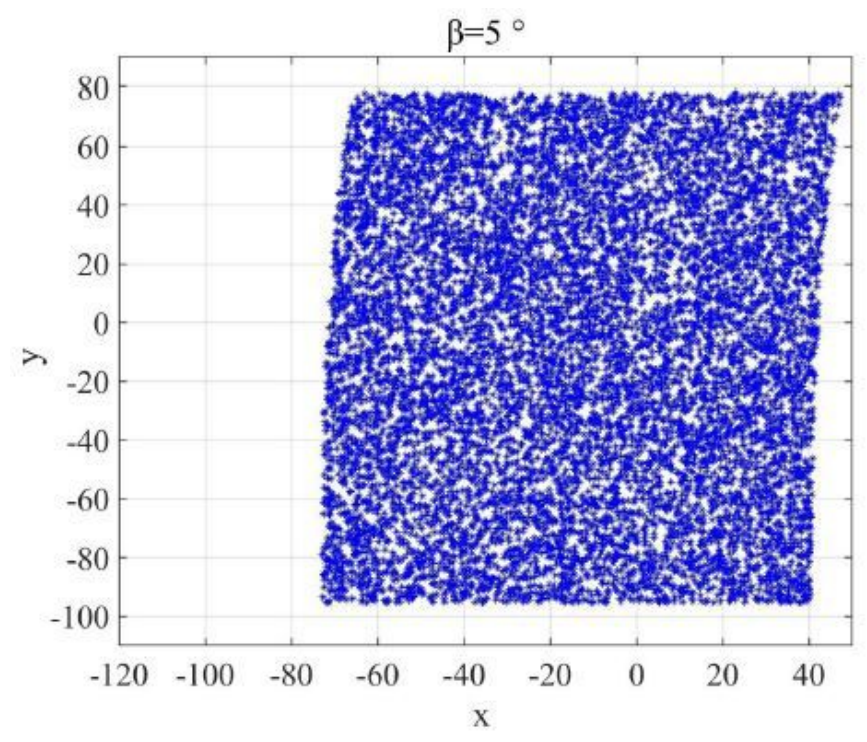

(a) $\beta=5^{\circ}$

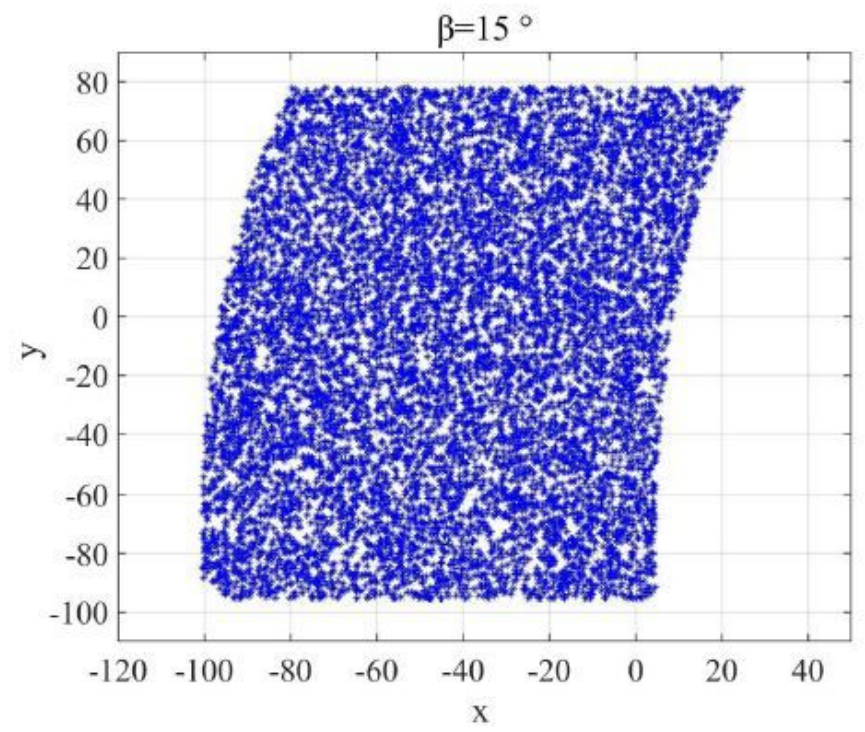

(c) $\beta=15^{\circ}$

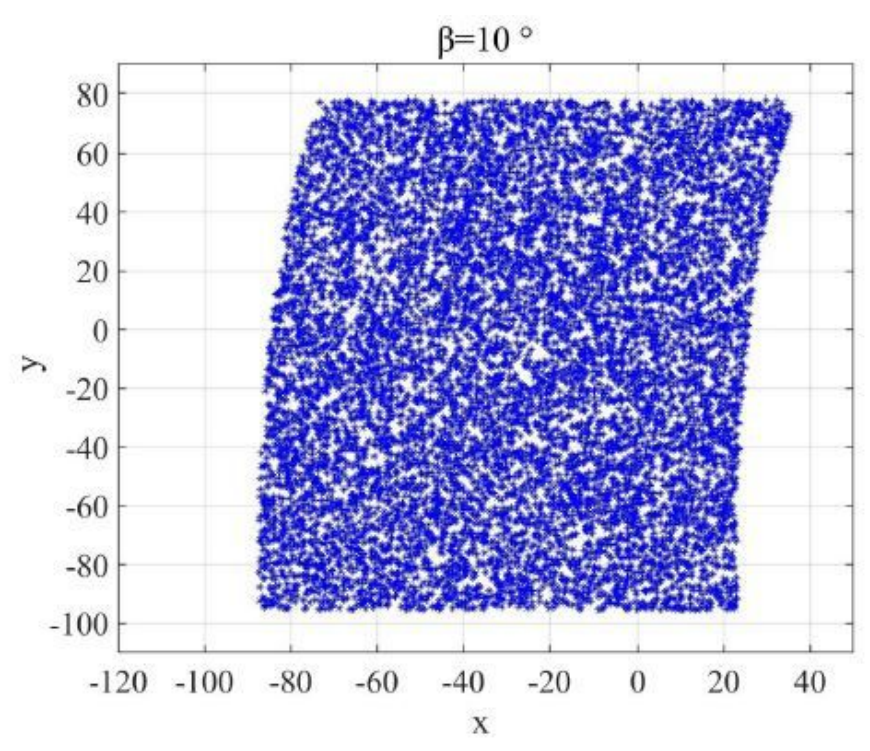

(b) $\beta=10^{\circ}$

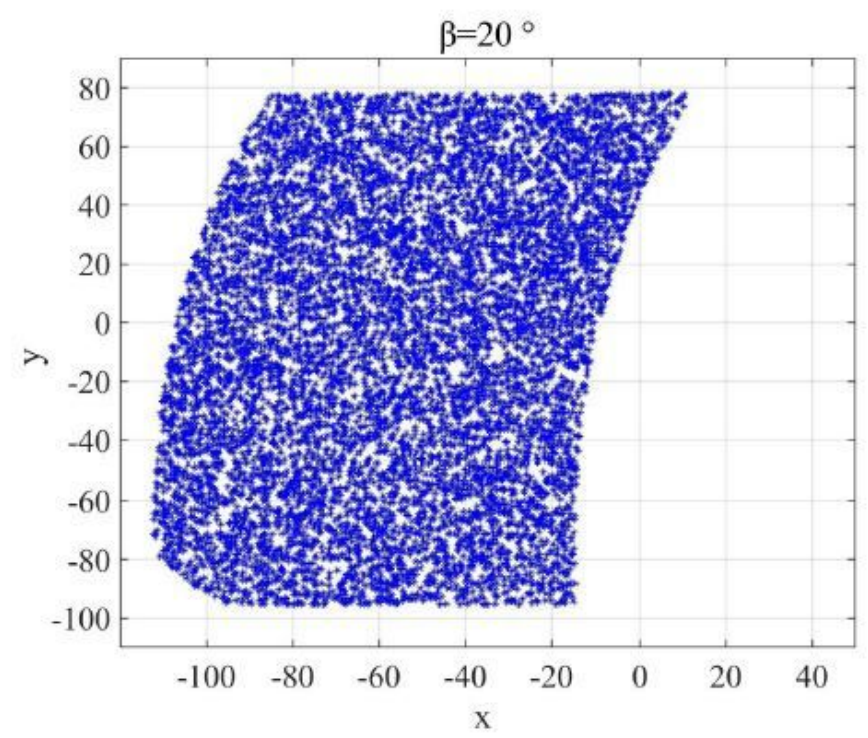

(d) $\beta=20^{\circ}$

Figure 19

Fixed posture workspace of $\beta$ 


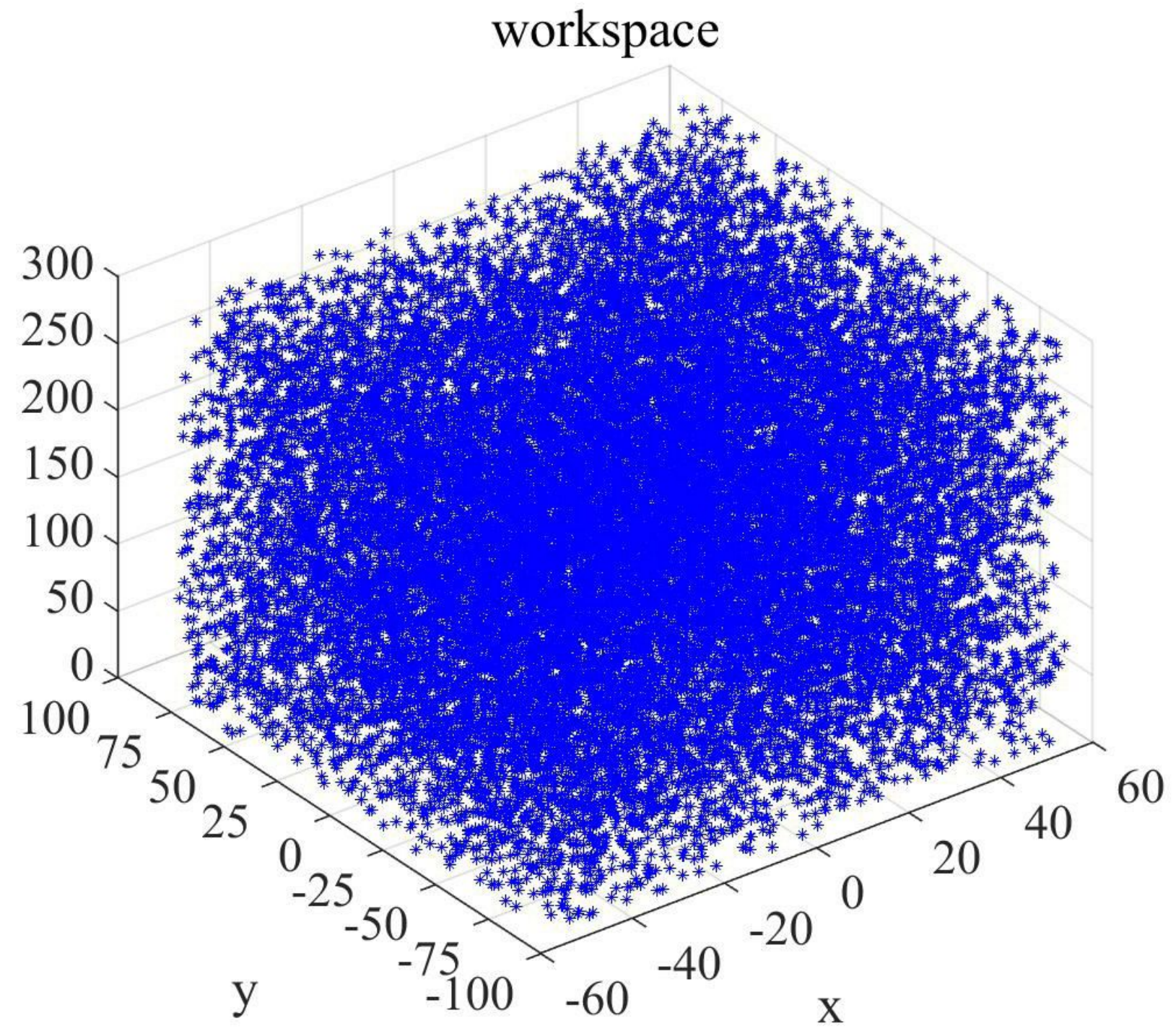

Figure 20

Fixed posture workspace 


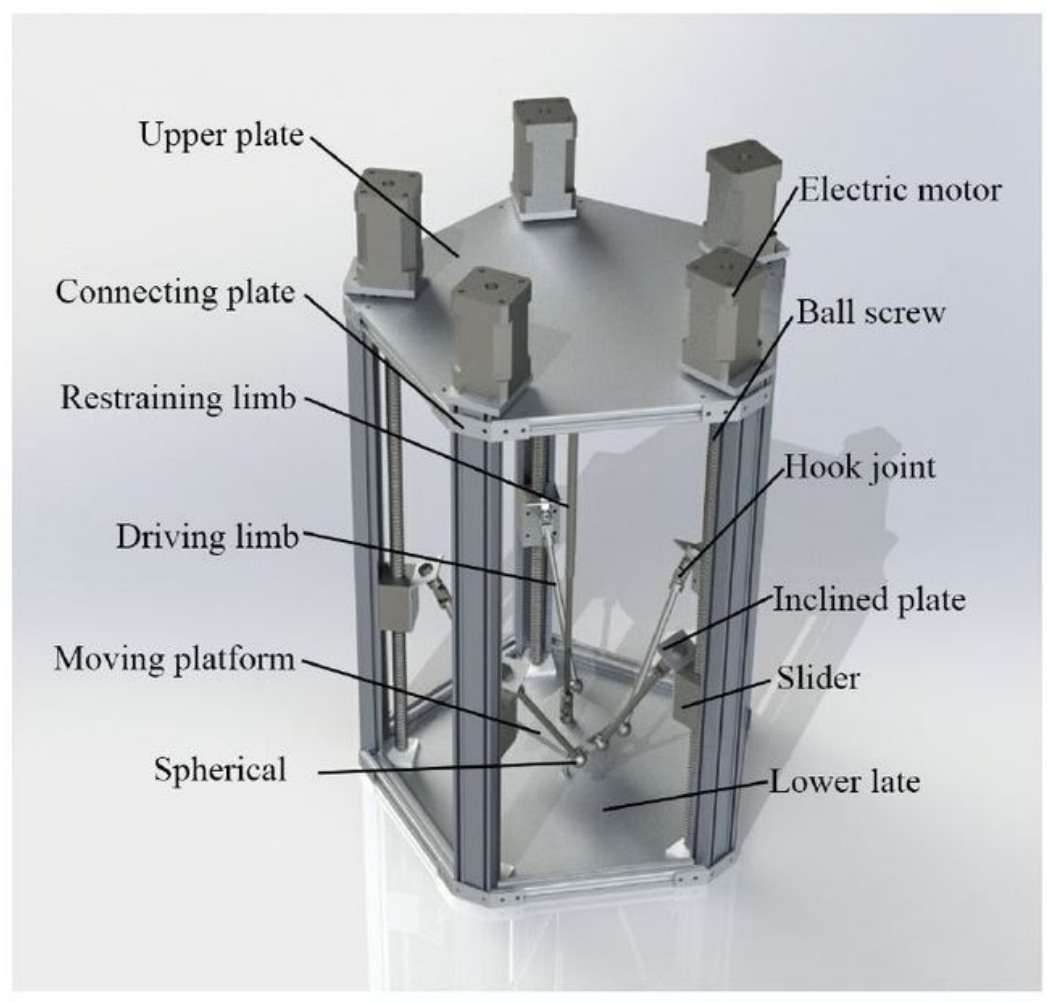

(a) 3D model of prototype

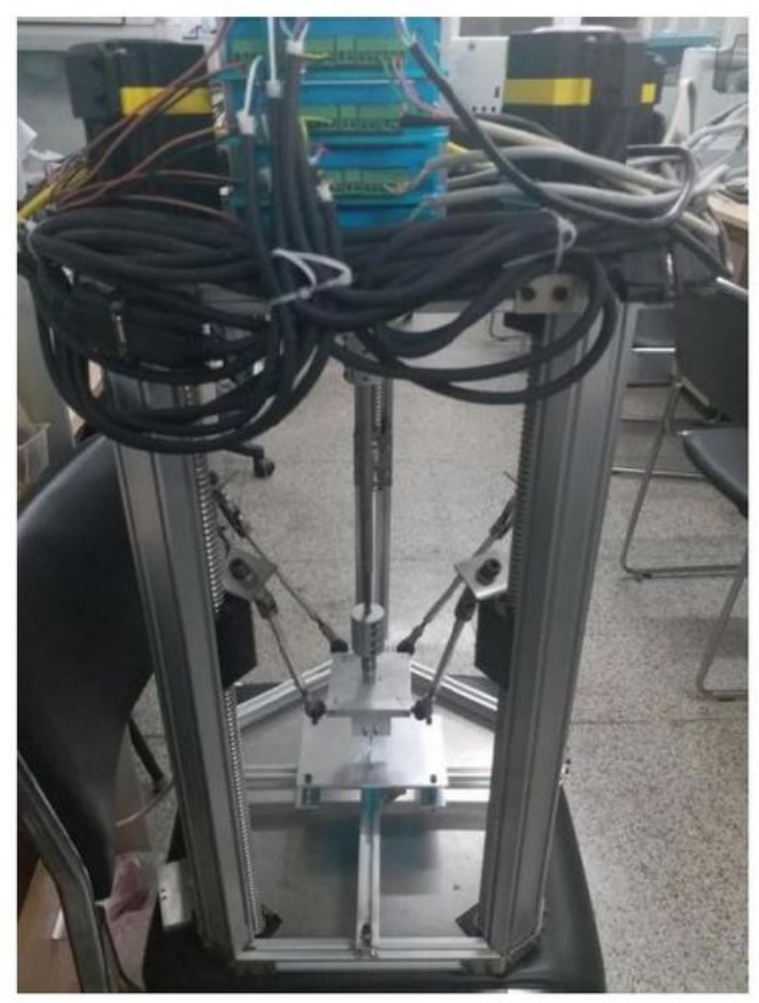

(b) Physical model of prototype

\section{Figure 21}

Prototype of parallel mechanism
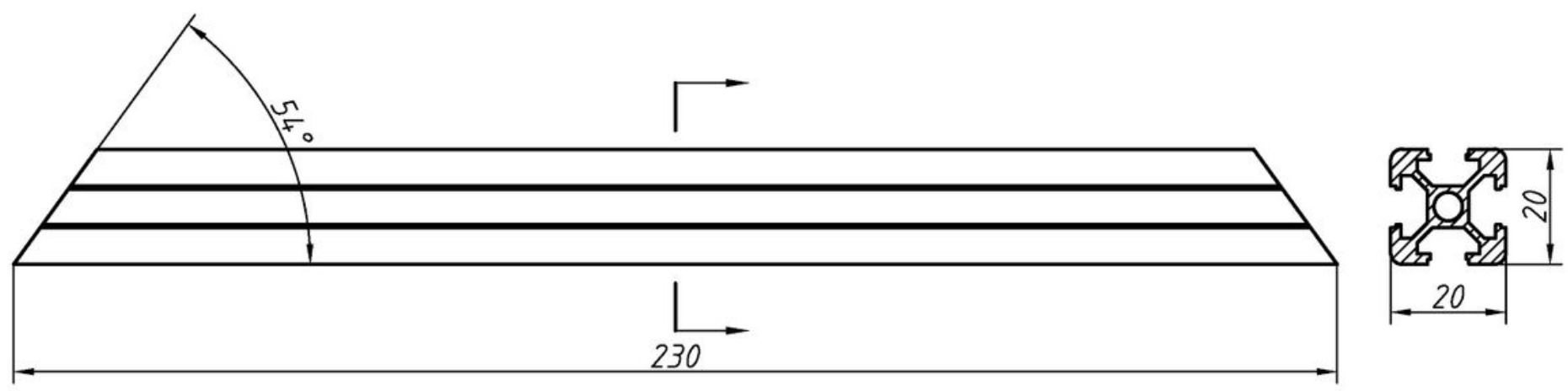

Figure 22

The size of profile 


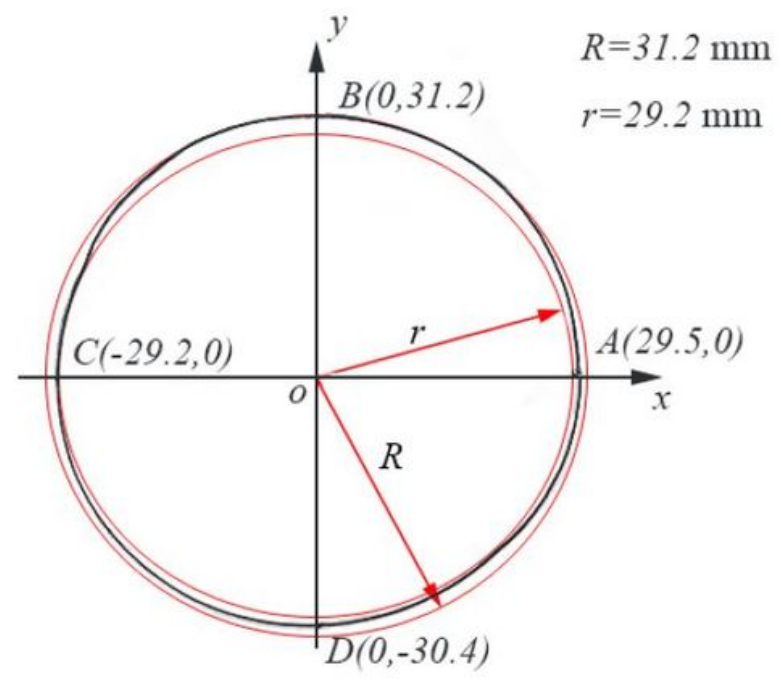

(a) $\alpha=0^{\circ}, \beta=0^{\circ}$

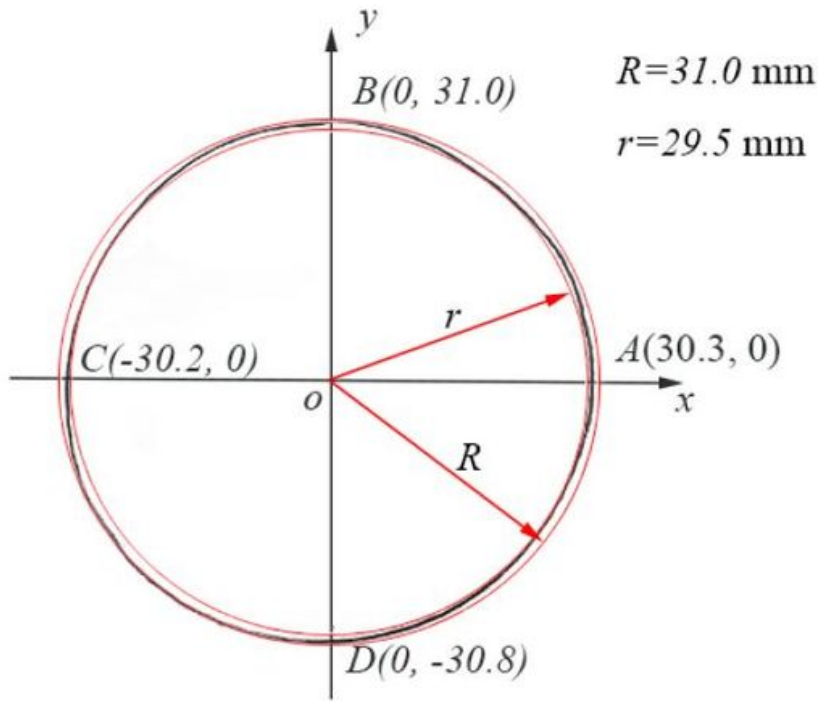

(b) $\alpha=0^{\circ}, \beta=-5^{\circ}$

Figure 23

Circular trajectories 